\title{
A Bimodal Burst Energy Distribution of a Repeating Fast Radio Burst Source
}

\section{Di Li ( $\nabla$ dili@nao.cas.cn )}

CAS Key Laboratory of FAST, NAOC, Chinese Academy of Sciences https://orcid.org/0000-0003-30107661

\section{P. Wang}

National Astronomical Observatories, Chinese Academy of Sciences

\section{Weiwei Zhu}

Chinese Academy of Sciences

\section{Bing Zhang}

University of Nevada, Las Vegas https://orcid.org/0000-0002-9725-2524

\section{Xinxin Zhang}

CAS Key Laboratory of FAST, NAOC, Chinese Academy of Sciences

\section{Ran Duan}

CAS Key Laboratory of FAST, NAOC, Chinese Academy of Sciences

\section{Yongkun Zhang}

CAS Key Laboratory of FAST, NAOC, Chinese Academy of Sciences

\section{Yi Feng}

CAS Key Laboratory of FAST, NAOC, Chinese Academy of Sciences

\section{Ningyu Tang}

CAS Key Laboratory of FAST, NAOC, Chinese Academy of Sciences

\section{Shami Chatterjee}

Cornell University https://orcid.org/0000-0002-2878-1502

\section{James Cordes}

Cornell University https://orcid.org/0000-0002-4049-1882

\section{Marylin Cruces}

Max-Planck-Institut für Radioastronomie

\section{Vishal Gajjar}

UC Berkeley

\section{George Hobbs}

CSIRO

\section{Chengjin Jin}

CAS Key Laboratory of FAST, NAOC, Chinese Academy of Sciences

\section{Michael Kramer}

Max-Planck-Institut für Radioastronomie 


\section{Duncan Lorimer}

West Virginia University https://orcid.org/0000-0003-1301-966X

\section{C.C. Miao}

National Astronomical Observatories, Chinese Academy of Sciences

\section{Chenhui Niu}

National Astronomical Observatories, Chinese Academy of Sciences

\section{J.R. Niu}

National Astronomical Observatories, Chinese Academy of Sciences

\section{Zhichen Pan}

National Astronomical Observatories, Chinese Academy of Sciences

\section{Lei Qian}

National Astronomical Observatories, Chinese Academy of Sciences https://orcid.org/0000-00030597-0957

\section{Shi Dai}

CSIRO Astronomy and Space Science

\section{Laura Spitler}

Max-Planck-Institute for Radio Astronomy

\section{Dan Werthimer}

University of California Berkeley

\section{Xiaoyao Xie}

Guizhou Normal University

\section{Youling Yue}

National Astronomical Observatories, Chinese Academy of Sciences

\section{Lei Zhang}

University of Chinese Academy of Sciences, https://orcid.org/0000-0001-8539-4237

\section{Qijun Zhi}

Guizhou Normal University

\section{Yan Zhu}

National Astronomical Observatories, Chinese Academy of Sciences

\section{Physical Sciences - Article}

Keywords: Bimodal burst energy distribution, repeating fast radio burst source, FRBs

Posted Date: October 20th, 2020

DOI: https://doi.org/10.21203/rs.3.rs-93082/v1

License: (9) This work is licensed under a Creative Commons Attribution 4.0 International License. Read Full License 
Version of Record: A version of this preprint was published at Nature on October 13th, 2021. See the published version at https://doi.org/10.1038/s41586-021-03878-5. 


\section{A bimodal burst energy distribution of a repeating fast radio burst source}

3

5 ${ }_{6}$ Werthimer $^{7}$, X. Y. Xie ${ }^{10}$, Y. L. Yue ${ }^{1}$, L. Zhang ${ }^{2,1,4}$, Q. J. Zhi ${ }^{10}$, Y. Zhu ${ }^{1}$

$7{ }^{1}$ CAS Key Laboratory of FAST, NAOC, Chinese Academy of Sciences, Beijing 100101, China

${ }_{8}^{2}$ University of Chinese Academy of Sciences, Beijing 100049, China

${ }_{9}^{3}$ Department of Physics and Astronomy, University of Nevada, Las Vegas, Las Vegas, NV 89154,

D. Li ${ }^{1,2 *}$ P. Wang ${ }^{1}$, W. W. Zhu ${ }^{1}$, B. Zhang ${ }^{3 \dagger}$ X. X. Zhang ${ }^{1}$, R. Duan ${ }^{1}$, Y. K. Zhang ${ }^{2,1}$, Y. Feng ${ }^{2,1,4}$, N. Y. Tang ${ }^{1}$, S. Chatterjee ${ }^{5}$, J. M. Cordes ${ }^{5}$, M. Cruces ${ }^{6}$, V. Gajjar ${ }^{7}$, G. Hobbs ${ }^{3}$, C. Jin ${ }^{1}$, M. Kramer ${ }^{6}$, D. R. Lorimer ${ }^{8,9}$, C. C. Miao ${ }^{1}$, C. H. Niu ${ }^{1}$, J. R. Niu ${ }^{1}$, Z. C. $\operatorname{Pan}^{1}$, L. Qian ${ }^{1}$, S. Dai ${ }^{4}$, L. Spitler ${ }^{6}$, D. USA

${ }^{4}$ CSIRO Astronomy and Space Science, PO Box 76, Epping, NSW 1710, Australia

${ }^{5}$ Cornell Center for Astrophysics and Planetary Science and Department of Astronomy, Cornell University, Ithaca, NY 14853, USA

${ }^{6}$ Max-Planck-Institut für Radioastronomie, Auf dem Hügel 69, D-53121 Bonn, Germany

${ }^{7}$ Department of Astronomy, University of California Berkeley, Berkeley, CA 94720

${ }^{8}$ Department of Physics and Astronomy, West Virginia University, P.O. Box 6315, Morgantown, WV 26506, USA

${ }^{9}$ Center for Gravitational Waves and Cosmology, West Virginia University, Chestnut Ridge Research Building, Morgantown, WV, USA

${ }^{10}$ Guizhou Normal University, Guiyang 550001, China

*Email:dili@nao.cas.cn orcid.org/0000-0003-3010-7661

†Email: zhang @ physics.unlv.edu orcid.org/0000-0002-9725-2524 
Fast radio bursts (FRBs) are cosmic sources that emit millisecond-duration radio pulses with a wide range of luminosities and yet unknown origin(s) ${ }^{\sqrt[1 / 2]{2}}$ A subset of FRBs were found to repeat, the prototype of which is the first precisely-located FRB $121102^{3}$, residing in a dwarf galaxy at redshift $z=0.193^{45^{5}}$. The source has been observed by most major telescopes and shows non-Poisson clustering of bursts over time, the hitherto highest burst rate, and a burst isotropic equivalent energy largely consistent with a power-law ${ }^{6-8}$, all of which are crucial characteristics to be compared to non-repeating sources. However, due to sensitivity limits, no true energy distribution of any FRB is known. Here we report the detection of 1652 independent bursts, more than quadruple the total of all previously published ones combined, in a total of 59.5 observing hours spanning 47 days using the Five-hundred-meter Aperture Spherical radio Telescope (FAST). The peak burst rate of $122 \mathrm{hr}^{-1}$ is by far the highest ever observed of any FRB. A characteristic peak in the isotropic equivalent energy distribution is found to be $\sim 4.8 \times 10^{37} \mathrm{erg}$ at $1.25 \mathrm{GHz}$, suggesting a possible threshold for producing abundant coherent radio bursts from FRBs. The burst energy distribution is optimally described by a bimodal distribution consisting of a log-normal function plus a Cauchy function. While no periodicity was found between $1 \mathrm{~ms}$ and $1000 \mathrm{~s}$, and the majority of the burst arrival times are consistent with being random, there exists a visible peak in the waiting time distribution at about $3.4 \mathrm{~ms}$, corresponding to significant clustering. Our results start to reveal the stochastic nature of abundant weaker bursts, which could be present in other FRB sources, apparently repeating or not. FRB generation mechanisms must be efficient and economical. Expensive triggers and/or contrived conditions for burst production seem unlikely. 
We have been carrying out a continuous monitoring campaign of FRB 121102 with FAST ${ }^{9}$ since August 2019. Between August 29 and October 29, 2019, we detected 1652 independent burst events (see Extended Table 1). The total number of previously published bursts from this source was 347 (Ref. ${ }^{6}+8$ and http://www.frbcat.org). The flux limit of this burst sample is at least three times lower than those of previous observations. The cadence and depth of the observations allow for a statistical study of the repeating bursts, revealing several previously unseen characteristics of FRB 121102. Fig. 1 depicts the burst statistics as a function of time, with the accumulated counts in one-hour bins in the upper panel and the day-to-day average burst rates in the lower panel. The burst rate separately peaked at $122 \mathrm{hr}^{-1}$ on September 7 th and $117 \mathrm{hr}^{-1}$ on October 1st, 2019. In both instances, the burst rate dropped precipitously afterwards.

We measured the peak flux density, pulse width, and fluence for each burst. Given the redshift $z=0.193^{5}$, we adopted the corresponding luminosity distance $D_{\mathrm{L}}=949 \mathrm{Mpc}$ based on the latest cosmological parameters measured by the Planck team ${ }^{10}$ and calculated the isotropic equivalent energy of each burst at $1.25 \mathrm{GHz}$ (see Methods). The derived energies span more than three orders of magnitude, from below $10^{37} \mathrm{erg}$ to near $10^{40} \mathrm{erg}$. Fig. 2 presents the histogram of the bursts (lower panel) and the cumulative counts as a function of energy (upper panel). With a prominent peak and two broad bumps, the distribution cannot be fit by a single power-law or a single lognormal function (Table 1). A satisfactory fit can be achieved with a log-normal distribution plus a Cauchy function:

$$
N(E)=\frac{N_{0}}{\sqrt{2 \pi} \sigma_{E} E} \exp \left[\frac{-\left(\log E-\log E_{0}\right)^{2}}{2 \sigma_{E}^{2}}\right]+\frac{\epsilon_{E}}{1+\left(\frac{E}{E_{0}}\right)^{\alpha_{E}}},
$$

where $\epsilon_{E}=0$ for $E<10^{38}$ erg and $\epsilon_{E}=1$ for $E>10^{38}$. The characteristic energy $E_{0}$ is determined 


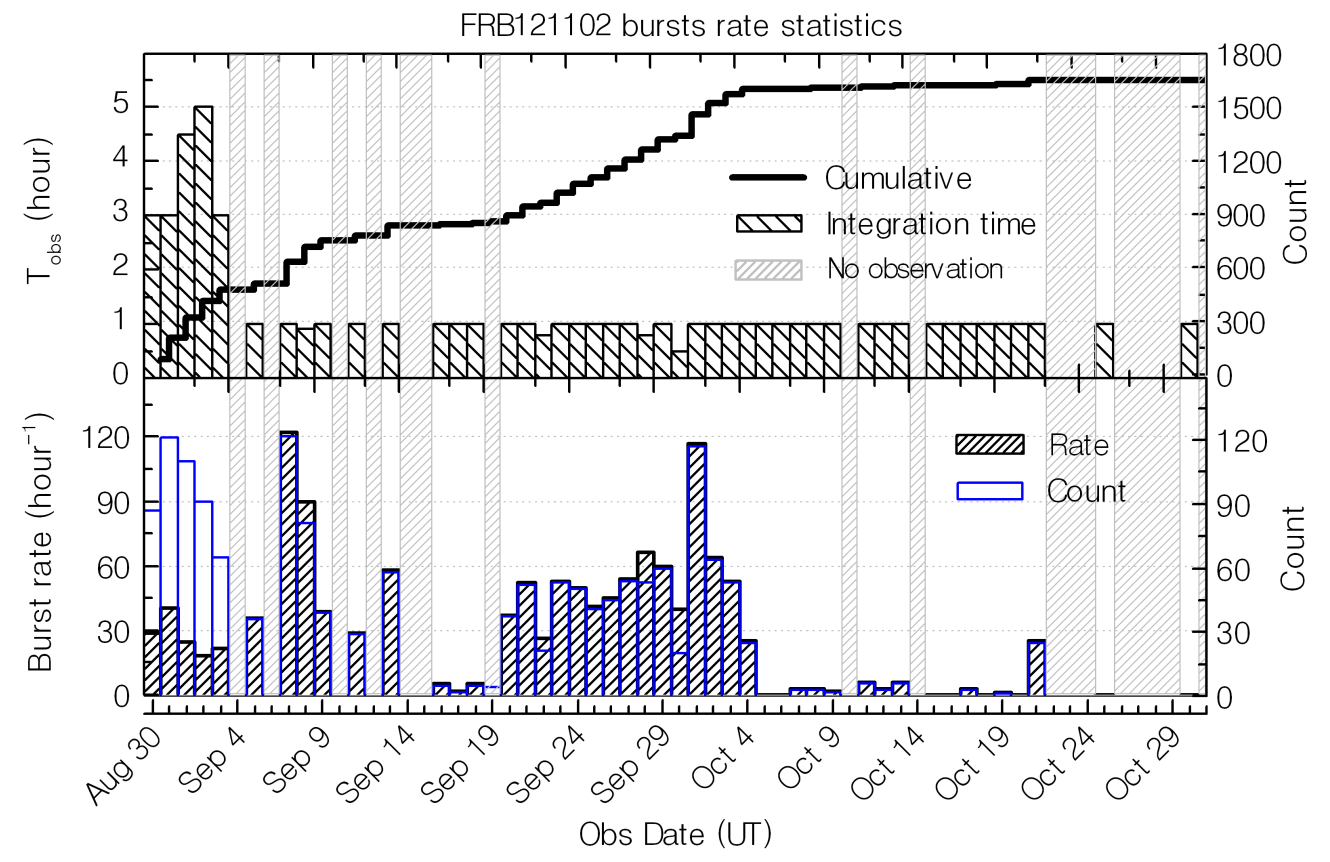

Figure 1: The detected bursts and their distribution during the observing campaign. Upper panel: observing hours and accumulated burst number. Lower panel: burst counts and burst rate. The grey shaded bars denote days without observations of the source.

No periodicity between $1 \mathrm{~ms}$ to $1000 \mathrm{~s}$ could be found in the power spectrum calculated 
(UT) $2019 / 08 / 29-2019 / 10 / 29$

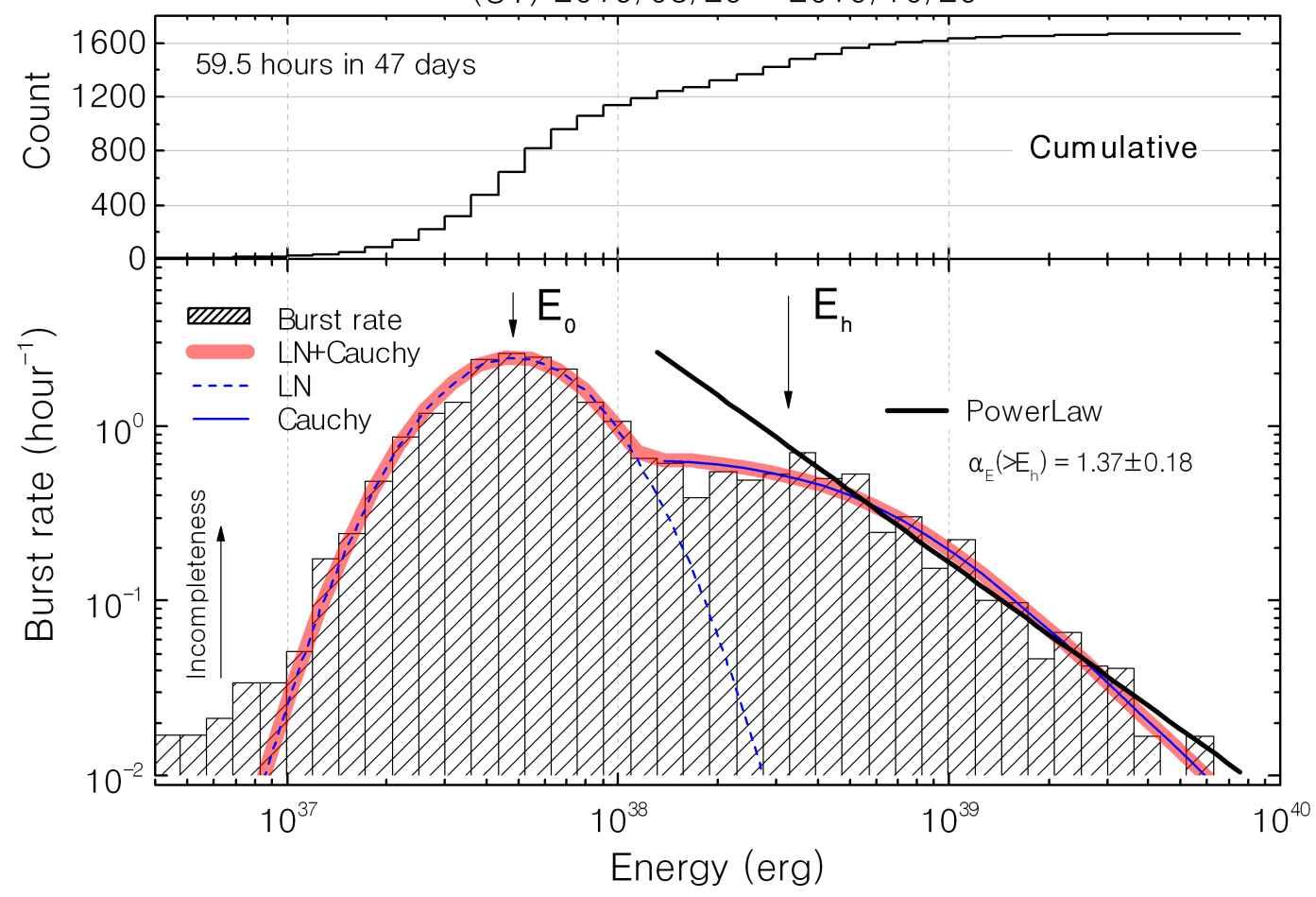

Figure 2: The burst rate distribution of the isotropic equivalent energy at $1.25 \mathrm{GHz}$ for FRB

121102 bursts. The bimodal 'lognormal(LN-dashed blue) + Cauchy (solid blue)' distribution is shown in red and a single power-law fit for bursts above a certain threshold $E \geq E_{h}=3 \times 10^{38} \mathrm{erg}$ is shown in black. Judging by experience, the weak bursts with peak flux smaller $\sim 9 \sigma$ cannot be recovered completely (see Methods), as indicated by the upward arrow.

using either the FFT or the Lomb-Scargle Periodogram (see Methods). The waiting time between two adjacent (detected) bursts is $\delta \mathrm{t}=t_{\mathrm{i}+1}-t_{\mathrm{i}}$, where $t_{\mathrm{i}+1}$ and $t_{\mathrm{i}}$ are the arrival times for the $(i+1) \mathrm{th}$ and (i)th bursts, respectively. Each pulse time of arrival (ToA) was transformed to the solar system barycentre using the DE405 ephemeris. 
All waiting times were calculated for pulses within the same session to avoid long gaps of $\sim 24 \mathrm{~h}$. The distribution of the waiting times (Fig. 3) has a dominant feature that can be well fit by a log-normal function centered at $70 \pm 12$ s. Selecting only high energy pulses $E>3 \times 10^{38} \mathrm{erg}$, the peak moves to 220 seconds. There is also a secondary peak centered at $\sim 3.4 \mathrm{~ms}$ that is related to burst substructure like that seen in Fig. 11 or by closely-spaced, independent bursts. All behaviours described above are generally consistent with previous findings for FRB $121102^{7||^{\mid 12} \mid 13}$ and can be reproduced within the uncertainties by simulating random bursts. For example, the location of the peak of the log-normal distribution can be obtained with a Monte-Carlo simulation mimicking the sampling cadence and number of detections of the real observations (see Methods).

Such a waiting time distribution can also be accounted for by external modulating influences, e.g. a rotating object with a vast range of emission altitudes. These stochastic delays can erase any signature of the underlying periodicity in the power spectrum or the waiting-time distribution, while the 70s waiting-time peak still places an upper bound on the underlying period ${ }^{114}$.

The optimal dispersion measure (DM) value of the bursts is constrained to $565.8 \pm 0.9 \mathrm{pc} \mathrm{cm}^{-3}$ between MJD 58,724 and MJD 58,776 (see Methods). This suggests that the DM of FRB 121102 has increased by $\sim 5-8 \mathrm{pc} \mathrm{cm}^{-3}$ (or $\sim 1-1.4 \%$ ) compared to earlier detections $\frac{15]}{16}$, confirming a trend seen before with larger significance (Fig (4). Combining all the data, one can derive

$$
\frac{d \mathrm{DM}}{d t}=+0.85 \pm 0.10 \mathrm{pc} \mathrm{cm}^{-3} \mathrm{yr}^{-1} \text {. }
$$

This is inconsistent with the decreasing-trend predicted for a freely expanding shell (e.g. a supernova remnant) around the FRB source ${ }^{17}$, but is consistent with such a shell during the deceleration 


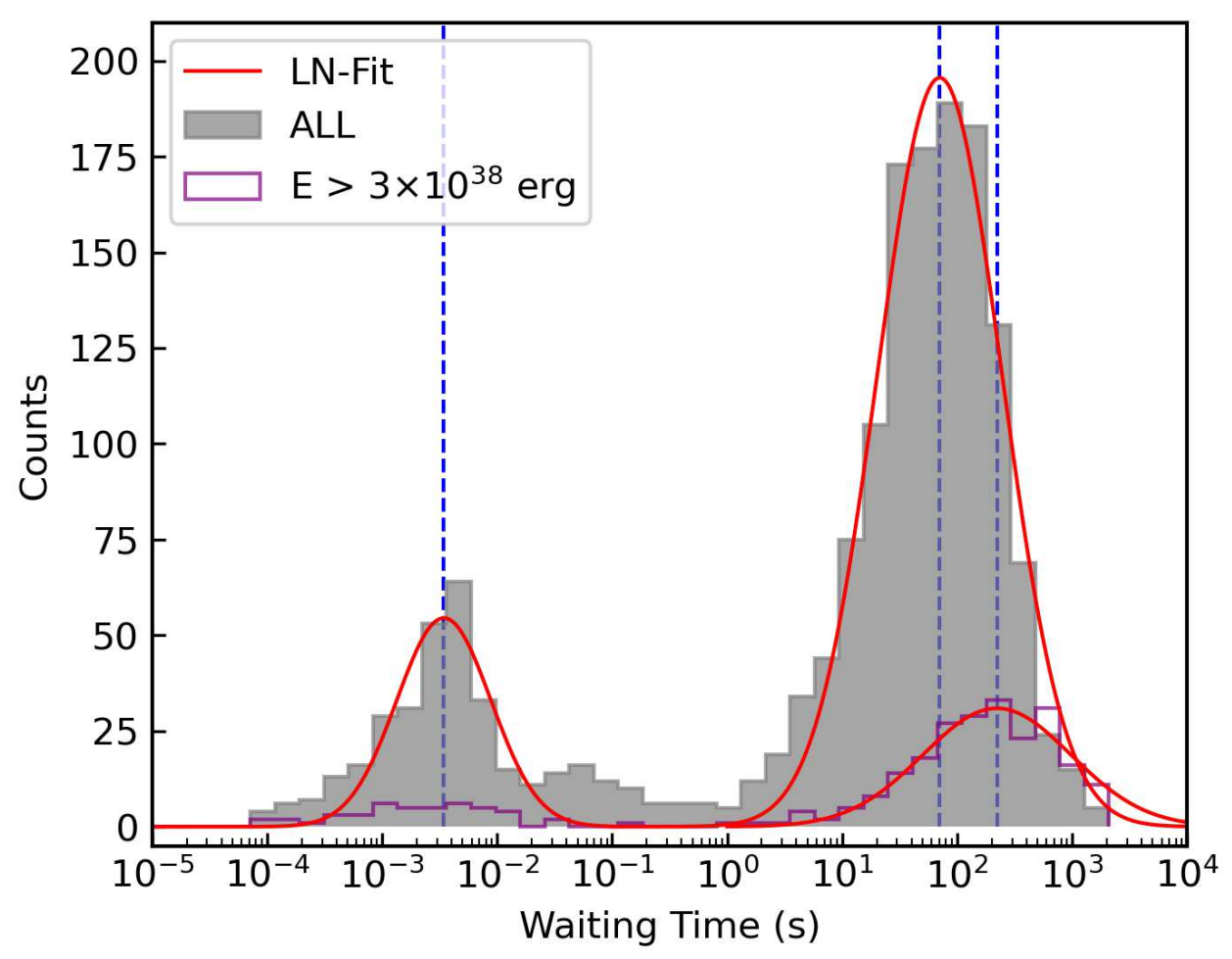

Figure 3: The waiting time distribution of the bursts. The grey bar and solid red curve denote the distribution of waiting time and its log-normal (LN) fit. The high energy component is shown in solid purple line $\left(E>3 \times 10^{38} \mathrm{erg}\right)$. The three fitted peak waiting times (blue dashed vertical lines) from left to right are $3.4 \pm 1.0 \mathrm{~ms}, 70 \pm 12 \mathrm{~s}$, and $220 \pm 100 \mathrm{~s}$, respectively.

(Sedov-Taylor) phase ${ }^{18 / 19}$.

The large sample of bursts sheds new lights on theoretical models of FRBs. The isotropic equivalent energy distribution (or energy function) necessitates a bimodal fit, suggesting possibly more than one emission mechanism or emission site/beam-shape, even for this one source. The log-normal distribution reflects the stochastic nature of the weak bursts, the generation of which seem to become less efficient below the characteristic energy scale of $E_{0} \sim 4.8 \times 10^{37}$ ergs. Some 

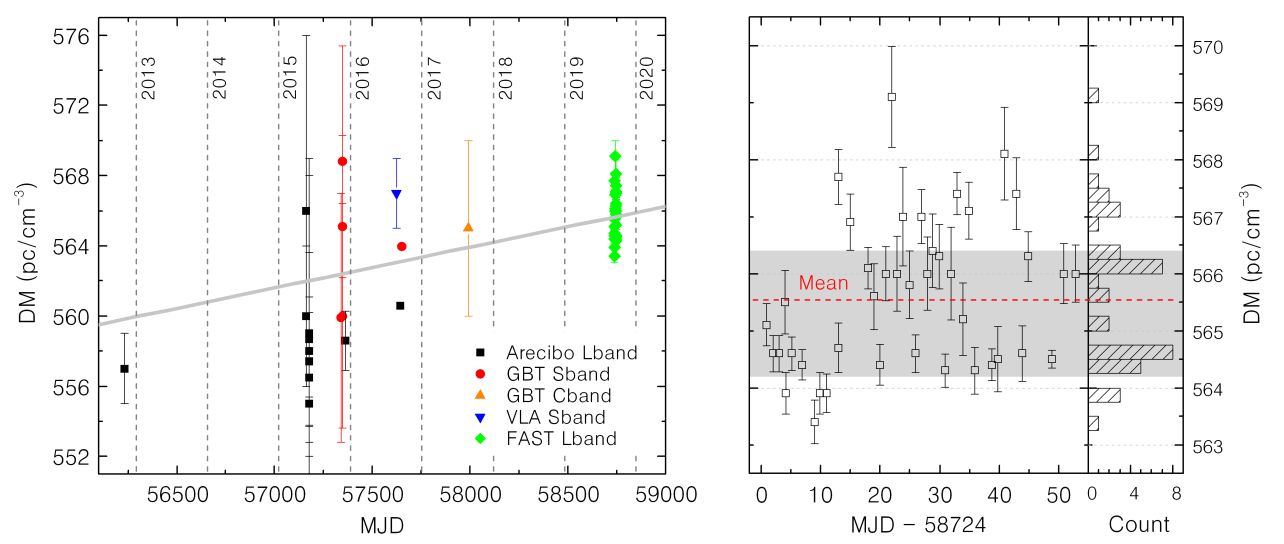

Figure 4: The DM evolution of FRB 121102. Left panel: Temporal DM variation for FRB 121102 over the years. The solid grey line denotes the corresponding linear fitting with a growth rate $+0.85 \pm 0.10 \mathrm{pc} \mathrm{cm}^{-3} \mathrm{yr}^{-1}$. Right panel: the distribution of optimum DM for one of brightest burst in each day during the FAST observations. All the bursts are considered to have a conservative statistical error of FWHM for squared derivative profiles with each DM trial. The red dashed line indicates averaged DM $565.6 \mathrm{pc} \mathrm{cm}^{-3}$ during the FAST observation, and the grey region shows the 95\% confidence level.

magnetar models do predict a luminosity lower bound for producing $\mathrm{FRBs}^{2021}$, but the predicted values $\left(\sim 7.5 \times 10^{37}-10^{40} \mathrm{erg}\right)$ are generally larger than the $E_{0}$ value reported here. The Cauchy function can describe the ratio between two normally distributed random variables, thus could suggest correlated events for generating strong bursts.

As shown in Fig. 3, the distribution of the time intervals between bursts (henceforth referred to as the waiting times) is log-normal in form. This behaviour is similar to that observed in other astrophysical bursting events such as soft gamma-ray repeaters (SGRs) 2223 . The extremely-high 
burst rate revealed by our observational campaign poses challenges to some models. For example, for the model invoking asteroids colliding a neutron star to produce $\mathrm{FRBs}^{24}$, the excessive number of bursts worsens the requirement for the already demanding asteroid belt number density 25 . The FRB triggering mechanism has to be economical. Models invoking giant-pulse-like emission ${ }^{26}$, active magnetar emission ${ }^{20127}$, or persistent magnetosphere interactions ${ }^{\sqrt{28}}$ are attractive options.

The frequent triggers of bursts also constrain coherent radiation models. In particular, synchrotron maser models that can produce near-100\% polarization demand well-ordered magnetic field lines and extremely high radiation efficiencies ${ }^{2930}$. Both requirements are challenged by the data. The short waiting time makes it difficult for the emission region to restore ordered magnetic field configuration, and the low efficiency makes the bursts expensive, further aggravating the burst triggering problem. Conversely, coherent emission mechanisms that invoke a neutron star magnetosphere ${ }^{\frac{\sqrt{31 / 32}}{1}}$ can efficiently radiate in the radio band, and are preferred by the data.

1. Petroff, E., Hessels, J. W. T. \& Lorimer, D. R. Fast radio bursts. A\&A Rev. 27, 4 (2019).

2. Cordes, J.M., Chatterjee, S. \& Fast Radio Bursts: An Extragalactic Enigma. ARA\&A 57, 417-465 (2019).

3. Spitler, L. G. et al. A repeating fast radio burst. Nature 531, 202-205 (2016).

4. Chatterjee, S. et al. A direct localization of a fast radio burst and its host. Nature 541, 58-61 (2017). 
5. Tendulkar, S. P. et al. The Host Galaxy and Redshift of the Repeating Fast Radio Burst FRB 121102. ApJL 834, L7 (2017).

6. Law, C. J. et al. A Multi-telescope Campaign on FRB 121102: Implications for the FRB Population. ApJ 850, 76 (2017).

7. Zhang, Y. G. et al. Fast Radio Burst 121102 Pulse Detection and Periodicity: A Machine Learning Approach. ApJ 866, 149 (2018).

8. Gourdji, K. et al. A Sample of Low-energy Bursts from FRB 121102. ApJL 877, L19 (2019).

9. Li, Di. et al. FAST in Space: Considerations for a Multibeam, Multipurpose Survey Using China’s 500-m Aperture Spherical Radio Telescope (FAST). IEEE 19, 112-119 (2018).

10. Planck Collaboration et al. Planck 2015 results. XIII. Cosmological parameters. A\&A 594, A13 (2016).

11. Shannon, R. M. et al. The dispersion-brightness relation for fast radio bursts from a wide-field survey. Nature 562, 386-390 (2018).

12. Katz, J. I. Fast radio bursts. Progress in Particle and Nuclear Physics 103, 1-18 (2018).

13. Palaniswamy, D., Li, Y. \& Zhang, B. Are There Multiple Populations of Fast Radio Bursts? ApJL 854, L12 (2018).

14. Cordes, J.M., Wasserman, I. Batra, G. Chatterjee, S. \& Aperiodic and Periodic Radio Bursts from Young Precessing Magnetars. in prep. 
15. Scholz, P. et al. The Repeating Fast Radio Burst FRB 121102: Multi-wavelength Observations and Additional Bursts. ApJ 833, 177 (2016).

16. Petroff, E. et al. FRBCAT: The Fast Radio Burst Catalogue. PASA 33, e045 (2016).

17. Metzger, B. D., Berger, E. \& Margalit, B. Millisecond Magnetar Birth Connects FRB 121102 to Superluminous Supernovae and Long-duration Gamma-Ray Bursts. ApJ 841, 14 (2017).

18. Yang, Y.-P. \& Zhang, B. Dispersion Measure Variation of Repeating Fast Radio Burst Sources. ApJ 847, 22 (2017).

19. Piro, A. L. \& Gaensler, B. M. The Dispersion and Rotation Measure of Supernova Remnants and Magnetized Stellar Winds: Application to Fast Radio Bursts. ApJ 861, 150 (2018).

20. Wadiasingh, Z. et al. The Fast Radio Burst Luminosity Function and Death Line in the Lowtwist Magnetar Model. ApJ 891, 82 (2020).

21. Kumar, P. \& Bosnjak, Z. FRB Coherent Emission from Decay of Alfven Waves. arXiv e-prints arXiv:2004.00644 (2020). 2004.00644.

22. Göğüs, E. et al. Statistical Properties of SGR 1806-20 Bursts. ApJL 532, L121-L124 (2000).

23. Wang, F. Y. \& Yu, H. SGR-like behaviour of the repeating FRB 121102. JCAP 2017, 023 (2017).

24. Dai, Z. G., Wang, J. S., Wu, X. F. \& Huang, Y. F. Repeating Fast Radio Bursts from Highly Magnetized Pulsars Traveling through Asteroid Belts. ApJ 829, 27 (2016). 
25. Smallwood, J. L., Martin, R. G. \& Zhang, B. Investigation of the asteroid-neutron star collision model for the repeating fast radio bursts. MNRAS 485, 1367-1376 (2019).

26. Cordes, J. M. \& Wasserman, I. Supergiant pulses from extragalactic neutron stars. MNRAS 457, 232-257 (2016).

27. Wang, W. et al. FRB 121102: A Starquake-induced Repeater? ApJ 852, 140 (2018).

28. Zhang, B. Fast Radio Bursts from Interacting Binary Neutron Star Systems. ApJL 890, L24 (2020).

29. Metzger, B. D., Margalit, B. \& Sironi, L. Fast radio bursts as synchrotron maser emission from decelerating relativistic blast waves. MNRAS 485, 4091-4106 (2019).

30. Beloborodov, A. M. Blast Waves from Magnetar Flares and Fast Radio Bursts. arXiv e-prints (2019).

31. Kumar, P., Lu, W. \& Bhattacharya, M. Fast radio burst source properties and curvature radiation model. MNRAS 468, 2726-2739 (2017).

32. Yang, Y.-P. \& Zhang, B. Bunching Coherent Curvature Radiation in Three-dimensional Magnetic Field Geometry: Application to Pulsars and Fast Radio Bursts. ApJ 868, 31 (2018).

Acknowledgements This work is supported by National Natural Science Foundation of China (NSFC) Programs No. 11988101, No. 11725313, No. 11690024, No. U1731238; by CAS International Partnership Program No. 114-A11KYSB20160008; by CAS Strategic Priority Research Program No. XDB23000000; and the National Key R\&D Program of China (No. 2017YFA0402600). S.C. and J.M.C. acknowledge 
Competing Interests The authors declare that they have no competing financial interests.

support from the National Science Foundation (AAG 1815242). D.R.L. acknowledges support from support from the National Science Foundation awards AAG-1616042, OIA-1458952 and PHY-1430284. PW and CHN acknowledge support from cultivation project for FAST scientific payoff and research achievement of CAMS-CAS. This work made use of data from FAST, a Chinese national mega-science facility built and operated by the National Astronomical Observatories, Chinese Academy of Sciences.

Author Contributions DL, RD, WWZ launched the FAST campaign; PW, CHN, YKZ, YF, NYT, JRN, CCM, LZ processed the data; DL, BZ, PW drafted the paper; RD, XXZ, VG, CJ, YZ, DW, YLY built the FAST FRB backend; LQ, GH, XYX, QJZ, SD made key contributions to the overall FAST data processing pipelines; LS, MC, MK provided salient information on FRB 121102 from other observatories, particularly Effelsberg, and contributed to the scientific analysis; SC, JMC, DRL made numerous corrections to the writing and analysis. JMC, in particular, pointed out the errors in the noise floor analysis in the original draft. 


\section{Methods}

Burst energetics In order to obtain high quality flux density and polarization calibration solutions, a $1 \mathrm{~K}$ equivalent noise calibration signal was injected before each session, which was used to scale data to $T_{\text {sys }}$ units. The bottom panel of Fig 5 shows the off-pulse brightness (mK s) of the first pulse in each session. The standard deviation of off-pulse brightness is constant within $6 \%$ for all observations. The variation in the off-pulse level comes mainly from the zenith angle dependence of the telescope gain. Kelvin units were then converted to mJy using the zenith angle-dependent gain curve, provided by the observatory through quasar measurements. The upper panel of Fig 5 shows the Zenith-angle-dependent gain applied for each pulse. The red dots denote the average gain in each day. For most days, the pulses that have brightness closest to the average value were taken at zenith angles $<15$ degrees, which corresponds to a stable gain of $16 \mathrm{~K} / \mathrm{Jy}$. We searched the data with both Heimdall ${ }^{33}$ and PRESTO-single-pulse ${ }^{34}$ pipelines. The resulting candidates were selected with $\gtrsim 7 \sigma$ peak flux then manually inspected and cross-checked though pulse dynamicspectra. For pulses with peak flux less than $\lesssim 9 \sigma$, the verification of candidates were much affected by the radio frequency interference (RFI) environments and the characteristics of the individual pulses, particularly, its bandwidth in frequency (some as narrow as a few tens of MHz). We carried out a systematic search twice. The empirical results indicate a rather complete pulse sample for all bursts with $\gtrsim 10 \sigma$ peak flux, which is still well below the peak of the distribution of this sample. The incompleteness of the weak pulses, thus, only affect to a minor degree of the width of the following log-normal distribution, but not its peak location. 
We calculated isotropic equivalent burst energy, $E$ following Equation (9) of Ref. 35 :

$$
E=\left(10^{39} \mathrm{erg}\right) \frac{4 \pi}{1+z}\left(\frac{D_{L}}{10^{28} \mathrm{~cm}}\right)^{2}\left(\frac{F_{v}}{\mathrm{Jy} \cdot \mathrm{ms}}\right)\left(\frac{v_{c}}{\mathrm{GHz}}\right)
$$

where $F_{v}=S_{v} \times W_{\text {eq }}$ is the specific fluence in units of $\mathrm{erg} \mathrm{cm}^{-2} \mathrm{~Hz}^{-1}$ or Jy $\cdot \mathrm{ms}, S_{v}$ is the peak flux density which has been calibrated with the noise level of the baseline, and then measured the amount of pulsed flux above the baseline, giving the flux measurement for each pulses at a central frequency of $v_{c}=1.25 \mathrm{GHz}, W_{\text {eq }}$ is the equivalent burst duration, and the luminosity distance $D_{L}$ $=949 \mathrm{Mpc}$ corresponds to a redshift $z=0.193$ for FRB $121102^{5}$.

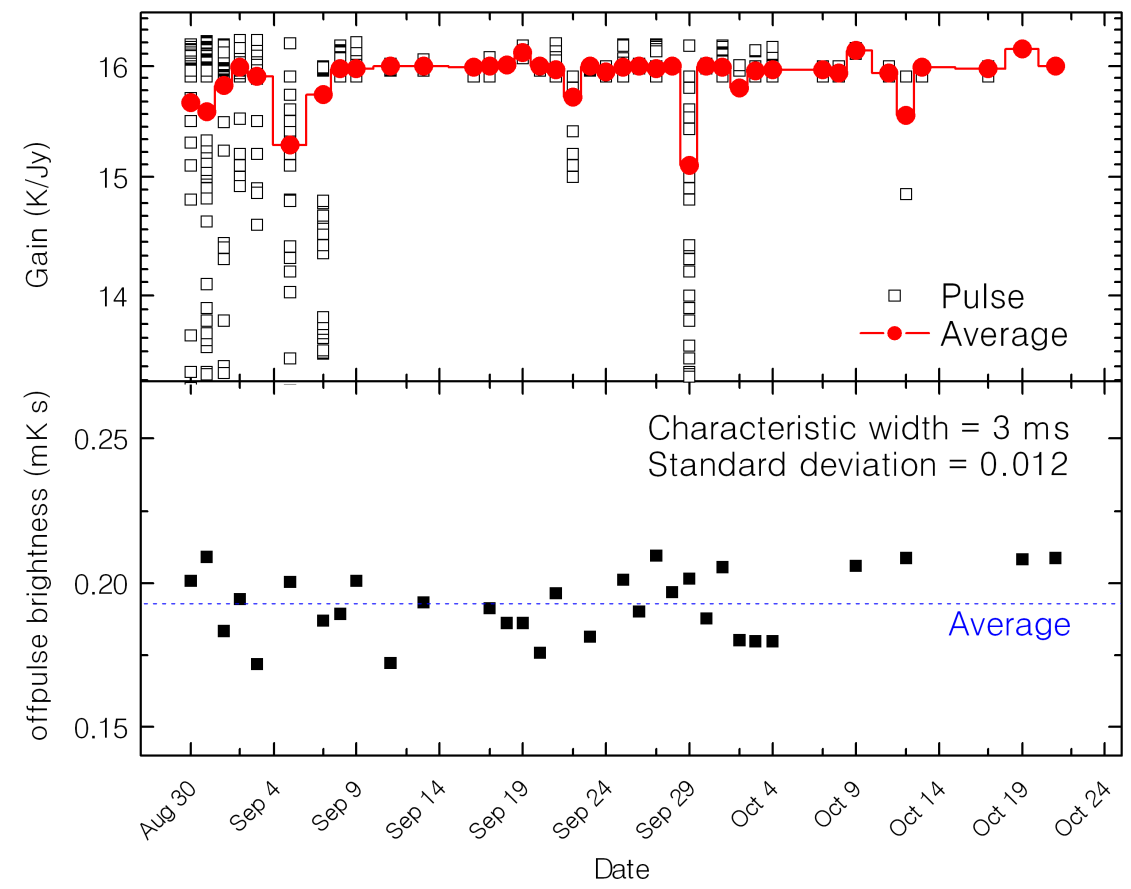

Figure 5: The distribution of the instrumental gain and off-pulse brightness RMS at 1.25 GHz

for observations. The upper panel indicates the gain applied for each pulses. The red dots denote the averaged gain in each day. The bottom panel shows the off-pulse brightness RMS (mK s) of first pulse in each day. 
Energy Distribution The histogram of burst energies exhibits two clearly separable bumps, which can be well fit by two log-normal function (Fig. 6).

The burst rate versus burst energy distribution shows a broad bump centered around $4.8 \times$ $10^{37} \mathrm{erg}$ and a power-law-like distribution with a slope close to -2 for the high energy tail. We first fit the distribution with a single power-law, Cauchy, and log-normal function, respectively. The $\chi^{2}$ and $\mathrm{R}^{2}$ tests in Table 1 show that these single-component models cannot adequately describe the data.

Table 1: The fitted parameters of the isotropic equivalent energy distribution.

\begin{tabular}{ccccc}
\hline \hline Function & Fitting parameter & Energy range (erg) & Reduced $\chi^{2} \dagger$ & $\mathrm{R}^{2} \dagger$ \\
\hline Power law & $\gamma=-0.61 \pm 0.04$ & $4 \times 10^{36} \leq E \leq 8 \times 10^{39}$ & $0.689(8)$ & $0.104(6)$ \\
& $\gamma=-1.37 \pm 0.18$ & $3 \times 10^{38} \leq E \leq 8 \times 10^{39}$ & $0.004(1)$ & $0.999(1)$ \\
\hline \multirow{3}{*}{ Lognormal } & $E_{0}=7.62 \times 10^{37}$ & & & \\
& $N_{0}=2.20 \times 10^{38}$ & $4 \times 10^{36} \leq E \leq 8 \times 10^{39}$ & $0.056(9)$ & $0.86(8)$ \\
& $\sigma_{E}=0.54$ & & & \\
\hline Cauchy & $E_{0}=8.16 \times 10^{38}$ & $4 \times 10^{36} \leq E \leq 8 \times 10^{39}$ & $0.438(1)$ & $0.113(1)$ \\
& $\alpha_{E}=3.02 \pm 0.5$ & & & \\
\hline \multirow{3}{*}{ Lognormal+Cauchy } & $E_{0}=7.2 \times 10^{37}$ & & & \\
& $N_{0}=2.06 \times 10^{38}$ & $4 \times 10^{36} \leq E \leq 8 \times 10^{39}$ & $0.037(4)$ & $0.931(7)$ \\
& $\sigma_{E}=0.52$ & & & \\
& $\alpha_{E}=1.85 \pm 0.3$ & & & \\
\hline
\end{tabular}

* Uncertainties in parentheses refer to the last quoted digit.

$\dagger$ Reduced $\chi^{2}$ is obtained by the best fitting method with 20 iterations.

$\ddagger$ Coefficient of determination, $R^{2}=1-S_{\text {res }} / S_{\text {tot }}$, where $S_{\text {tot }}$ is total sum of squares from data, and $S_{\text {res }}$ is the minimum fitting residual sum of squares. 
We then test the hypothesis that the energy distribution can be described by a power-law function $N(E)=N_{1} E^{-\alpha_{E}}$ in certain energy range $E_{i} \leq E \leq E_{f}$, i.e.

$$
N_{1}=\frac{N_{e v}\left(1-\alpha_{E}\right)}{E_{f}^{1-\alpha_{E}}-E_{i}^{1-\alpha_{E}}},
$$

where $N_{1}$ is normalization constant, and $N_{e v}$ is the total number of bursts included. For $3 \times 10^{38} \leq$ $E \leq 8 \times 10^{39} \mathrm{erg}$, the energy function is consistent with a power law with $\alpha_{E}=1.37 \pm 0.18$, consistent with the results obtained from the ASKAP sample and all the bursts from the FRB catalog $811,36-38$. A bimodal distribution is clearly needed to properly cover the full energy range. A log-normal function plus a Cauchy function for the high energy range can achieve a satisfactory fit, with a coefficient of determination $R^{2}=0.931$.

In Fig. 7, temporal variation were seen for the collective behaviours of each session. There are days with significantly brighter average, although weak bursts are always present.

Width distribution Fig. 8 shows pulse width $W_{\text {eq }}$ against flux density (left) and the pulse width distribution of the bursts (right). The equivalent width $W_{\text {eq }}$ is defined as the width of a rectangular burst that has the same area as the bursts, with the height of peak flux density denoted as $S_{\text {peak. }}$ In our sample, several pulses might be described as multiple components in a single burst, if there is "bridge" emission (higher than $5 \sigma$ ) between pulses for the bursts with a complex time-frequency structure. This results in some bursts being overestimated in equivalent width. The computed equivalent widths range from $0.43 \mathrm{~ms}$ to $\sim 40 \mathrm{~ms}$, consistent with a log-normal distribution centered around $\sim 4 \mathrm{~ms}$. This is consistent with the known statistical properties of repeating FRBs 39 . 


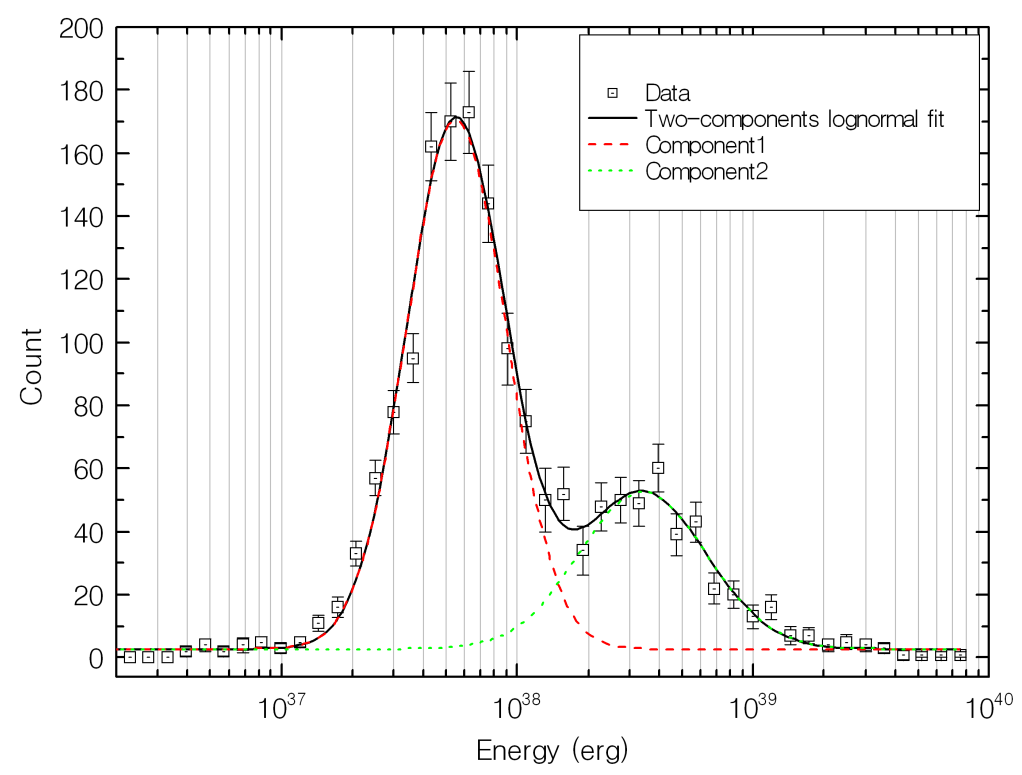

Figure 6: The distribution of the isotropic equivalent energy at $1.25 \mathrm{GHz}$ for FRB 121102 bursts. The two-component lognormal distribution is separately fitted in red and green dash lines, an overall fit for bursts is shown in black.

Monte-Carlo simulations of the waiting time distribution Following the exact setup of the observations, including starting time, duration, sampling rate, and pulse burst rate, we generate random ToAs through Monte Carlo simulations. The number of generated random bursts are 16000 times, 100 times, 1 time, and 0.2 times of the real ToAs, respectively. The distributions of different waiting time sets are shown in Fig. 9. The log-normal distribution appears in the randomly-generated waiting time distribution.

The peak waiting time of the log-normal distribution increases as the number of bursts in the simulated sample decreases. Among the 1652 pulses of FRB 121102, 296 have higher energy 


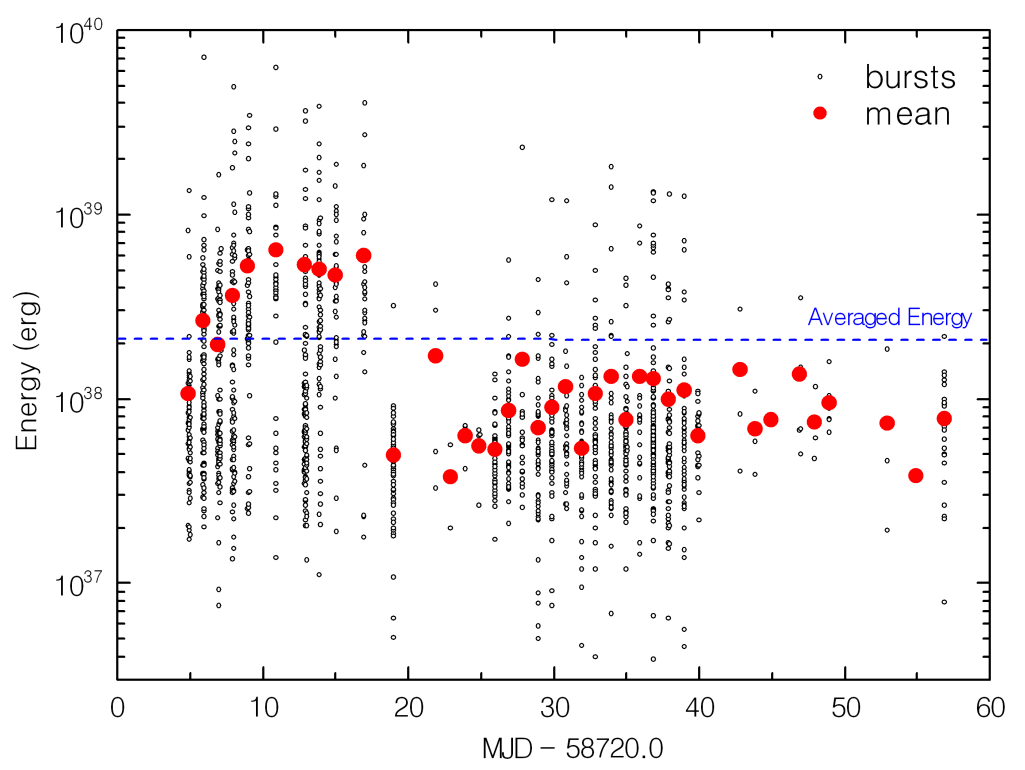

Figure 7: Energy of each burst. The red dots represent the average value for each observing session. An overall averaged is shown as a dashed blue line.

than $3 \times 10^{38} \mathrm{erg}$, which accounts for about one-fifth of the total pulses. The peak waiting time of the 1 time and 0.2 times simulated data are $63 \mathrm{~s}$ and $283 \mathrm{~s}$, which are close to the waiting time of real data, or within the margin of error. As for the peak around $3.4 \mathrm{~ms}$ in real data, if the waiting time of randomly emitted pulses reach this magnitude, the number of pulses required is about $16000 \times 1652 \approx 2.7 \times 10^{7}$. A far simpler and natural explanation for the peak around $3.4 \mathrm{~ms}$ is substructure in the bursts.

Our simulations suggest that of the observed three log-normal distributions, only the lognormal distribution with peak around $3.4 \mathrm{~ms}$ is clear and significant. The other two, though not an instrumental effect, are nonetheless consistent with emission from a source that emits FRBs 

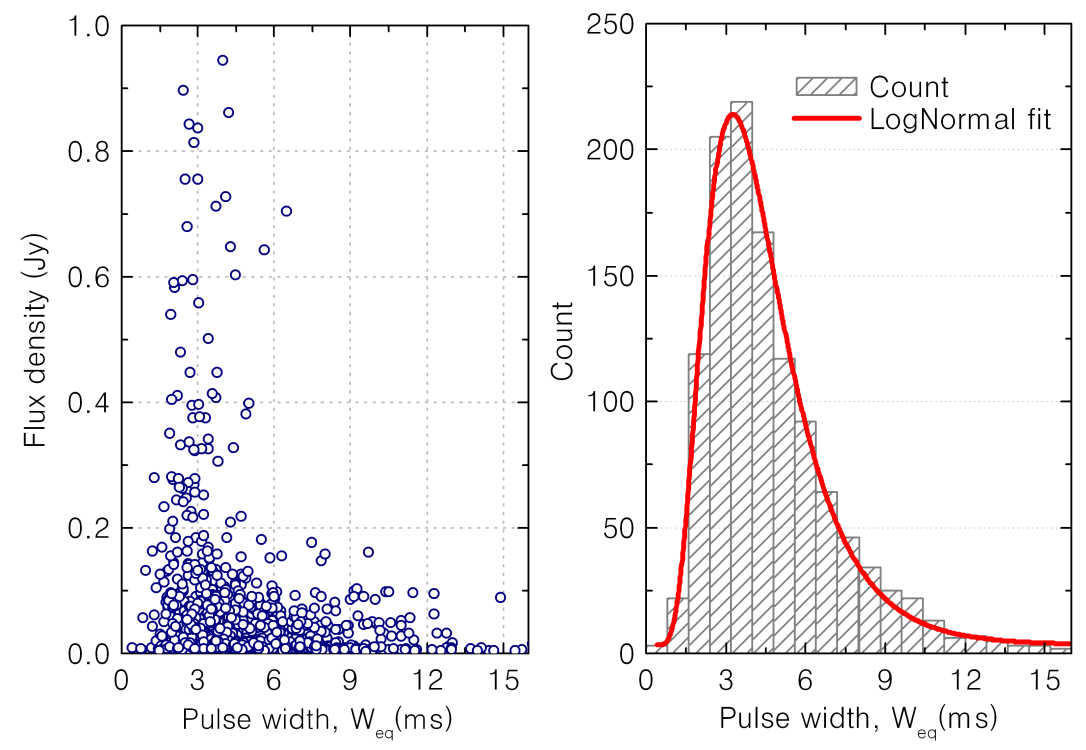

Figure 8: Left: Flux intensity against pulse width for the FRB 121102 bursts in our sample. Right: The equivalent pulse width histogram.

randomly or other "masking" factors, such as the rotating attitudes 14.

Periodicity search The Lomb-Scargle periodogram (LSP) method ${ }^{4041}$ is widely used to identify periodicity in data. It reduces the impact of irregular sampling and highlights any periodic or quasiperiodic features that may exist in the data. We apply the LSP method to the ToAs of FRB121102 to determine if there is a possible period. If the bursts of FRB121102 do have a period, folding the burst arrival times according to this period would gather ToAs into one phase bin, or into a phase range if a quasi-periodic oscillation exists.

Fig. 10 shows the periodogram of FRB121102 bursts from $0.001 \mathrm{~s}$ to $100 \mathrm{~d}$. The left six figures are in timescales from $100 \mathrm{~d}$ to $0.01 \mathrm{~d}$, and the right six figures are in timescale from $1000 \mathrm{~s}$ to $0.001 \mathrm{~s}$. The daily observational effect produces artificial peaks around $1 \mathrm{~d}$ and its higher 

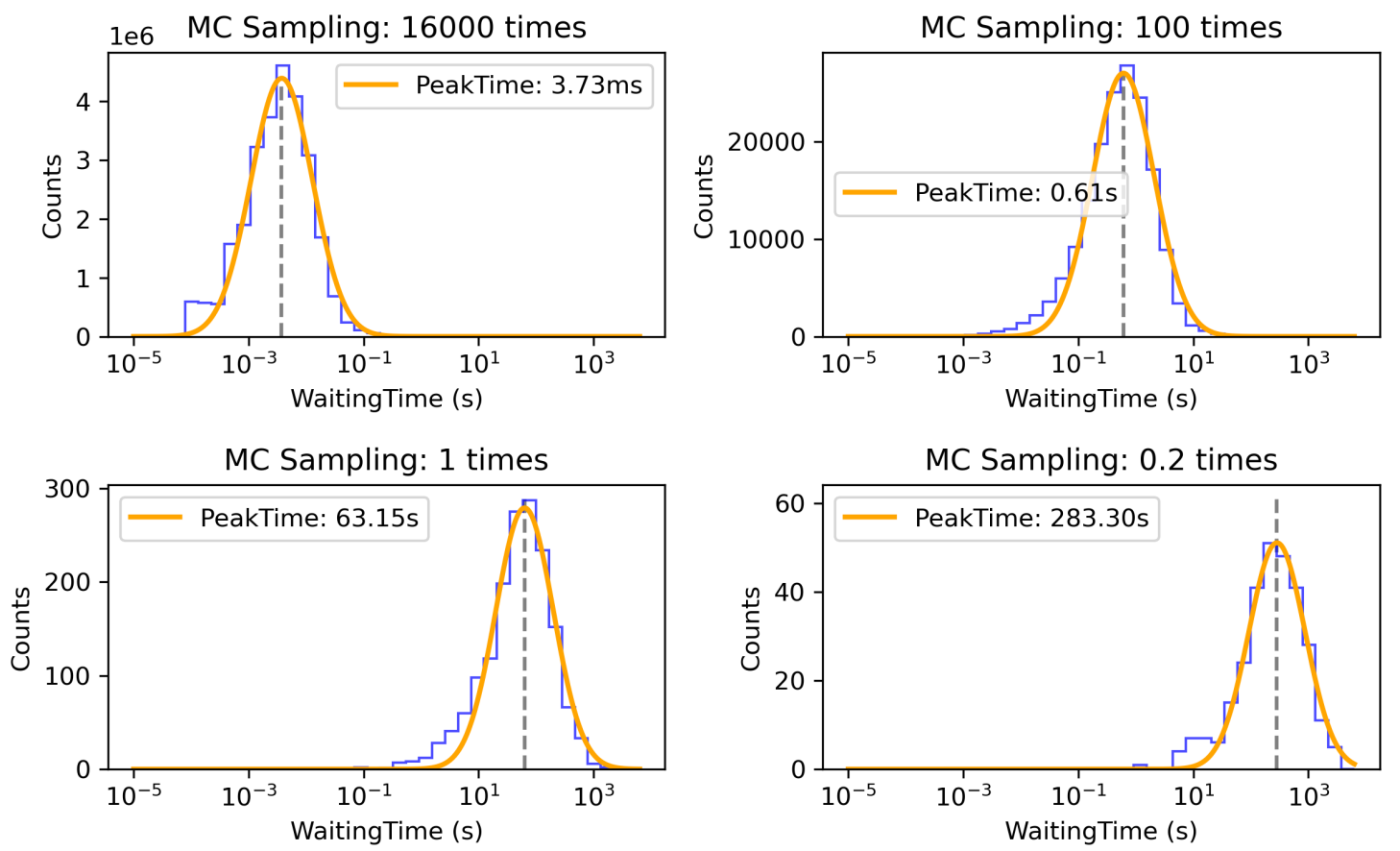

Figure 9: MC simulations of the waiting time distribution. These four figures show the distribution of waiting time sampled according to 16000 times, 100 times, 1 time, and 0.2 times of the real pulse number. The peak times of the four log-normal distributions are $3.7 \mathrm{~ms}, 0.61 \mathrm{~s}, 63.15 \mathrm{~s}$, 283.3 s, respectively.

harmonics in the figure. There are peaks around $63 \mathrm{~s}$ and $736 \mathrm{~s}$, however, folding the burst ToAs at that trial period did not produce a significant bunching in phase (Fig. 10: bottom).

Applying the LSP method to the ToAs of FRB 121102 for period searching, we did not find any effective period within the total observation duration. We also tried traversing the period $(P)$ and period derivative $(\dot{P})$ to fold all the pulses, but did not find an obvious period, either. Additionally, we divided the pulses according to energy, and found that the pulses in different energy intervals do not have significant periodicity. And, all observing session falls in the predicted 
active phase of FRB $121102^{42}$, thus the addition of this sample does not alter the $157 \mathrm{~d}$ period found there.
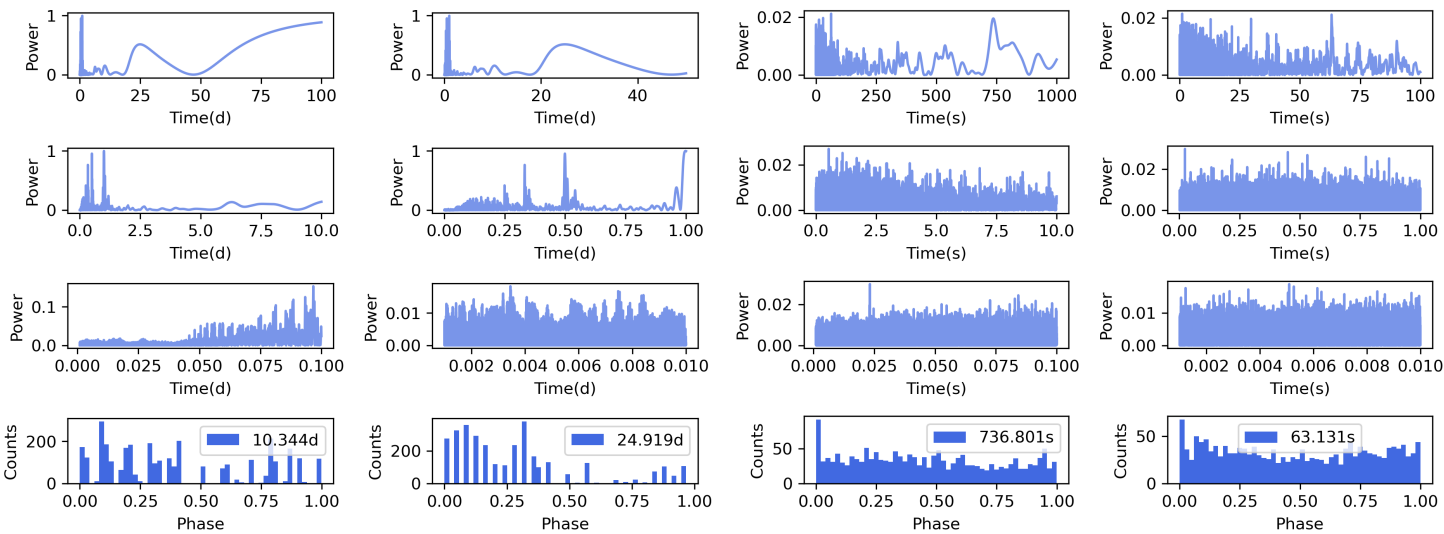

Figure 10: Lomb-Scargle periodogram of FRB121102 burst arrival times. Left: Day periodogram of FRB1211021. Right: Second periodogram of FRB121102.

DM variability The combined effect of sometimes bandwidth-limited structure of FRB bursts and RFI, particularly the satellite bands around $1.2 \mathrm{GHz}$, affects the actual sensitivity. The representative 7- $\sigma$ detection threshold in the FAST campaign is $0.015 \mathrm{Jy}-\mathrm{ms}$ assuming a $1 \mathrm{~ms}$ wide burst in terms of integrated flux (fluence), which is three times lower than that of Arecibo and consistent with a $200 \mathrm{MHz}$ usable bandwidth.

Before we can study the detailed emission characteristics of the bursts, the optimum dispersion measure (DM) should be determined. De-dispersed pulse profiles were created for each DM trial between 500 to $650 \mathrm{pc} \mathrm{cm}^{-3}$ with a step size of $0.05 \mathrm{pc} \mathrm{cm}^{-3}$, using the single pulse search tools in PRESTO 34 . Gaussians (multiple when necessary) were fitted to the profiles. The derivative of each Gaussian was then squared. For multiple components, the squared profiles were summed. 
The optimum DM was then identified according to the maximization of the area under the squared derivative profiles, thus maximizing the structures in the frequency integrated burst profile. The typical DM optimization method and de-dispersed profiles are demonstrated in Fig. 11 .
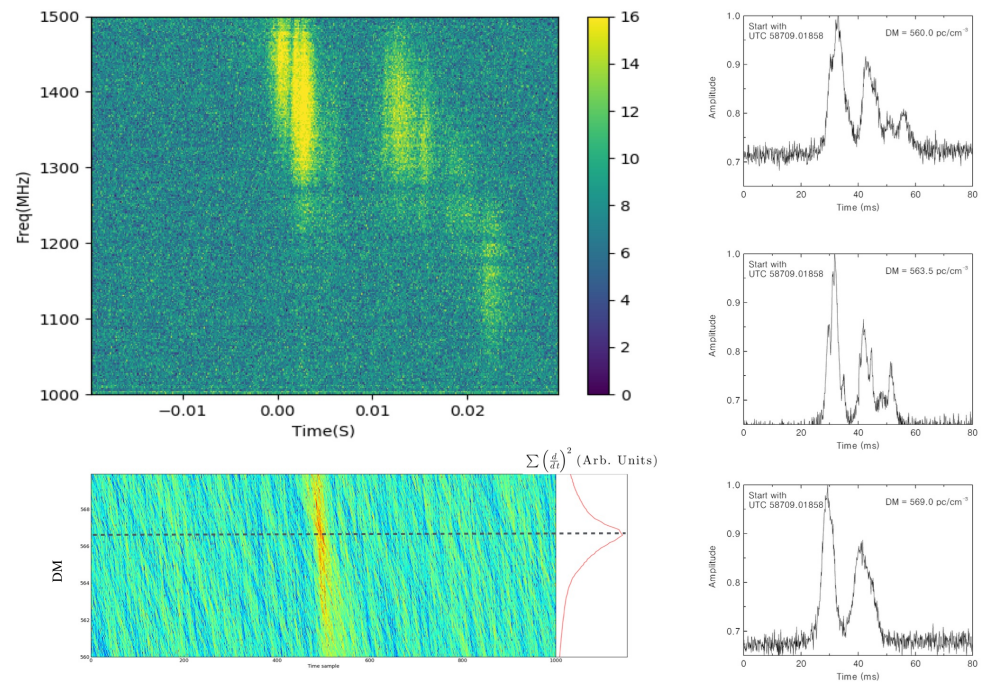

Figure 11: Example of the DM optimization method for FRB 121102. The complex time-frequency structures for the burst of MJD 58729.01858 was revealed with an optimal DM of $563.5 \mathrm{pc} \mathrm{cm}^{-3}$.

The resulting histogram distribution of DMs are shown in Fig. 12, the optimal value is $565.8 \pm 0.3 \pm 0.76 \mathrm{pc} \mathrm{cm}^{-3}$ between MJD 58,724 and MJD 58,776. The two uncertainties are statistical error and system error. The latter is estimated by measuring the $\Delta \mathrm{DM}$ that results in a DM time delay across the whole band equal to half the equivalent width of the bursts (the typical value is $1.5 \mathrm{~ms})$. This suggests that the DM of FRB 121102 has increased by $5-8 \mathrm{pc} \mathrm{cm}^{-3}(1-1.4 \%)$ with more than $20 \sigma$ significance compared with earlier detections from MJD 57,364, where Scholz et al. ${ }^{15}$ found the optimal value to be $558.6 \pm 0.3 \pm 1.4 \mathrm{pc} \mathrm{cm}^{-3}$ with the similar methodology. Fur- 
thermore, the measured DM values and their uncertainties of the bursts are shown in Fig. 4 as a function of individual observations. DM seems to be varying with epochs and has increased over the last 6 years based on the statistics of Ref. 16 and http://www.frbcat.org. The DM value $565.8 \pm 0.3 \pm 0.76 \mathrm{pc} \mathrm{cm}^{-3}$ from our study is consistent with a DM growth rate with a linear fit

$$
\frac{d \mathrm{DM}}{d t}=+0.85 \pm 0.10 \mathrm{pc} \mathrm{cm}^{-3} \mathrm{yr}^{-1}
$$

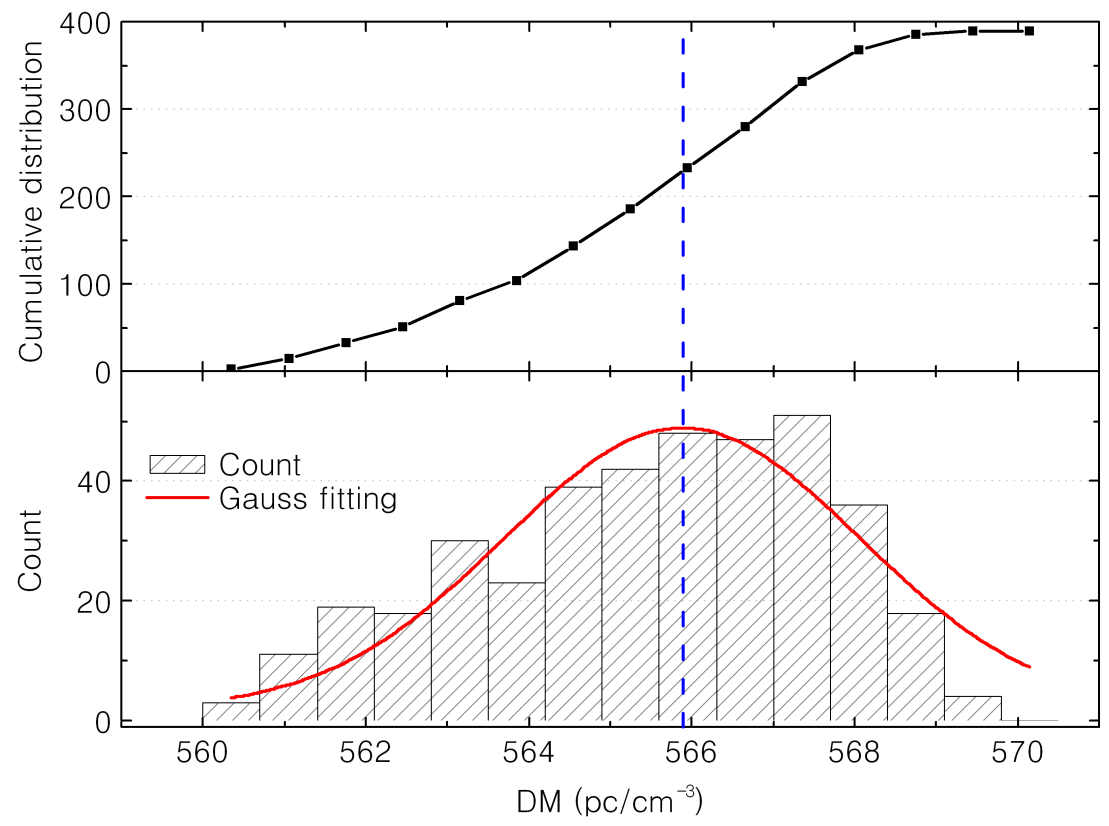

Figure 12: Histogram and cumulative distribution of dispersion measure for FRB 121102. 
33. Petroff, E., Bailes, M., Barr, E. D., Bhat, N. D. R., Bian, F., Burke-Spolaor, S., Caleb, M., Champion, D., Chandra, P., Da Costa, G., Delvaux, C., Flynn, C., Gehrels, N., Greiner, J., Jameson, A., Johnston, S., Kasliwal, M. M., Keane, E. F., Keller, S., Kocz, J., Kramer, M., Leloudas, G., Malesani, D., Mulchaey, J. S., Ng, C., Ofek, E. O., Perley, D. A., Possenti, A., Schmidt, B.P., Shen, Yue, Stappers, B., Tisserand, P., van Straten, W., Wolf, C. A real-time fast radio burst: polarization detection and multiwavelength follow-up. MNRAS 447, 246 (2015).

34. Ransom, S. M. New search techniques for binary pulsars. , Ph.D. Thesis (2001).

35. Zhang, B. Fast Radio Burst Energetics and Detectability from High Redshifts. ApJL 867, L21 (2018).

36. Luo, R., Lee, K., Lorimer, D. R. \& Zhang, B. On the normalized FRB luminosity function. MNRAS 481, 2320-2337 (2018).

37. Lu, W., Piro, Anthony L., Implications from ASKAP Fast Radio Burst Statistics. ApJ 883, 3796-3847 (2019).

38. Luo, R., Men, Y., Lee, K., Wang, W., Lorimer, D. R. \& Zhang, B. On the FRB luminosity function - - II. Event rate density. MNRAS 494, 665-679 (2020).

39. The CHIME/FRB Collaboration et al. CHIME/FRB Detection of Eight New Repeating Fast Radio Burst Sources. arXiv e-prints (2019). 1908.03507.

40. Lomb, N. R. Least-Squares Frequency Analysis of Unequally Spaced Data. Ap\&SS 39, 447-462 (1976). 
41. Scargle, J. D. Studies in astronomical time series analysis. II. Statistical aspects of spectral analysis of unevenly spaced data. ApJ 263, 835-853 (1982).

42. Rajwade, K. M., Mickaliger, M. B., Stappers, B. W., Morello, V., Agarwal, D., Bassa, C. G., Breton, R. P., Caleb, M., Karastergiou, A., Keane, E. F. \& Lorimer, D. R. Possible periodic activity in the repeating FRB 121102. MNRAS 495, 3551-3558 (2020).

43. Cruces, M., Spitler, L. G., Scholz, P., Lynch, R., Seymour, A., Hessels, J. W. T., Gouiffès, C., Hilmarsson, G. H., Kramer, M., Munjal, S., Repeating behaviour of FRB 121102: periodicity, waiting times and energy distribution. (2020). 2008.03461. 
Extended Data Table1: The properties of the 1652 FRB121102 bursts detected by FAST.

Table 1:

\begin{tabular}{|c|c|c|c|c|c|c|c|}
\hline Burst ID & $\begin{array}{c}\text { Burst time }^{a)} \\
\text { (MJD) }\end{array}$ & $\begin{array}{c}\mathrm{DM} \\
\left(\mathrm{pc} \mathrm{cm}^{-3}\right)\end{array}$ & $\begin{array}{l}\begin{array}{l}\text { Width } \\
\text { (ms) }\end{array}\end{array}$ & $\begin{array}{c}\begin{array}{c}\text { Bandwidth }^{b)} \\
(\mathrm{GHz})\end{array} \\
\end{array}$ & $\begin{array}{c}\begin{array}{c}\text { Peak Flux } \\
\text { (mJy) }\end{array}\end{array}$ & $\begin{array}{c}\begin{array}{c}\text { Fluence } \\
\text { (Jy ms) }\end{array} \\
\text { (Jy }\end{array}$ & $\begin{array}{c}\text { Energy } \\
\text { (erg) }\end{array}$ \\
\hline 1 & 58724.877574724 & $567.3(12)$ & $6.13(9)$ & 0.11 & $12.831 \pm 0.2$ & $0.0787(2)$ & $8.426(16) \times 10^{37}$ \\
\hline 2 & 58724.879789116 & $564.3(7)$ & $4.0(3)$ & 0.25 & $28.25 \pm 1.7$ & $0.1130(0)$ & $1.210(0) \times 10^{38}$ \\
\hline 3 & 58724.885144007 & $565.1(3)$ & $2.83(0)$ & 0.4 & $269.05 \pm 1.9$ & $0.7614(0)$ & $8.151(0) \times 10^{38}$ \\
\hline 4 & 58724.886345270 & $568.3(5)$ & $6.35(9)$ & 0.26 & $7.003 \pm 0.1$ & $0.0445(1)$ & $4.764(8) \times 10^{37}$ \\
\hline 5 & 58724.886384602 & $565.8(5)$ & $8.51(9)$ & 0.16 & $6.268 \pm 0.1$ & 0.0534 ( 1$)$ & $5.711(9) \times 10^{37}$ \\
\hline 6 & 58724.887370014 & $568.8(5)$ & $4.25(6)$ & 0.12 & $4.474 \pm 0.1$ & $0.0190(0)$ & $2.036(3) \times 10^{37}$ \\
\hline 7 & 58724.887596868 & $567.6(6)$ & $5.49(7)$ & 0.2 & $12.48 \pm 1.3$ & $0.0685(1)$ & $7.334(8) \times 10^{37}$ \\
\hline 8 & 58724.891114144 & $565.8(5)$ & $7.99(9)$ & 0.23 & $7.943 \pm 0.1$ & $0.0635(1)$ & $6.796(10) \times 10^{37}$ \\
\hline 9 & 58724.902732246 & $567.6(5)$ & $6.52(8)$ & 0.1 & $8.97 \pm 1.0$ & 0.0585 (1) & $6.261(8) \times 10^{37}$ \\
\hline 10 & 58724.905970614 & $564.2(2)$ & $3.5(1)$ & 0.4 & $21.05 \pm 1.2$ & $0.0737(0)$ & $7.887(0) \times 10^{37}$ \\
\hline 11 & 58724.905970706 & 564.7 ( 3 ) & $5.35(4)$ & 0.4 & $102.48 \pm 1.5$ & 0.5483 ( 1 ) & $5.869(0) \times 10^{38}$ \\
\hline 12 & 58724.907803711 & $565.7(7)$ & $4.49(5)$ & 0.22 & $15.7 \pm 1.4$ & $0.0705(1)$ & $7.546(6) \times 10^{37}$ \\
\hline 13 & 58724.909622982 & 565.7 ( 6 ) & $8.54(5)$ & 0.15 & $17.99 \pm 0.9$ & $0.1536(0)$ & $1.645(0) \times 10^{38}$ \\
\hline 14 & 58724.911838133 & $565.3(6)$ & $9.03(8)$ & 0.2 & $12.86 \pm 0.9$ & 0.1161 (1) & $1.243(0) \times 10^{38}$ \\
\hline 15 & 58724.915007440 & $565.1(6)$ & $9.61(8)$ & 0.2 & $13.47 \pm 0.9$ & $0.1295(1)$ & $1.386(0) \times 10^{38}$ \\
\hline 16 & 58724.916569615 & $564.2(3)$ & $2.91(2)$ & 0.3 & $23.49 \pm 1.6$ & $0.0684(0)$ & $7.317(3) \times 10^{37}$ \\
\hline 17 & 58724.917195581 & $567.3(5)$ & $5.38(3)$ & 0.22 & $22.7 \pm 1.2$ & $0.1221(0)$ & $1.307(0) \times 10^{38}$ \\
\hline 18 & 58724.917196236 & $565.0(6)$ & $8.08(4)$ & 0.22 & $25.1 \pm 1.0$ & $0.2028(0)$ & $2.171(0) \times 10^{38}$ \\
\hline 19 & 58724.920837633 & $566.1(7)$ & $1.51(4)$ & 0.3 & $10.64 \pm 2.4$ & 0.0161 (1) & $1.720(11) \times 10^{37}$ \\
\hline 20 & 58724.922249805 & $565.4(4)$ & $2.32(1)$ & 0.29 & $35.14 \pm 1.7$ & $0.0815(0)$ & $8.727(2) \times 10^{37}$ \\
\hline 21 & 58724.922304628 & $567.1(6)$ & $2.43(3)$ & 0.15 & $20.64 \pm 1.9$ & $0.0502(1)$ & $5.369(5) \times 10^{37}$ \\
\hline 22 & 58724.922304888 & $565.2(3)$ & $1.89(0)$ & 0.1 & $31.7 \pm 1.7$ & $0.0599(0)$ & $6.414(0) \times 10^{37}$ \\
\hline 23 & 58724.922304919 & $566.0(3)$ & $6.37(4)$ & 0.4 & $198.2 \pm 3.2$ & $1.2625(1)$ & $1.352(0) \times 10^{39}$ \\
\hline 24 & 58724.923026148 & $567.4(4)$ & $9.12(8)$ & 0.21 & $12.11 \pm 0.9$ & $0.1104(1)$ & $1.182(0) \times 10^{38}$ \\
\hline 25 & 58724.924419439 & $563.9(4)$ & $4.68(6)$ & 0.295 & $11.22 \pm 1.2$ & $0.0525(1)$ & $5.621(7) \times 10^{37}$ \\
\hline 26 & 58724.924419988 & $564.7(2)$ & $3.28(3)$ & 0.3 & $17.02 \pm 1.4$ & $0.0558(0)$ & $5.976(4) \times 10^{37}$ \\
\hline 27 & 58724.925545347 & $566.0(5)$ & $3.08(4)$ & 0.15 & $12.327 \pm 0.1$ & 0.0379 (1) & $4.058(5) \times 10^{37}$ \\
\hline 28 & 58724.929142015 & $564.3(5)$ & $3.35(5)$ & 0.19 & $5.409 \pm 0.1$ & $0.0181(0)$ & $1.940(2) \times 10^{37}$ \\
\hline 29 & 58724.930911228 & $564.5(5)$ & $5.75(4)$ & 0.21 & $15.98 \pm 0.9$ & $0.0919(0)$ & $9.836(3) \times 10^{37}$ \\
\hline 30 & 58724.931130179 & $566.9(7)$ & $8.1(6)$ & 0.11 & $12.76 \pm 0.8$ & $0.1034(0)$ & $1.106(0) \times 10^{38}$ \\
\hline 31 & 58724.933070175 & $567.6(3)$ & $4.74(4)$ & 0.19 & $15.08 \pm 1.0$ & $0.0715(0)$ & $7.652(4) \times 10^{37}$ \\
\hline 32 & 58724.933262744 & $564.1(3)$ & $2.01(1)$ & 0.32 & $58.5 \pm 1.4$ & $0.1176(0)$ & $1.259(0) \times 10^{38}$ \\
\hline 33 & 58724.933455784 & $566.5(6)$ & $12.9(8)$ & 0.26 & $12.95 \pm 0.6$ & $0.1671(0)$ & $1.788(0) \times 10^{38}$ \\
\hline 34 & 58724.934141764 & $568.8(5)$ & $8.81(9)$ & 0.07 & $2.872 \pm 0.0$ & $0.0253(0)$ & $2.709(3) \times 10^{37}$ \\
\hline 35 & 58724.935844282 & $565.9(5)$ & $5.96(6)$ & 0.13 & $8.38 \pm 0.7$ & $0.0499(0)$ & $5.347(4) \times 10^{37}$ \\
\hline 36 & 58724.937217213 & $564.8(5)$ & $6.79(9)$ & 0.1 & $15.709 \pm 0.2$ & $0.1067(1)$ & $1.142(1) \times 10^{38}$ \\
\hline 37 & 58724.937990740 & $565.5(4)$ & $3.75(1)$ & 0.19 & $35.39 \pm 1.1$ & $0.1327(0)$ & $1.421(0) \times 10^{38}$ \\
\hline 38 & 58724.939026312 & $565.3(6)$ & $1.92(2)$ & 0.19 & $20.0 \pm 1.6$ & $0.0384(0)$ & $4.111(3) \times 10^{37}$ \\
\hline 39 & 58724.939193506 & $565.8(5)$ & $7.78(7)$ & 0.14 & $10.75 \pm 0.8$ & $0.0836(1)$ & $8.953(5) \times 10^{37}$ \\
\hline 40 & 58724.942386480 & $567.8(5)$ & $7.23(9)$ & 0.09 & $14.683 \pm 0.1$ & $0.1062(1)$ & $1.137(1) \times 10^{38}$ \\
\hline 41 & 58724.942504340 & $568.1(1)$ & $5.41(5)$ & 0.23 & $13.15 \pm 1.0$ & $0.0711(0)$ & $7.616(5) \times 10^{37}$ \\
\hline 42 & 58724.943999917 & $568.6(7)$ & $7.46(4)$ & 0.31 & $21.23 \pm 0.9$ & $0.1584(0)$ & $1.695(0) \times 10^{38}$ \\
\hline 43 & 58724.944237051 & $566.9(4)$ & $6.39(7)$ & 0.31 & $10.28 \pm 0.9$ & 0.0657 ( 1 ) & $7.032(6) \times 10^{37}$ \\
\hline 44 & 58724.945983368 & $560.8(5)$ & $4.12(6)$ & 0.12 & $14.0 \pm 0.1$ & 0.0577 ( 1 ) & $6.179(7) \times 10^{37}$ \\
\hline 45 & 58724.947858821 & $557.3(5)$ & $4.61(7)$ & 0.25 & $7.726 \pm 0.1$ & $0.0356(0)$ & $3.811(4) \times 10^{37}$ \\
\hline 46 & 58724.949430947 & $565.7(4)$ & $3.1(2)$ & 0.19 & $22.26 \pm 1.3$ & $0.0690(0)$ & $7.387(3) \times 10^{37}$ \\
\hline 47 & 58724.952853352 & $564.1(6)$ & $1.5(2)$ & 0.19 & $24.48 \pm 2.0$ & $0.0367(0)$ & $3.931(3) \times 10^{37}$ \\
\hline 48 & 58724.952853391 & $564.1(5)$ & $3.8(3)$ & 0.17 & $27.18 \pm 1.2$ & $0.1033(0)$ & $1.106(0) \times 10^{38}$ \\
\hline 49 & 58724.952934161 & $567.1(6)$ & $5.86(5)$ & 0.32 & $14.47 \pm 0.9$ & $0.0848(0)$ & $9.077(4) \times 10^{37}$ \\
\hline 50 & 58724.953352885 & $564.0(4)$ & $2.49(2)$ & 0.3 & $22.33 \pm 1.4$ & $0.0556(0)$ & $5.952(2) \times 10^{37}$ \\
\hline 51 & 58724.953924721 & $565.8(4)$ & $2.27(3)$ & 0.31 & $15.93 \pm 1.5$ & $0.0362(0)$ & $3.871(3) \times 10^{37}$ \\
\hline 52 & 58724.955146206 & $563.4(5)$ & $2.49(1)$ & 0.2 & $50.33 \pm 1.4$ & $0.1253(0)$ & $1.342(0) \times 10^{38}$ \\
\hline 53 & 58724.956157021 & $568.0(6)$ & $4.6(4)$ & 0.19 & $14.13 \pm 1.0$ & $0.0650(0)$ & $6.958(4) \times 10^{37}$ \\
\hline 54 & 58724.956611826 & $565.4(5)$ & $2.13(2)$ & 0.16 & $25.57 \pm 1.5$ & $0.0545(0)$ & $5.830(2) \times 10^{37}$ \\
\hline 55 & 58724.960656516 & $567.5(9)$ & $3.44(6)$ & 0.16 & $9.46 \pm 1.2$ & 0.0325 ( 1 ) & $3.484(7) \times 10^{37}$ \\
\hline 56 & 58724.960656588 & $567.6(9)$ & $4.26(6)$ & 0.16 & $26.9 \pm 1.9$ & $0.1146(1)$ & $1.227(1) \times 10^{38}$ \\
\hline 57 & 58724.960656635 & $568.2(10)$ & $3.75(4)$ & 0.18 & $28.36 \pm 3.0$ & $0.1064(1)$ & $1.138(1) \times 10^{38}$ \\
\hline
\end{tabular}


Table 1:

\begin{tabular}{|c|c|c|c|c|c|c|c|}
\hline Burst ID & $\begin{array}{c}\text { Burst time }^{a)} \\
\text { (MJD) }\end{array}$ & $\begin{array}{c}\mathrm{DM} \\
\left(\mathrm{pc} \mathrm{cm}^{-3}\right)\end{array}$ & $\begin{array}{l}\text { Width } \\
\text { (ms) }\end{array}$ & $\begin{array}{c}\text { Bandwidth }^{b)} \\
(\mathrm{GHz})\end{array}$ & $\begin{array}{c}\text { Peak Flux } \\
(\mathrm{mJy})\end{array}$ & $\begin{array}{c}\begin{array}{c}\text { Fluence } \\
(\mathrm{Jy} \mathrm{ms})\end{array} \\
\end{array}$ & $\begin{array}{c}\text { Energy } \\
\text { (erg) }\end{array}$ \\
\hline 58 & 58724.962651274 & $565.8(5)$ & $5.63(8)$ & 0.13 & $16.295 \pm 0.1$ & $0.0918(1)$ & $9.825(9) \times 10^{37}$ \\
\hline 59 & 58724.969017661 & $566.0(5)$ & $3.21(5)$ & 0.11 & $9.323 \pm 0.1$ & $0.0299(0)$ & $3.205(3) \times 10^{37}$ \\
\hline 60 & 58724.970650465 & $566.8(6)$ & $9.83(9)$ & 0.17 & $7.51 \pm 0.7$ & $0.0738(1)$ & $7.903(7) \times 10^{37}$ \\
\hline 61 & 58724.970715464 & $568.6(6)$ & $6.67(5)$ & 0.17 & $11.87 \pm 0.8$ & $0.0792(0)$ & $8.475(4) \times 10^{37}$ \\
\hline 62 & 58724.971621807 & $567.8(6)$ & $6.49(6)$ & 0.22 & $10.61 \pm 0.8$ & $0.0689(0)$ & $7.371(4) \times 10^{37}$ \\
\hline 63 & 58724.971795673 & $566.3(4)$ & $3.02(2)$ & 0.2 & $23.82 \pm 1.1$ & $0.0719(0)$ & $7.701(2) \times 10^{37}$ \\
\hline 64 & 58724.973164234 & $565.8(5)$ & $2.97(1)$ & 0.4 & $38.96 \pm 1.2$ & $0.1157(0)$ & $1.239(0) \times 10^{38}$ \\
\hline 65 & 58724.973294692 & $566.0(5)$ & $3.45(5)$ & 0.15 & $8.339 \pm 0.1$ & $0.0288(0)$ & $3.084(2) \times 10^{37}$ \\
\hline 66 & 58724.973315882 & $565.0(3)$ & $3.1(4)$ & 0.12 & $13.98 \pm 2.0$ & $0.0433(1)$ & $4.639(9) \times 10^{37}$ \\
\hline 67 & 58724.973430444 & $565.9(6)$ & $6.01(6)$ & 0.19 & $9.94 \pm 0.8$ & $0.0597(0)$ & $6.395(4) \times 10^{37}$ \\
\hline 68 & 58724.974034678 & $568.0(7)$ & $2.71(6)$ & 0.19 & $6.92 \pm 1.3$ & 0.0187 ( 1 ) & $2.008(8) \times 10^{37}$ \\
\hline 69 & 58724.974622802 & $568.0(7)$ & $4.86(3)$ & 0.28 & $16.43 \pm 0.9$ & $0.0799(0)$ & $8.548(3) \times 10^{37}$ \\
\hline 70 & 58724.975333910 & $564.9(5)$ & $3.42(2)$ & 0.16 & $17.49 \pm 1.0$ & $0.0598(0)$ & $6.403(2) \times 10^{37}$ \\
\hline 71 & 58724.975827187 & $567.2(8)$ & $4.0(2)$ & 0.21 & $22.28 \pm 0.9$ & $0.0891(0)$ & $9.540(1) \times 10^{37}$ \\
\hline 72 & 58724.976403537 & $564.0(12)$ & $5.19(3)$ & 0.19 & $16.14 \pm 0.8$ & $0.0838(0)$ & $8.967(2) \times 10^{37}$ \\
\hline 73 & 58724.979800125 & $566.8(4)$ & $3.77(2)$ & 0.39 & $17.91 \pm 1.0$ & $0.0675(0)$ & $7.228(2) \times 10^{37}$ \\
\hline 74 & 58724.981515494 & $565.6(3)$ & $2.55(2)$ & 0.13 & $18.75 \pm 1.0$ & $0.0478(0)$ & $5.118(1) \times 10^{37}$ \\
\hline 75 & 58724.981515628 & $565.2(2)$ & $4.38(5)$ & 0.21 & $8.07 \pm 0.8$ & $0.0353(0)$ & $3.784(4) \times 10^{37}$ \\
\hline 76 & 58724.983679670 & $566.0(5)$ & $3.31(5)$ & 0.15 & $8.13 \pm 0.1$ & $0.0269(0)$ & $2.882(2) \times 10^{37}$ \\
\hline 77 & 58724.987275493 & $564.8(5)$ & $8.53(9)$ & 0.205 & $4.211 \pm 0.0$ & $0.0359(0)$ & $3.844(3) \times 10^{37}$ \\
\hline 78 & 58724.987452304 & $566.0(5)$ & $2.58(4)$ & 0.07 & $6.591 \pm 0.1$ & $0.0170(0)$ & $1.823(1) \times 10^{37}$ \\
\hline 79 & 58724.987467774 & $564.5(4)$ & $3.2(4)$ & 0.4 & $10.29 \pm 1.0$ & $0.0329(0)$ & $3.525(4) \times 10^{37}$ \\
\hline 80 & 58724.987468103 & $563.8(2)$ & $5.52(6)$ & 0.4 & $7.69 \pm 0.8$ & $0.0425(1)$ & $4.544(5) \times 10^{37}$ \\
\hline 81 & 58724.989651176 & $563.8(2)$ & $4.26(2)$ & 0.205 & $28.96 \pm 1.0$ & $0.1234(0)$ & $1.321(0) \times 10^{38}$ \\
\hline 82 & 58724.990468879 & $564.6(4)$ & $2.54(1)$ & 0.31 & $29.24 \pm 1.2$ & $0.0743(0)$ & $7.950(1) \times 10^{37}$ \\
\hline 83 & 58724.992182123 & $563.8(4)$ & $5.13(5)$ & 0.36 & $9.33 \pm 0.7$ & $0.0479(0)$ & $5.124(3) \times 10^{37}$ \\
\hline 84 & 58724.993445518 & $567.1(3)$ & $4.92(3)$ & 0.36 & $11.61 \pm 0.7$ & $0.0571(0)$ & $6.115(2) \times 10^{37}$ \\
\hline 85 & 58724.995190620 & $568.6(4)$ & $1.82(2)$ & 0.36 & $24.35 \pm 2.4$ & $0.0443(0)$ & $4.744(4) \times 10^{37}$ \\
\hline 86 & 58724.995190653 & $568.0(3)$ & $4.88(4)$ & 0.16 & $24.01 \pm 0.8$ & $0.1172(0)$ & $1.254(0) \times 10^{38}$ \\
\hline 87 & 58724.997333743 & $567.8(5)$ & $3.42(5)$ & 0.18 & $14.924 \pm 0.1$ & $0.0510(0)$ & $5.463(4) \times 10^{37}$ \\
\hline 88 & 58725.876003233 & $567.1(6)$ & $2.85(3)$ & 0.25 & $130.23 \pm 9.7$ & $0.3712(2)$ & $3.973(2) \times 10^{38}$ \\
\hline 89 & 58725.876225404 & $562.3(5)$ & $6.13(9)$ & 0.21 & $4.462 \pm 0.1$ & $0.0274(1)$ & $2.928(5) \times 10^{37}$ \\
\hline 90 & 58725.880203168 & $563.0(7)$ & 1.77 ( 3 ) & 0.35 & $84.73 \pm 11.7$ & $0.1500(4)$ & $1.605(4) \times 10^{38}$ \\
\hline 91 & 58725.880203207 & $566.9(5)$ & $2.93(4)$ & 0.3 & $90.8 \pm 9.4$ & $0.2660(4)$ & $2.848(4) \times 10^{38}$ \\
\hline 92 & 58725.881791164 & $565.9(5)$ & $6.26(9)$ & 0.29 & $6.499 \pm 0.1$ & $0.0407(1)$ & $4.355(7) \times 10^{37}$ \\
\hline 93 & 58725.885653541 & $566.0(5)$ & $3.84(6)$ & 0.15 & $14.537 \pm 0.2$ & $0.0559(1)$ & $5.981(9) \times 10^{37}$ \\
\hline 94 & 58725.885758410 & $565.4(5)$ & 3.18 ( 2 ) & 0.2 & $144.65 \pm 8.2$ & $0.4600(2)$ & $4.924(1) \times 10^{38}$ \\
\hline 95 & 58725.887654087 & $566.0(5)$ & $3.47(5)$ & 0.29 & $33.86 \pm 0.5$ & $0.1175(2)$ & $1.258(2) \times 10^{38}$ \\
\hline 96 & 58725.887654101 & $565.7(5)$ & $3.44(2)$ & 0.2 & $132.81 \pm 7.6$ & $0.4569(2)$ & $4.891(1) \times 10^{38}$ \\
\hline 97 & 58725.888099642 & $566.0(5)$ & $3.01(4)$ & 0.23 & $33.251 \pm 0.5$ & $0.1001(2)$ & $1.072(1) \times 10^{38}$ \\
\hline 98 & 58725.888099645 & $566.1(7)$ & $2.83(2)$ & 0.1 & $122.03 \pm 8.8$ & $0.3453(2)$ & $3.697(2) \times 10^{38}$ \\
\hline 99 & 58725.888616940 & $565.0(5)$ & $7.37(9)$ & 0.26 & $21.735 \pm 0.3$ & $0.1601(3)$ & $1.714(2) \times 10^{38}$ \\
\hline 100 & 58725.888616945 & $564.1(2)$ & 3.98 ( 3 ) & 0.1 & $105.27 \pm 7.5$ & $0.4190(2)$ & $4.485(2) \times 10^{38}$ \\
\hline 101 & 58725.889445554 & $564.5(3)$ & $2.03(2)$ & 0.1 & $144.07 \pm 10.3$ & $0.2925(2)$ & $3.131(1) \times 10^{38}$ \\
\hline 102 & 58725.890731808 & $566.1(4)$ & $4.44(6)$ & 0.1 & $62.65 \pm 6.8$ & $0.2782(4)$ & $2.978(4) \times 10^{38}$ \\
\hline 103 & 58725.893190360 & $564.5(4)$ & $3.12(3)$ & 0.1 & $97.59 \pm 7.8$ & $0.3045(2)$ & $3.259(2) \times 10^{38}$ \\
\hline 104 & 58725.896969716 & $559.7(5)$ & $3.66(5)$ & 0.18 & $20.889 \pm 0.2$ & $0.0766(1)$ & $8.195(11) \times 10^{37}$ \\
\hline 105 & 58725.898777609 & $568.5(5)$ & $8.88(9)$ & 0.13 & $11.459 \pm 0.1$ & 0.1017 ( 1 ) & $1.089(1) \times 10^{38}$ \\
\hline 106 & 58725.900736313 & $566.7(5)$ & $4.92(7)$ & 0.13 & $13.059 \pm 0.1$ & $0.0642(1)$ & $6.877(8) \times 10^{37}$ \\
\hline 107 & 58725.901011470 & $564.7(3)$ & $2.93(2)$ & 0.4 & $184.07 \pm 7.8$ & $0.5393(1)$ & $5.773(1) \times 10^{38}$ \\
\hline 108 & 58725.901011486 & $566.0(5)$ & $3.34(5)$ & 0.45 & $44.469 \pm 0.5$ & $0.1485(2)$ & $1.590(2) \times 10^{38}$ \\
\hline 109 & 58725.901675614 & $566.0(5)$ & 3.37 ( 5 ) & 0.12 & $18.75 \pm 0.2$ & $0.0632(1)$ & $6.768(8) \times 10^{37}$ \\
\hline 110 & 58725.901684753 & $565.4(4)$ & $1.61(1)$ & 0.15 & $169.74 \pm 10.5$ & $0.2733(1)$ & $2.925(1) \times 10^{38}$ \\
\hline 111 & 58725.901684764 & $566.0(5)$ & $1.9(3)$ & 0.13 & $38.466 \pm 0.4$ & $0.0730(1)$ & $7.818(9) \times 10^{37}$ \\
\hline 112 & 58725.902434479 & $566.0(5)$ & $3.48(5)$ & 0.19 & $27.981 \pm 0.3$ & $0.0974(1)$ & $1.043(1) \times 10^{38}$ \\
\hline 113 & 58725.902434492 & $566.5(4)$ & $4.25(4)$ & 0.2 & $90.21 \pm 6.2$ & $0.3834(2)$ & $4.104(2) \times 10^{38}$ \\
\hline 114 & 58725.902874998 & $561.0(5)$ & $3.6(5)$ & 0.18 & $10.232 \pm 0.1$ & $0.0369(0)$ & $3.947(4) \times 10^{37}$ \\
\hline 115 & 58725.903052755 & $562.9(7)$ & $1.2(1)$ & 0.1 & $162.74 \pm 12.0$ & $0.1953(1)$ & $2.091(1) \times 10^{38}$ \\
\hline 116 & 58725.903419641 & $567.2(5)$ & $11.65(9)$ & 0.105 & $8.136 \pm 0.1$ & $0.0948(1)$ & $1.015(1) \times 10^{38}$ \\
\hline 117 & 58725.915557003 & $568.5(5)$ & $5.89(9)$ & 0.2 & $18.22 \pm 0.2$ & $0.1073(2)$ & $1.148(1) \times 10^{38}$ \\
\hline 118 & 58725.916229819 & $569.8(5)$ & $4.26(6)$ & 0.07 & $7.496 \pm 0.1$ & $0.0319(0)$ & $3.420(4) \times 10^{37}$ \\
\hline 119 & 58725.916365197 & $566.0(5)$ & $3.38(5)$ & 0.12 & $9.399 \pm 0.1$ & $0.0318(0)$ & $3.402(4) \times 10^{37}$ \\
\hline 120 & 58725.917651625 & $566.0(5)$ & $3.37(5)$ & 0.105 & $14.154 \pm 0.2$ & $0.0478(1)$ & $5.112(7) \times 10^{37}$ \\
\hline
\end{tabular}


Table 1:

\begin{tabular}{|c|c|c|c|c|c|c|c|}
\hline Burst ID & $\begin{array}{c}\text { Burst time }^{a)} \\
\text { (MJD) }\end{array}$ & $\begin{array}{c}\mathrm{DM} \\
(\mathrm{pc} \mathrm{cm}-3)\end{array}$ & $\begin{array}{l}\text { Width } \\
\text { (ms) }\end{array}$ & $\begin{array}{c}\left.\text { Bandwidth }^{b}\right) \\
(\mathrm{GHz})\end{array}$ & $\begin{array}{c}\begin{array}{c}\text { Peak Flux } \\
(\mathrm{mJy})\end{array} \\
\end{array}$ & $\begin{array}{c}\text { Fluence } \\
\text { (Jy ms) }\end{array}$ & $\begin{array}{c}\text { Energy } \\
\text { (erg) }\end{array}$ \\
\hline 121 & 58725.918913617 & $566.0(5)$ & $3.54(5)$ & 0.2 & $10.218 \pm 0.1$ & $0.0362(1)$ & $3.870(5) \times 10^{37}$ \\
\hline 122 & 58725.920270328 & $566.3(4)$ & $1.92(1)$ & 0.15 & $154.73 \pm 8.4$ & $0.2971(1)$ & $3.180(1) \times 10^{38}$ \\
\hline 123 & 58725.920543411 & $567.7(2)$ & $4.11(5)$ & 0.4 & $60.17 \pm 5.8$ & $0.2473(3)$ & $2.647(2) \times 10^{38}$ \\
\hline 124 & 58725.920543516 & $562.1(4)$ & $3.7(2)$ & 0.4 & $134.23 \pm 6.1$ & $0.4966(1)$ & $5.317(1) \times 10^{38}$ \\
\hline 125 & 58725.921183791 & $566.2(3)$ & $2.42(2)$ & 0.15 & $100.32 \pm 7.4$ & $0.2428(2)$ & $2.599(1) \times 10^{38}$ \\
\hline 126 & 58725.925513631 & $562.8(5)$ & $7.54(9)$ & 0.07 & $9.652 \pm 0.1$ & $0.0728(1)$ & $7.793(10) \times 10^{37}$ \\
\hline 128 & 58725.928638589 & $568.1(5)$ & $5.71(8)$ & 0.15 & $3.3 \pm 0.0$ & $0.0188(0)$ & $2.018(2) \times 10^{37}$ \\
\hline 129 & 58725.928938722 & $564.8(4)$ & $6.12(5)$ & 0.4 & $73.38 \pm 4.8$ & $0.4491(3)$ & $4.807(2) \times 10^{38}$ \\
\hline 130 & 58725.928938767 & $563.7(5)$ & $13.42(9)$ & 0.3 & $11.882 \pm 0.1$ & $0.1594(2)$ & $1.707(2) \times 10^{38}$ \\
\hline 131 & 58725.928938805 & $565.9(5)$ & $3.4(6)$ & 0.4 & $47.6 \pm 6.2$ & $0.1618(4)$ & $1.732(3) \times 10^{38}$ \\
\hline 132 & 58725.929864657 & $563.2(5)$ & $4.54(7)$ & 0.22 & $9.191 \pm 0.1$ & 0.0417 ( 1$)$ & $4.468(5) \times 10^{37}$ \\
\hline 133 & 58725.931323731 & $567.6(5)$ & $7.91(9)$ & 0.29 & $15.082 \pm 0.2$ & $0.1192(2)$ & $1.276(1) \times 10^{38}$ \\
\hline 134 & 58725.932386703 & $566.0(5)$ & $3.81(6)$ & 0.3 & $13.725 \pm 0.1$ & $0.0523(1)$ & $5.593(7) \times 10^{37}$ \\
\hline 135 & 58725.932956010 & $569.4(5)$ & $2.72(4)$ & 0.14 & $8.502 \pm 0.1$ & $0.0231(0)$ & $2.475(3) \times 10^{37}$ \\
\hline 137 & 58725.937982134 & $563.4(8)$ & $2.58(1)$ & 0.1 & $163.74 \pm 7.0$ & $0.4224(1)$ & $4.522(0) \times 10^{38}$ \\
\hline 138 & 58725.937984158 & $564.9(6)$ & $28.3(5)$ & 0.2 & $233.25 \pm 3.1$ & $6.6010(2)$ & $7.066(0) \times 10^{39}$ \\
\hline 139 & 58725.939213347 & $567.7(7)$ & $8.39(4)$ & 0.36 & $81.22 \pm 3.6$ & $0.6814(2)$ & $7.295(1) \times 10^{38}$ \\
\hline 140 & 58725.940506707 & $569.4(5)$ & $3.52(5)$ & 0.28 & $14.28 \pm 0.1$ & $0.0503(1)$ & $5.384(6) \times 10^{37}$ \\
\hline 141 & 58725.940908390 & $566.0(5)$ & $4.04(6)$ & 0.18 & $12.498 \pm 0.1$ & $0.0505(1)$ & $5.404(6) \times 10^{37}$ \\
\hline 142 & 58725.941327953 & $566.0(5)$ & $3.92(6)$ & 0.2 & $17.39 \pm 0.2$ & $0.0682(1)$ & $7.298(8) \times 10^{37}$ \\
\hline 143 & 58725.942245365 & $566.0(5)$ & $2.9(4)$ & 0.21 & $23.407 \pm 0.2$ & $0.0680(1)$ & $7.274(8) \times 10^{37}$ \\
\hline 144 & 58725.943248002 & $563.7(5)$ & $2.41(4)$ & 0.19 & $9.808 \pm 0.1$ & $0.0237(0)$ & $2.535(2) \times 10^{37}$ \\
\hline 145 & 58725.945390254 & $565.4(5)$ & $2.2(3)$ & 0.26 & $34.554 \pm 0.3$ & $0.0759(1)$ & $8.129(9) \times 10^{37}$ \\
\hline 146 & 58725.945538912 & $564.2(2)$ & $2.35(2)$ & 0.15 & $94.99 \pm 7.2$ & $0.2232(1)$ & $2.390(1) \times 10^{38}$ \\
\hline 147 & 58725.945934684 & $566.0(5)$ & $2.95(4)$ & 0.23 & $21.715 \pm 0.2$ & $0.0640(1)$ & $6.855(7) \times 10^{37}$ \\
\hline 148 & 58725.946281650 & $567.6(5)$ & $9.5(9)$ & 0.31 & $10.97 \pm 0.1$ & $0.1042(1)$ & $1.116(1) \times 10^{38}$ \\
\hline 149 & 58725.946967027 & $566.0(5)$ & $3.37(2)$ & 0.2 & $96.28 \pm 5.9$ & $0.3245(1)$ & $3.473(1) \times 10^{38}$ \\
\hline 150 & 58725.946976059 & $562.1(11)$ & $3.94(8)$ & 0.4 & $34.23 \pm 5.3$ & $0.1349(4)$ & $1.444(4) \times 10^{38}$ \\
\hline 151 & 58725.946976137 & $567.7(6)$ & $4.6(9)$ & 0.4 & $33.35 \pm 5.1$ & $0.1534(5)$ & $1.642(4) \times 10^{38}$ \\
\hline 152 & 58725.947440402 & $566.2(6)$ & $3.27(7)$ & 0.25 & $39.02 \pm 5.8$ & $0.1276(4)$ & $1.366(4) \times 10^{38}$ \\
\hline 155 & 58725.951143410 & $564.5(6)$ & $3.88(2)$ & 0.25 & $107.26 \pm 5.5$ & $0.4162(1)$ & $4.455(1) \times 10^{38}$ \\
\hline 156 & 58725.953997841 & $565.7(4)$ & $5.45(4)$ & 0.2 & $79.52 \pm 4.4$ & $0.4334(2)$ & $4.639(1) \times 10^{38}$ \\
\hline 157 & 58725.954277635 & $563.7(5)$ & $4.53(4)$ & 0.2 & $62.35 \pm 5.0$ & $0.2824(2)$ & $3.024(2) \times 10^{38}$ \\
\hline 158 & 58725.954685243 & $566.0(5)$ & $4.03(6)$ & 0.17 & $20.724 \pm 0.2$ & $0.0835(1)$ & $8.943(9) \times 10^{37}$ \\
\hline 159 & 58725.954685303 & $570.0(7)$ & $6.71(8)$ & 0.4 & $39.63 \pm 3.9$ & $0.2659(3)$ & $2.847(3) \times 10^{38}$ \\
\hline 160 & 58725.955426311 & $554.5(5)$ & $4.31(6)$ & 0.16 & $7.088 \pm 0.1$ & $0.0306(0)$ & $3.272(3) \times 10^{37}$ \\
\hline 161 & 58725.955945044 & $566.0(5)$ & $5.08(7)$ & 0.165 & $15.97 \pm 0.1$ & $0.0811(1)$ & $8.678(9) \times 10^{37}$ \\
\hline 162 & 58725.956416807 & $564.6(4)$ & $7.85(3)$ & 0.4 & $147.11 \pm 4.0$ & $1.1548(1)$ & $1.236(0) \times 10^{39}$ \\
\hline 163 & 58725.956416815 & $566.0(5)$ & $8.32(9)$ & 0.38 & $43.303 \pm 0.4$ & $0.3602(4)$ & $3.856(4) \times 10^{38}$ \\
\hline 164 & 58725.956608320 & $567.0(5)$ & $3.47(4)$ & 0.1 & $62.63 \pm 5.5$ & $0.2173(2)$ & $2.326(2) \times 10^{38}$ \\
\hline 165 & 58725.956957750 & $566.0(5)$ & $3.39(5)$ & 0.2 & $10.926 \pm 0.1$ & $0.0370(0)$ & $3.962(4) \times 10^{37}$ \\
\hline 166 & 58725.957412467 & $565.8(5)$ & $4.11(6)$ & 0.11 & $5.241 \pm 0.1$ & $0.0215(0)$ & $2.304(2) \times 10^{37}$ \\
\hline 167 & 58725.958314159 & $569.0(7)$ & $5.89(5)$ & 0.15 & $58.68 \pm 4.0$ & $0.3456(2)$ & $3.700(2) \times 10^{38}$ \\
\hline 168 & 58725.959789238 & $569.9(8)$ & $5.6(6)$ & 0.15 & $46.34 \pm 4.4$ & $0.2595(3)$ & $2.778(2) \times 10^{38}$ \\
\hline 169 & 58725.959789241 & $566.0(5)$ & $4.99(7)$ & 0.2 & $12.981 \pm 0.1$ & $0.0647(1)$ & $6.931(7) \times 10^{37}$ \\
\hline 170 & 58725.961776128 & $570.0(12)$ & $10.3(9)$ & 0.1 & $31.69 \pm 3.1$ & $0.3264(4)$ & $3.494(4) \times 10^{38}$ \\
\hline 171 & 58725.962338775 & $566.0(5)$ & $2.04(3)$ & 0.17 & $13.112 \pm 0.1$ & $0.0268(0)$ & $2.867(2) \times 10^{37}$ \\
\hline 172 & 58725.962802477 & $569.4(5)$ & $9.62(9)$ & 0.21 & $16.386 \pm 0.1$ & $0.1577(2)$ & $1.688(1) \times 10^{38}$ \\
\hline 173 & 58725.962900390 & $565.0(12)$ & $7.3(9)$ & 0.2 & $24.58 \pm 3.6$ & $0.1794(5)$ & $1.921(4) \times 10^{38}$ \\
\hline 174 & 58725.964332786 & $565.9(7)$ & $9.14(4)$ & 0.2 & $100.11 \pm 3.5$ & $0.9150(1)$ & $9.795(1) \times 10^{38}$ \\
\hline 175 & 58725.965444014 & $568.9(8)$ & $3.53(4)$ & 0.1 & $50.48 \pm 5.0$ & $0.1782(2)$ & $1.908(2) \times 10^{38}$ \\
\hline 176 & 58725.966511348 & $564.6(3)$ & $2.55(1)$ & 0.3 & $247.36 \pm 6.3$ & $0.6308(1)$ & $6.752(0) \times 10^{38}$ \\
\hline 177 & 58725.968064539 & $565.9(5)$ & $4.31(4)$ & 0.2 & $65.65 \pm 4.7$ & $0.2829(2)$ & $3.029(1) \times 10^{38}$ \\
\hline 178 & 58725.970809777 & $566.1(4)$ & $4.89(6)$ & 0.1 & $45.12 \pm 4.5$ & $0.2206(3)$ & $2.362(2) \times 10^{38}$ \\
\hline 179 & 58725.973533113 & $566.0(5)$ & $4.06(6)$ & 0.2 & $18.017 \pm 0.1$ & $0.0732(1)$ & $7.834(7) \times 10^{37}$ \\
\hline 180 & 58725.973860175 & $563.2(5)$ & $2.01(3)$ & 0.18 & $16.979 \pm 0.1$ & $0.0341(0)$ & $3.649(3) \times 10^{37}$ \\
\hline 181 & 58725.973954948 & $564.6(5)$ & $3.17(3)$ & 0.1 & $80.2 \pm 5.6$ & $0.2542(1)$ & $2.722(1) \times 10^{38}$ \\
\hline 182 & 58725.973954961 & $566.0(5)$ & $5.33(8)$ & 0.135 & $15.892 \pm 0.1$ & $0.0846(1)$ & $9.061(8) \times 10^{37}$ \\
\hline 183 & 58725.974858771 & $561.0(5)$ & $4.19(6)$ & 0.06 & $9.786 \pm 0.1$ & $0.0410(0)$ & $4.390(3) \times 10^{37}$ \\
\hline
\end{tabular}


Table 1:

\begin{tabular}{|c|c|c|c|c|c|c|c|}
\hline Burst ID & $\begin{array}{c}\text { Burst time }^{a)} \\
\text { (MJD) }\end{array}$ & $\begin{array}{c}\mathrm{DM} \\
\left(\mathrm{pc} \mathrm{cm} \mathrm{cm}^{-3}\right)\end{array}$ & $\begin{array}{l}\text { Width } \\
\text { (ms) }\end{array}$ & $\begin{array}{c}\left.\text { Bandwidth }^{b}\right) \\
(\mathrm{GHz})\end{array}$ & $\begin{array}{c}\begin{array}{c}\text { Peak Flux } \\
(\mathrm{mJy})\end{array} \\
\end{array}$ & $\begin{array}{c}\text { Fluence } \\
\text { (Jy ms) }\end{array}$ & $\begin{array}{c}\text { Energy } \\
\text { (erg) }\end{array}$ \\
\hline 184 & 58725.976530224 & $566.0(5)$ & $3.21(5)$ & 0.135 & $26.036 \pm 0.2$ & $0.0837(1)$ & $8.955(7) \times 10^{37}$ \\
\hline 185 & 58725.976530252 & $566.5(3)$ & $3.14(3)$ & 0.05 & $82.9 \pm 5.7$ & $0.2603(1)$ & $2.787(1) \times 10^{38}$ \\
\hline 186 & 58725.977864314 & $566.0(5)$ & $4.03(6)$ & 0.2 & $13.086 \pm 0.1$ & $0.0527(0)$ & $5.640(4) \times 10^{37}$ \\
\hline 187 & 58725.978267731 & $566.0(5)$ & 3.77 ( 5 ) & 0.18 & $9.587 \pm 0.1$ & $0.0361(0)$ & $3.864(3) \times 10^{37}$ \\
\hline 188 & 58725.979701515 & $565.6(2)$ & $4.71(3)$ & 0.3 & $126.45 \pm 4.7$ & $0.5956(1)$ & $6.376(1) \times 10^{38}$ \\
\hline 189 & 58725.981471414 & $567.9(9)$ & $8.52(9)$ & 0.25 & $37.88 \pm 3.2$ & $0.3227(3)$ & $3.455(3) \times 10^{38}$ \\
\hline 191 & 58725.986480751 & $566.0(5)$ & $3.71(5)$ & 0.205 & $38.808 \pm 0.3$ & $0.1440(1)$ & $1.542(1) \times 10^{38}$ \\
\hline 192 & 58725.986480773 & $567.4(4)$ & $3.95(2)$ & 0.1 & $101.0 \pm 5.1$ & $0.3990(1)$ & $4.271(1) \times 10^{38}$ \\
\hline 193 & 58725.986508303 & $566.3(4)$ & $3.22(8)$ & 0.1 & $27.74 \pm 5.5$ & $0.0893(4)$ & $9.562(46) \times 10^{37}$ \\
\hline 194 & 58725.988479474 & $566.0(5)$ & $3.75(5)$ & 0.1 & $9.238 \pm 0.1$ & $0.0346(0)$ & $3.704(3) \times 10^{37}$ \\
\hline 195 & 58725.992262885 & $563.7(5)$ & $3.19(5)$ & 0.14 & $9.013 \pm 0.1$ & $0.0288(0)$ & $3.078(2) \times 10^{37}$ \\
\hline 196 & 58725.992599956 & $566.0(5)$ & $2.67(4)$ & 0.15 & $17.826 \pm 0.1$ & $0.0475(0)$ & $5.087(4) \times 10^{37}$ \\
\hline 197 & 58725.992788134 & $563.9(5)$ & $5.28(6)$ & 0.05 & $42.43 \pm 4.2$ & $0.2240(3)$ & $2.398(2) \times 10^{38}$ \\
\hline 198 & 58725.993465613 & $569.8(5)$ & $4.47(6)$ & 0.17 & $9.075 \pm 0.1$ & $0.0406(0)$ & $4.342(3) \times 10^{37}$ \\
\hline 200 & 58725.994952635 & $560.0(9)$ & $10.62(9)$ & 0.2 & $28.35 \pm 3.2$ & $0.3011(6)$ & $3.223(6) \times 10^{38}$ \\
\hline 201 & 58725.994952734 & $565.6(6)$ & $3.13(9)$ & 0.2 & $27.44 \pm 6.1$ & $0.0859(5)$ & $9.194(58) \times 10^{37}$ \\
\hline 202 & 58725.995131277 & $564.5(4)$ & $2.32(2)$ & 0.15 & $89.95 \pm 6.8$ & 0.2087 ( 1$)$ & $2.234(1) \times 10^{38}$ \\
\hline 203 & 58725.995131283 & $566.0(5)$ & $2.45(4)$ & 0.095 & $25.208 \pm 0.2$ & $0.0618(1)$ & $6.613(5) \times 10^{37}$ \\
\hline 204 & 58725.995459423 & $563.5(7)$ & $3.62(2)$ & 0.15 & $130.13 \pm 5.3$ & $0.4711(1)$ & $5.043(1) \times 10^{38}$ \\
\hline 205 & 58725.995459426 & $566.0(5)$ & $3.74(5)$ & 0.185 & $38.459 \pm 0.3$ & 0.1438 ( 1 ) & $1.540(1) \times 10^{38}$ \\
\hline 206 & 58725.995490050 & $566.0(5)$ & $5.51(8)$ & 0.2 & $13.982 \pm 0.1$ & $0.0770(1)$ & $8.242(6) \times 10^{37}$ \\
\hline 207 & 58725.997697326 & $569.8(5)$ & $8.53(9)$ & 0.19 & $7.698 \pm 0.1$ & $0.0657(1)$ & $7.028(5) \times 10^{37}$ \\
\hline 208 & 58725.998307767 & $570.0(8)$ & $5.52(6)$ & 0.1 & $47.5 \pm 4.3$ & $0.2622(2)$ & $2.807(2) \times 10^{38}$ \\
\hline 209 & 58726.918507224 & $564.6(3)$ & $2.6(2)$ & 0.2 & $142.99 \pm 7.3$ & $0.3718(1)$ & $3.980(1) \times 10^{38}$ \\
\hline 210 & 58726.919059259 & $562.8(5)$ & $3.36(5)$ & 0.15 & $11.909 \pm 0.1$ & $0.0400(1)$ & $4.284(5) \times 10^{37}$ \\
\hline 211 & 58726.921590032 & $568.9(5)$ & $7.53(9)$ & 0.17 & $8.304 \pm 0.1$ & $0.0625(1)$ & $6.690(9) \times 10^{37}$ \\
\hline 212 & 58726.922273689 & $568.5(5)$ & $7.39(9)$ & 0.12 & $8.057 \pm 0.1$ & $0.0595(1)$ & $6.373(8) \times 10^{37}$ \\
\hline 213 & 58726.924095985 & $566.3(5)$ & $4.45(6)$ & 0.33 & $12.601 \pm 0.1$ & 0.0561 ( 1$)$ & $6.000(7) \times 10^{37}$ \\
\hline 214 & 58726.924528635 & $566.0(5)$ & $3.32(5)$ & 0.045 & $13.896 \pm 0.2$ & 0.0461 ( 1 ) & $4.937(6) \times 10^{37}$ \\
\hline 215 & 58726.927726974 & $550.6(5)$ & $4.44(6)$ & 0.065 & $11.364 \pm 0.1$ & $0.0505(1)$ & $5.406(6) \times 10^{37}$ \\
\hline 218 & 58726.929464997 & $566.0(5)$ & $2.64(4)$ & 0.26 & $18.265 \pm 0.2$ & $0.0481(1)$ & $5.153(6) \times 10^{37}$ \\
\hline 219 & 58726.929640707 & $566.0(5)$ & $4.43(6)$ & 0.11 & $14.594 \pm 0.2$ & 0.0647 ( 1$)$ & $6.928(8) \times 10^{37}$ \\
\hline 220 & 58726.931862194 & $566.5(5)$ & $5.16(5)$ & 0.3 & $62.56 \pm 5.0$ & $0.3228(2)$ & $3.456(2) \times 10^{38}$ \\
\hline 221 & 58726.936039377 & $566.5(6)$ & $9.03(5)$ & 0.3 & $85.79 \pm 3.6$ & $0.7747(2)$ & $8.293(1) \times 10^{38}$ \\
\hline 222 & 58726.937128385 & $566.0(5)$ & $2.88(4)$ & 0.17 & $7.319 \pm 0.1$ & $0.0211(0)$ & $2.257(2) \times 10^{37}$ \\
\hline 223 & 58726.938300849 & $562.7(1)$ & $2.61(1)$ & 0.35 & $178.31 \pm 6.5$ & $0.4654(1)$ & $4.982(0) \times 10^{38}$ \\
\hline 224 & 58726.938300869 & $566.0(5)$ & $4.21(6)$ & 0.245 & $36.122 \pm 0.4$ & $0.1522(2)$ & $1.630(1) \times 10^{38}$ \\
\hline 225 & 58726.938411122 & $569.0(5)$ & $2.15(3)$ & 0.11 & $15.019 \pm 0.2$ & $0.0323(0)$ & $3.461(4) \times 10^{37}$ \\
\hline 226 & 58726.942553442 & $569.8(5)$ & $6.73(9)$ & 0.105 & $5.497 \pm 0.1$ & $0.0370(0)$ & $3.963(4) \times 10^{37}$ \\
\hline 227 & 58726.949717610 & $565.6(6)$ & 4.64 ( 6 ) & 0.05 & $46.32 \pm 4.9$ & $0.2149(3)$ & $2.301(3) \times 10^{38}$ \\
\hline 228 & 58726.954890369 & $564.1(4)$ & $1.82(2)$ & 0.2 & $80.45 \pm 7.7$ & $0.1464(2)$ & $1.567(1) \times 10^{38}$ \\
\hline 229 & 58726.954890386 & $566.0(5)$ & $2.47(4)$ & 0.205 & $13.946 \pm 0.1$ & $0.0344(0)$ & $3.682(3) \times 10^{37}$ \\
\hline 230 & 58726.957198400 & $566.0(5)$ & $4.06(6)$ & 0.205 & $19.506 \pm 0.2$ & $0.0791(1)$ & $8.472(8) \times 10^{37}$ \\
\hline 231 & 58726.957198413 & $567.4(7)$ & $3.98(3)$ & 0.13 & $79.38 \pm 5.0$ & $0.3159(1)$ & $3.382(1) \times 10^{38}$ \\
\hline 232 & 58726.957958985 & $566.0(5)$ & $4.31(6)$ & 0.395 & $89.553 \pm 0.8$ & $0.3856(4)$ & $4.127(4) \times 10^{38}$ \\
\hline 233 & 58726.957958996 & $564.6(3)$ & $3.76(1)$ & 0.35 & $407.9 \pm 5.2$ & $1.5337(0)$ & $1.642(0) \times 10^{39}$ \\
\hline 234 & 58726.959484347 & $566.0(5)$ & $6.89(9)$ & 0.21 & $13.581 \pm 0.1$ & $0.0935(1)$ & $1.001(0) \times 10^{38}$ \\
\hline 235 & 58726.959484423 & $566.9(12)$ & $6.86(6)$ & 0.2 & $53.11 \pm 3.9$ & $0.3643(2)$ & $3.900(2) \times 10^{38}$ \\
\hline 236 & 58726.960784931 & $563.0(4)$ & $5.95(6)$ & 0.1 & $44.68 \pm 4.1$ & $0.2658(3)$ & $2.846(2) \times 10^{38}$ \\
\hline 237 & 58726.960784945 & $563.7(5)$ & $3.51(5)$ & 0.1 & $12.409 \pm 0.1$ & $0.0435(0)$ & $4.662(4) \times 10^{37}$ \\
\hline 238 & 58726.965823587 & $566.2(4)$ & $3.01(4)$ & 0.1 & $51.1 \pm 5.9$ & $0.1538(2)$ & $1.647(2) \times 10^{38}$ \\
\hline 239 & 58726.967530175 & $566.0(5)$ & $2.6(4)$ & 0.07 & $17.151 \pm 0.1$ & $0.0446(0)$ & $4.775(4) \times 10^{37}$ \\
\hline 240 & 58726.968353199 & $567.9(8)$ & $5.23(4)$ & 0.3 & $70.34 \pm 4.5$ & $0.3679(2)$ & $3.938(1) \times 10^{38}$ \\
\hline 241 & 58726.969790874 & $569.0(5)$ & $7.68(9)$ & 0.19 & $20.1 \pm 0.2$ & 0.1544 ( 1$)$ & $1.653(1) \times 10^{38}$ \\
\hline 242 & 58726.969790915 & $565.6(7)$ & $8.13(8)$ & 0.4 & $44.51 \pm 3.6$ & $0.3619(3)$ & $3.874(3) \times 10^{38}$ \\
\hline 243 & 58726.971092351 & $566.0(5)$ & $2.78(4)$ & 0.12 & $15.221 \pm 0.1$ & $0.0424(0)$ & $4.537(4) \times 10^{37}$ \\
\hline 244 & 58726.972044638 & $568.5(5)$ & $2.12(3)$ & 0.07 & $19.645 \pm 0.2$ & $0.0417(0)$ & $4.466(4) \times 10^{37}$ \\
\hline 245 & 58726.972044650 & $563.7(7)$ & $3.25(5)$ & 0.05 & $44.9 \pm 5.5$ & 0.1459 ( 3 ) & $1.562(2) \times 10^{38}$ \\
\hline 246 & 58726.983188493 & $564.9(3)$ & $2.34(2)$ & 0.4 & $99.07 \pm 6.2$ & $0.2318(1)$ & $2.482(1) \times 10^{38}$ \\
\hline
\end{tabular}


Table 1:

\begin{tabular}{|c|c|c|c|c|c|c|c|}
\hline Burst ID & $\begin{array}{l}\text { Burst time }^{a)} \\
\text { (MJD) }\end{array}$ & $\begin{array}{c}\mathrm{DM} \\
\left(\mathrm{pc} \mathrm{cm}^{-3}\right)\end{array}$ & $\begin{array}{l}\text { Width } \\
\text { (ms) }\end{array}$ & $\begin{array}{c}\text { Bandwidth }^{b)} \\
(\mathrm{GHz})\end{array}$ & $\begin{array}{c}\text { Peak Flux } \\
(\mathrm{mJy})\end{array}$ & $\begin{array}{c}\begin{array}{c}\text { Fluence } \\
(\mathrm{Jy} \mathrm{ms})\end{array} \\
\end{array}$ & $\begin{array}{c}\text { Energy } \\
\text { (erg) }\end{array}$ \\
\hline 247 & 58726.983188495 & $566.0(5)$ & $2.81(4)$ & 0.31 & $21.764 \pm 0.2$ & $0.0611(1)$ & $6.543(5) \times 10^{37}$ \\
\hline 248 & 58726.987936974 & $564.5(3)$ & $3.15(2)$ & 0.4 & $104.95 \pm 5.6$ & $0.3306(1)$ & $3.539(1) \times 10^{38}$ \\
\hline 249 & 58726.987936984 & $566.0(5)$ & $3.8(6)$ & 0.2 & $17.794 \pm 0.1$ & $0.0676(1)$ & $7.234(6) \times 10^{37}$ \\
\hline 250 & 58726.989496877 & $570.0(11)$ & $8.28(9)$ & 0.12 & $34.02 \pm 3.5$ & $0.2817(3)$ & $3.015(3) \times 10^{38}$ \\
\hline 251 & 58726.995167647 & $567.8(8)$ & $6.77(8)$ & 0.05 & $38.88 \pm 3.7$ & $0.2632(3)$ & $2.818(3) \times 10^{38}$ \\
\hline 252 & 58726.996145324 & $568.5(5)$ & $3.74(5)$ & 0.195 & $4.141 \pm 0.0$ & $0.0155(0)$ & $1.656(1) \times 10^{37}$ \\
\hline 253 & 58726.998228733 & $569.5(12)$ & $8.97(8)$ & 0.05 & $44.45 \pm 3.2$ & 0.3987 ( 3 ) & $4.268(2) \times 10^{38}$ \\
\hline 254 & 58726.998228757 & $559.7(5)$ & $5.31(8)$ & 0.105 & $10.333 \pm 0.1$ & $0.0549(0)$ & $5.875(4) \times 10^{37}$ \\
\hline 255 & 58726.998228789 & $570.0(7)$ & $9.05(8)$ & 0.1 & $38.9 \pm 3.2$ & $0.3520(3)$ & $3.769(2) \times 10^{38}$ \\
\hline 256 & 58726.998595910 & $566.0(5)$ & $3.13(5)$ & 0.15 & $13.586 \pm 0.1$ & $0.0425(0)$ & $4.549(3) \times 10^{37}$ \\
\hline 257 & 58727.000199730 & $566.0(5)$ & $3.85(6)$ & 0.18 & $8.929 \pm 0.1$ & $0.0343(0)$ & $3.677(3) \times 10^{37}$ \\
\hline 258 & 58727.001202476 & $566.0(5)$ & $3.4(5)$ & 0.21 & $10.515 \pm 0.1$ & $0.0358(0)$ & $3.831(3) \times 10^{37}$ \\
\hline 259 & 58727.001219201 & $569.4(5)$ & $5.0(7)$ & 0.205 & $12.193 \pm 0.1$ & $0.0610(1)$ & $6.526(5) \times 10^{37}$ \\
\hline 260 & 58727.001430927 & $566.0(5)$ & $4.11(6)$ & 0.1 & $10.534 \pm 0.1$ & $0.0433(0)$ & $4.630(3) \times 10^{37}$ \\
\hline 261 & 58727.004049262 & $566.0(5)$ & $3.99(6)$ & 0.18 & $10.408 \pm 0.1$ & $0.0415(0)$ & $4.442(3) \times 10^{37}$ \\
\hline 262 & 58727.004170375 & $567.6(5)$ & $4.3(6)$ & 0.12 & $9.889 \pm 0.1$ & $0.0425(0)$ & $4.550(4) \times 10^{37}$ \\
\hline 263 & 58727.004234516 & $563.7(2)$ & $4.64(2)$ & 0.4 & $108.43 \pm 4.8$ & $0.5031(1)$ & $5.386(1) \times 10^{38}$ \\
\hline 264 & 58727.004234549 & $566.0(5)$ & $7.03(9)$ & 0.45 & $20.217 \pm 0.1$ & $0.1422(1)$ & $1.522(1) \times 10^{38}$ \\
\hline 265 & 58727.005230264 & $569.4(5)$ & $3.35(5)$ & 0.075 & $2.56 \pm 0.0$ & $0.0086(0)$ & $9.186(8) \times 10^{36}$ \\
\hline 266 & 58727.005347047 & $562.8(5)$ & $2.29(3)$ & 0.14 & $3.085 \pm 0.0$ & $0.0071(0)$ & $7.570(6) \times 10^{36}$ \\
\hline 267 & 58727.006365174 & $566.4(4)$ & $4.04(4)$ & 0.3 & $69.56 \pm 5.1$ & $0.2810(2)$ & $3.008(1) \times 10^{38}$ \\
\hline 268 & 58727.008108170 & $566.2(6)$ & $13.04(9)$ & 0.1 & $17.69 \pm 2.8$ & $0.2307(7)$ & $2.469(7) \times 10^{38}$ \\
\hline 269 & 58727.012203200 & $568.5(5)$ & $7.54(9)$ & 0.16 & $10.593 \pm 0.1$ & $0.0799(1)$ & $8.549(7) \times 10^{37}$ \\
\hline 270 & 58727.012321216 & $565.1(3)$ & $2.66(2)$ & 0.05 & $124.44 \pm 6.0$ & $0.3310(1)$ & $3.543(0) \times 10^{38}$ \\
\hline 271 & 58727.012986908 & $564.5(4)$ & $2.43(2)$ & 0.25 & $109.12 \pm 6.3$ & $0.2652(1)$ & $2.839(1) \times 10^{38}$ \\
\hline 272 & 58727.012986914 & $566.0(5)$ & $2.75(4)$ & 0.35 & $21.918 \pm 0.2$ & $0.0603(1)$ & $6.455(5) \times 10^{37}$ \\
\hline 273 & 58727.014227741 & $566.0(5)$ & $3.72(5)$ & 0.11 & $12.794 \pm 0.1$ & $0.0476(0)$ & $5.097(4) \times 10^{37}$ \\
\hline 274 & 58727.014655446 & $566.0(5)$ & 2.67 ( 4 ) & 0.12 & $10.779 \pm 0.1$ & $0.0288(0)$ & $3.084(2) \times 10^{37}$ \\
\hline 275 & 58727.017564085 & $565.9(5)$ & $3.63(4)$ & 0.1 & $52.6 \pm 5.2$ & $0.1909(2)$ & $2.044(2) \times 10^{38}$ \\
\hline 276 & 58727.018478524 & $566.0(5)$ & 3.57 ( 5 ) & 0.45 & $15.165 \pm 0.1$ & $0.0541(1)$ & $5.790(5) \times 10^{37}$ \\
\hline 277 & 58727.018478547 & 564.4 ( 3 ) & $3.2(3)$ & 0.15 & $81.62 \pm 5.5$ & $0.2612(1)$ & $2.796(1) \times 10^{38}$ \\
\hline 278 & 58727.019235532 & $568.5(5)$ & $5.17(7)$ & 0.15 & $6.379 \pm 0.1$ & $0.0329(0)$ & $3.527(3) \times 10^{37}$ \\
\hline 279 & 58727.020509855 & $564.2(3)$ & $10.39(8)$ & 0.1 & $48.07 \pm 3.1$ & $0.4995(2)$ & $5.347(2) \times 10^{38}$ \\
\hline 280 & 58727.023864654 & $565.7(6)$ & $4.45(7)$ & 0.1 & $52.22 \pm 4.7$ & $0.2324(3)$ & $2.488(3) \times 10^{38}$ \\
\hline 281 & 58727.023864719 & $565.8(5)$ & $4.37(9)$ & 0.1 & $36.56 \pm 4.8$ & $0.1598(5)$ & $1.710(5) \times 10^{38}$ \\
\hline 282 & 58727.026817440 & $565.7(3)$ & $4.75(2)$ & 0.35 & $109.44 \pm 4.7$ & $0.5198(1)$ & $5.565(1) \times 10^{38}$ \\
\hline 283 & 58727.026998230 & $566.0(5)$ & 3.98 ( 6 ) & 0.25 & $13.533 \pm 0.1$ & $0.0538(1)$ & $5.763(6) \times 10^{37}$ \\
\hline 284 & 58727.027891965 & $568.5(5)$ & $6.16(9)$ & 0.22 & $9.162 \pm 0.1$ & $0.0565(1)$ & $6.045(6) \times 10^{37}$ \\
\hline 285 & 58727.029127362 & $562.3(5)$ & $5.07(7)$ & 0.19 & $6.878 \pm 0.1$ & $0.0349(0)$ & $3.735(3) \times 10^{37}$ \\
\hline 286 & 58727.029613883 & $566.0(5)$ & $3.58(5)$ & 0.17 & $13.242 \pm 0.1$ & $0.0474(1)$ & $5.079(5) \times 10^{37}$ \\
\hline 287 & 58727.030106509 & $567.6(5)$ & $8.01(9)$ & 0.16 & $6.455 \pm 0.1$ & $0.0517(1)$ & $5.537(5) \times 10^{37}$ \\
\hline 288 & 58727.031383040 & $562.8(5)$ & $4.87(7)$ & 0.14 & $8.477 \pm 0.1$ & $0.0413(0)$ & $4.420(4) \times 10^{37}$ \\
\hline 289 & 58727.031993293 & $561.8(10)$ & 2.35 ( 3 ) & 0.1 & $73.5 \pm 6.3$ & $0.1727(2)$ & $1.849(1) \times 10^{38}$ \\
\hline 290 & 58727.031993302 & $566.0(5)$ & $2.57(4)$ & 0.15 & $17.21 \pm 0.2$ & $0.0442(0)$ & $4.734(5) \times 10^{37}$ \\
\hline 291 & 58727.035075514 & $568.5(5)$ & $5.28(8)$ & 0.12 & $10.055 \pm 0.1$ & $0.0531(1)$ & $5.683(6) \times 10^{37}$ \\
\hline 292 & 58727.035075572 & $568.6(5)$ & $6.73(8)$ & 0.1 & $40.16 \pm 4.1$ & $0.2703(3)$ & $2.893(3) \times 10^{38}$ \\
\hline 293 & 58727.036171631 & $566.2(4)$ & $4.18(5)$ & 0.1 & $55.87 \pm 5.3$ & $0.2335(2)$ & $2.500(2) \times 10^{38}$ \\
\hline 294 & 58727.039598375 & $564.4(6)$ & $11.05(8)$ & 0.1 & $52.06 \pm 3.2$ & $0.5753(3)$ & $6.158(2) \times 10^{38}$ \\
\hline 295 & 58727.043038447 & $565.0(5)$ & $5.2(8)$ & 0.1 & $6.006 \pm 0.1$ & $0.0312(0)$ & $3.343(4) \times 10^{37}$ \\
\hline 296 & 58727.043536157 & $564.5(5)$ & $6.54(9)$ & 0.11 & $8.691 \pm 0.1$ & $0.0568(1)$ & $6.083(7) \times 10^{37}$ \\
\hline 297 & 58727.044230758 & $566.0(5)$ & $2.1(3)$ & 0.15 & $19.689 \pm 0.2$ & $0.0413(0)$ & $4.425(5) \times 10^{37}$ \\
\hline 298 & 58727.048149883 & $567.6(5)$ & $7.52(9)$ & 0.155 & $13.767 \pm 0.1$ & $0.1036(1)$ & $1.109(1) \times 10^{38}$ \\
\hline 299 & 58727.048149906 & $564.3(2)$ & $7.63(7)$ & 0.15 & $50.71 \pm 4.1$ & $0.3869(3)$ & $4.142(3) \times 10^{38}$ \\
\hline 300 & 58727.051034577 & $564.8(4)$ & $2.98(4)$ & 0.2 & $57.89 \pm 6.7$ & $0.1725(3)$ & $1.847(3) \times 10^{38}$ \\
\hline 301 & 58727.054051542 & $568.1(5)$ & $3.51(5)$ & 0.12 & $7.29 \pm 0.1$ & $0.0256(0)$ & $2.740(3) \times 10^{37}$ \\
\hline 302 & 58727.058051523 & $566.3(5)$ & $4.25(6)$ & 0.205 & $14.161 \pm 0.2$ & $0.0602(1)$ & $6.445(8) \times 10^{37}$ \\
\hline 303 & 58727.058051531 & $567.2(5)$ & $5.91(7)$ & 0.3 & $54.54 \pm 5.0$ & $0.3223(3)$ & $3.451(3) \times 10^{38}$ \\
\hline 304 & 58727.062005534 & $564.0(3)$ & $3.13(3)$ & 0.3 & $84.97 \pm 6.7$ & $0.2660(2)$ & $2.847(2) \times 10^{38}$ \\
\hline 305 & 58727.066569033 & $565.1(5)$ & $4.46(3)$ & 0.2 & $102.01 \pm 5.9$ & $0.4550(2)$ & $4.870(1) \times 10^{38}$ \\
\hline 306 & 58727.072072188 & $566.3(5)$ & $7.72(9)$ & 0.175 & $7.202 \pm 0.1$ & $0.0556(1)$ & $5.955(6) \times 10^{37}$ \\
\hline 307 & 58727.075009419 & $566.7(5)$ & $5.57(8)$ & 0.205 & $12.492 \pm 0.1$ & $0.0696(1)$ & $7.450(8) \times 10^{37}$ \\
\hline 308 & 58727.077995899 & $563.2(5)$ & $5.23(8)$ & 0.03 & $8.703 \pm 0.1$ & $0.0455(1)$ & $4.876(5) \times 10^{37}$ \\
\hline 309 & 58727.080254242 & $567.2(5)$ & $6.71(9)$ & 0.45 & $10.518 \pm 0.1$ & $0.0706(1)$ & $7.553(9) \times 10^{37}$ \\
\hline
\end{tabular}


Table 1:

\begin{tabular}{|c|c|c|c|c|c|c|c|}
\hline Burst ID & $\begin{array}{l}\text { Burst time }^{a)} \\
\text { (MJD) }\end{array}$ & $\begin{array}{c}\mathrm{DM} \\
\left(\mathrm{pc} \mathrm{cm}^{-3}\right)\end{array}$ & $\begin{array}{l}\text { Width } \\
\text { (ms) }\end{array}$ & $\begin{array}{c}\text { Bandwidth }^{b)} \\
(\mathrm{GHz})\end{array}$ & $\begin{array}{c}\text { Peak Flux } \\
(\mathrm{mJy})\end{array}$ & $\begin{array}{c}\begin{array}{c}\text { Fluence } \\
(\mathrm{Jy} \mathrm{ms})\end{array} \\
\end{array}$ & $\begin{array}{c}\text { Energy } \\
\text { (erg) }\end{array}$ \\
\hline 310 & 58727.087527934 & $565.1(3)$ & $5.98(9)$ & 0.4 & $51.88 \pm 6.5$ & $0.3102(8)$ & $3.321(8) \times 10^{38}$ \\
\hline 311 & 58727.087528001 & $566.0(5)$ & $4.41(6)$ & 0.12 & $57.355 \pm 0.7$ & $0.2532(4)$ & $2.711(3) \times 10^{38}$ \\
\hline 312 & 58727.087870348 & $564.5(5)$ & $6.56(9)$ & 0.15 & $14.166 \pm 0.2$ & $0.0929(1)$ & $9.944(14) \times 10^{37}$ \\
\hline 313 & 58727.092331852 & $565.8(7)$ & $10.94(9)$ & 0.4 & $47.37 \pm 4.5$ & $0.5182(6)$ & $5.548(5) \times 10^{38}$ \\
\hline 314 & 58727.096620708 & $564.5(5)$ & $6.58(9)$ & 0.045 & $12.064 \pm 0.2$ & $0.0793(1)$ & $8.491(13) \times 10^{37}$ \\
\hline 315 & 58727.096620741 & $568.9(6)$ & $5.63(7)$ & 0.2 & $59.98 \pm 6.3$ & $0.3377(5)$ & $3.615(4) \times 10^{38}$ \\
\hline 316 & 58727.097070964 & $567.6(5)$ & $8.82(9)$ & 0.165 & $8.75 \pm 0.1$ & $0.0772(1)$ & $8.263(13) \times 10^{37}$ \\
\hline 317 & 58727.099640113 & $564.3(6)$ & $6.16(4)$ & 0.15 & $98.17 \pm 6.2$ & $0.6047(3)$ & $6.474(2) \times 10^{38}$ \\
\hline 318 & 58727.099640136 & $566.0(5)$ & $6.68(9)$ & 0.145 & $25.828 \pm 0.4$ & $0.1724(3)$ & $1.846(3) \times 10^{38}$ \\
\hline 319 & 58727.894486748 & $563.2(5)$ & $4.15(6)$ & 0.17 & $9.272 \pm 0.1$ & $0.0385(1)$ & $4.119(5) \times 10^{37}$ \\
\hline 320 & 58727.903804424 & $566.0(5)$ & $4.04(6)$ & 0.46 & $98.872 \pm 1.0$ & $0.3996(5)$ & $4.278(4) \times 10^{38}$ \\
\hline 321 & 58727.906568088 & $565.0(5)$ & $4.75(7)$ & 0.14 & $6.057 \pm 0.1$ & $0.0288(0)$ & $3.081(3) \times 10^{37}$ \\
\hline 322 & 58727.908047227 & $565.9(5)$ & $7.14(9)$ & 0.17 & $6.144 \pm 0.1$ & $0.0439(1)$ & $4.698(6) \times 10^{37}$ \\
\hline 323 & 58727.910692196 & $566.0(5)$ & $3.55(5)$ & 0.2 & $32.362 \pm 0.4$ & $0.1150(2)$ & $1.231(1) \times 10^{38}$ \\
\hline 324 & 58727.910692207 & $566.7(3)$ & $3.46(2)$ & 0.2 & $149.2 \pm 6.7$ & $0.5162(1)$ & $5.526(1) \times 10^{38}$ \\
\hline 325 & 58727.911545632 & $567.2(5)$ & $2.62(4)$ & 0.11 & $4.829 \pm 0.1$ & $0.0127(0)$ & $1.356(1) \times 10^{37}$ \\
\hline 326 & 58727.915270908 & $567.2(5)$ & $8.12(9)$ & 0.09 & $4.804 \pm 0.1$ & $0.0390(1)$ & $4.175(5) \times 10^{37}$ \\
\hline 327 & 58727.915750478 & $563.8(2)$ & $2.81(0)$ & 0.3 & $595.3 \pm 7.1$ & $1.6728(0)$ & $1.791(0) \times 10^{39}$ \\
\hline 328 & 58727.918912967 & $566.0(5)$ & $3.1(5)$ & 0.205 & $46.053 \pm 0.5$ & 0.1427 ( 2) & $1.527(2) \times 10^{38}$ \\
\hline 329 & 58727.918912974 & $565.9(3)$ & 3.15 ( 1) & 0.25 & $178.59 \pm 6.6$ & $0.5626(1)$ & $6.022(0) \times 10^{38}$ \\
\hline 330 & 58727.921086782 & $567.2(5)$ & $3.34(5)$ & 0.165 & $9.122 \pm 0.1$ & $0.0305(0)$ & $3.265(4) \times 10^{37}$ \\
\hline 331 & 58727.921497784 & $568.5(5)$ & $7.31(9)$ & 0.27 & $11.145 \pm 0.1$ & $0.0815(1)$ & $8.723(11) \times 10^{37}$ \\
\hline 332 & 58727.921793235 & $566.0(5)$ & $3.83(6)$ & 0.19 & $36.875 \pm 0.4$ & $0.1411(2)$ & $1.511(1) \times 10^{38}$ \\
\hline 333 & 58727.922446544 & $566.3(5)$ & $4.84(7)$ & 0.4 & $14.873 \pm 0.2$ & $0.0720(1)$ & $7.710(10) \times 10^{37}$ \\
\hline 334 & 58727.922446564 & $566.1(7)$ & $7.42(6)$ & 0.05 & $61.72 \pm 4.4$ & $0.4580(3)$ & $4.902(3) \times 10^{38}$ \\
\hline 335 & 58727.923685152 & $563.2(5)$ & 4.04 ( 6 ) & 0.24 & $17.38 \pm 0.2$ & $0.0703(1)$ & $7.521(9) \times 10^{37}$ \\
\hline 336 & 58727.923993102 & $566.0(5)$ & 3.04 ( 4 ) & 0.255 & $20.589 \pm 0.2$ & $0.0626(1)$ & $6.698(8) \times 10^{37}$ \\
\hline 337 & 58727.923993118 & $565.8(6)$ & $3.28(4)$ & 0.05 & $65.66 \pm 6.5$ & $0.2154(2)$ & $2.305(2) \times 10^{38}$ \\
\hline 338 & 58727.925970166 & $561.0(5)$ & $4.0(6)$ & 0.17 & $7.464 \pm 0.1$ & $0.0298(0)$ & $3.194(4) \times 10^{37}$ \\
\hline 339 & 58727.928556763 & $562.8(2)$ & 3.78 ( 3 ) & 0.1 & $92.52 \pm 5.5$ & $0.3497(1)$ & $3.744(1) \times 10^{38}$ \\
\hline 340 & 58727.938197202 & $566.0(5)$ & $4.55(7)$ & 0.175 & $12.284 \pm 0.1$ & $0.0559(1)$ & $5.982(6) \times 10^{37}$ \\
\hline 341 & 58727.940379082 & $566.0(5)$ & $3.7(5)$ & 0.2 & $25.45 \pm 0.2$ & $0.0942(1)$ & $1.008(1) \times 10^{38}$ \\
\hline 342 & 58727.940379098 & $567.0(3)$ & $4.36(3)$ & 0.1 & $100.11 \pm 4.9$ & $0.4365(1)$ & $4.672(1) \times 10^{38}$ \\
\hline 343 & 58727.945932552 & $564.5(5)$ & $8.95(9)$ & 0.07 & $7.819 \pm 0.1$ & $0.0700(1)$ & $7.494(8) \times 10^{37}$ \\
\hline 344 & 58727.948012392 & $566.0(5)$ & $2.73(4)$ & 0.36 & $226.473 \pm 2.1$ & $0.6185(7)$ & $6.621(7) \times 10^{38}$ \\
\hline 345 & 58727.949899198 & $564.5(5)$ & $5.3(8)$ & 0.4 & $18.051 \pm 0.2$ & $0.0957(1)$ & $1.025(1) \times 10^{38}$ \\
\hline 346 & 58727.950393163 & $566.0(5)$ & $3.05(4)$ & 0.165 & $17.19 \pm 0.2$ & $0.0524(1)$ & $5.614(5) \times 10^{37}$ \\
\hline 347 & 58727.953778656 & $564.5(5)$ & $3.15(5)$ & 0.102 & $7.222 \pm 0.1$ & $0.0227(0)$ & $2.432(2) \times 10^{37}$ \\
\hline 348 & 58727.962349377 & $566.0(5)$ & $4.1(6)$ & 0.133 & $15.512 \pm 0.1$ & $0.0636(1)$ & $6.809(6) \times 10^{37}$ \\
\hline 349 & 58727.962709384 & $569.0(5)$ & 4.03 ( 6 ) & 0.105 & $14.443 \pm 0.1$ & $0.0582(1)$ & $6.235(5) \times 10^{37}$ \\
\hline 350 & 58727.962766806 & $567.6(5)$ & $5.46(8)$ & 0.11 & $9.888 \pm 0.1$ & $0.0540(1)$ & $5.780(5) \times 10^{37}$ \\
\hline 351 & 58727.963493087 & $566.0(5)$ & 4.04 ( 6 ) & 0.27 & $12.365 \pm 0.1$ & $0.0500(0)$ & $5.348(5) \times 10^{37}$ \\
\hline 352 & 58727.963965689 & $566.0(5)$ & 3.13 ( 5 ) & 0.21 & $12.591 \pm 0.1$ & $0.0394(0)$ & $4.213(3) \times 10^{37}$ \\
\hline 353 & 58727.964838323 & $552.4(5)$ & $7.35(9)$ & 0.175 & $4.82 \pm 0.0$ & $0.0354(0)$ & $3.791(3) \times 10^{37}$ \\
\hline 354 & 58727.965134923 & $566.0(5)$ & $4.37(6)$ & 0.13 & $10.409 \pm 0.1$ & $0.0455(0)$ & $4.869(4) \times 10^{37}$ \\
\hline 355 & 58727.970677638 & $562.8(2)$ & 4.89 ( 3 ) & 0.4 & $120.39 \pm 4.5$ & $0.5887(1)$ & $6.302(1) \times 10^{38}$ \\
\hline 356 & 58727.970677717 & $562.5(2)$ & $4.76(3)$ & 0.4 & $123.4 \pm 4.6$ & $0.5874(2)$ & $6.288(1) \times 10^{38}$ \\
\hline 357 & 58727.970677788 & $566.0(5)$ & $9.84(9)$ & 0.37 & $21.424 \pm 0.2$ & $0.2108(2)$ & $2.257(2) \times 10^{38}$ \\
\hline 358 & 58727.970677816 & $563.7(1)$ & $6.96(7)$ & 0.4 & $58.35 \pm 3.9$ & $0.4061(3)$ & $4.347(2) \times 10^{38}$ \\
\hline 359 & 58727.971658543 & $565.5(5)$ & $3.86(4)$ & 0.2 & $65.39 \pm 5.0$ & $0.2524(2)$ & $2.702(1) \times 10^{38}$ \\
\hline 360 & 58727.973011852 & $564.8(3)$ & $4.02(3)$ & 0.2 & $82.7 \pm 4.8$ & $0.3325(1)$ & $3.559(1) \times 10^{38}$ \\
\hline 361 & 58727.976479774 & $568.5(5)$ & $6.92(9)$ & 0.3 & $13.19 \pm 0.1$ & $0.0912(1)$ & $9.764(8) \times 10^{37}$ \\
\hline 362 & 58727.979717135 & $566.0(5)$ & $6.01(9)$ & 0.23 & $17.25 \pm 0.1$ & $0.1036(1)$ & $1.109(0) \times 10^{38}$ \\
\hline 363 & 58727.981083444 & $568.5(5)$ & $6.51(9)$ & 0.23 & $8.493 \pm 0.1$ & $0.0553(0)$ & $5.915(4) \times 10^{37}$ \\
\hline 364 & 58727.981978004 & $566.3(5)$ & $6.34(9)$ & 0.16 & $6.883 \pm 0.1$ & $0.0437(0)$ & $4.673(3) \times 10^{37}$ \\
\hline 365 & 58727.982031236 & $566.0(5)$ & $3.88(6)$ & 0.4 & $23.014 \pm 0.2$ & $0.0894(1)$ & $9.565(8) \times 10^{37}$ \\
\hline 366 & 58727.984559171 & $566.0(5)$ & 3.94 ( 6 ) & 0.17 & $14.551 \pm 0.1$ & $0.0574(0)$ & $6.144(5) \times 10^{37}$ \\
\hline 367 & 58727.986802065 & $566.0(5)$ & $2.66(4)$ & 0.25 & $7.795 \pm 0.1$ & $0.0207(0)$ & $2.216(1) \times 10^{37}$ \\
\hline 368 & 58727.989624890 & $565.5(5)$ & 6.47 ( 1 ) & 0.35 & $705.04 \pm 6.7$ & $4.5616(0)$ & $4.883(0) \times 10^{39}$ \\
\hline 369 & 58727.994825462 & $566.0(5)$ & $3.29(5)$ & 0.29 & $9.108 \pm 0.1$ & $0.0299(0)$ & $3.204(2) \times 10^{37}$ \\
\hline 370 & 58727.996943109 & $566.7(5)$ & $6.47(9)$ & 0.13 & $4.98 \pm 0.0$ & $0.0322(0)$ & $3.452(2) \times 10^{37}$ \\
\hline 371 & 58727.997917937 & $569.4(7)$ & $11.03(9)$ & 0.05 & $27.66 \pm 3.0$ & $0.3051(5)$ & $3.266(4) \times 10^{38}$ \\
\hline 372 & 58727.999875050 & $565.6(4)$ & $3.5(5)$ & 0.05 & $48.82 \pm 5.4$ & $0.1709(2)$ & $1.829(2) \times 10^{38}$ \\
\hline
\end{tabular}


Table 1:

\begin{tabular}{|c|c|c|c|c|c|c|c|}
\hline Burst ID & $\begin{array}{c}\text { Burst time }^{a)} \\
\text { (MJD) }\end{array}$ & 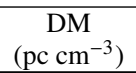 & $\begin{array}{l}\text { Width } \\
\text { (ms) }\end{array}$ & $\begin{array}{c}\left.\text { Bandwidth }^{b}\right) \\
(\mathrm{GHz})\end{array}$ & $\begin{array}{c}\begin{array}{c}\text { Peak Flux } \\
(\mathrm{mJy})\end{array} \\
\end{array}$ & $\begin{array}{c}\text { Fluence } \\
\text { (Jy ms) }\end{array}$ & $\begin{array}{c}\text { Energy } \\
\text { (erg) }\end{array}$ \\
\hline 373 & 58728.001683903 & $567.2(4)$ & $4.18(2)$ & 0.4 & $94.36 \pm 4.7$ & 0.3944 ( 1) & $4.222(1) \times 10^{38}$ \\
\hline 374 & 58728.001741661 & $562.2(1)$ & $1.81(3)$ & 0.4 & $74.3 \pm 10.6$ & $0.1345(3)$ & $1.440(3) \times 10^{38}$ \\
\hline 375 & 58728.001741701 & $562.1(1)$ & $4.69(3)$ & 0.3 & $153.4 \pm 4.6$ & $0.7195(1)$ & $7.702(1) \times 10^{38}$ \\
\hline 376 & 58728.002466245 & $566.1(3)$ & $3.38(5)$ & 0.2 & $48.17 \pm 5.3$ & $0.1628(2)$ & $1.743(2) \times 10^{38}$ \\
\hline 377 & 58728.005099678 & $566.5(6)$ & $16.57(9)$ & 0.25 & $39.37 \pm 2.5$ & $0.6524(3)$ & $6.983(3) \times 10^{38}$ \\
\hline 378 & 58728.006869155 & $562.6(2)$ & $5.93(3)$ & 0.4 & $107.36 \pm 4.0$ & $0.6366(1)$ & $6.815(1) \times 10^{38}$ \\
\hline 380 & 58728.014628541 & $569.0(5)$ & $5.42(8)$ & 0.125 & $4.573 \pm 0.0$ & $0.0248(0)$ & $2.654(2) \times 10^{37}$ \\
\hline 381 & 58728.014753972 & $565.5(5)$ & $4.06(4)$ & 0.1 & $53.74 \pm 4.9$ & $0.2182(2)$ & $2.336(2) \times 10^{38}$ \\
\hline 382 & 58728.014754022 & $564.1(5)$ & $4.72(7)$ & 0.105 & $15.846 \pm 0.1$ & $0.0748(1)$ & $8.007(7) \times 10^{37}$ \\
\hline 383 & 58728.014754076 & $566.5(3)$ & $2.68(5)$ & 0.2 & $38.91 \pm 5.9$ & $0.1043(3)$ & $1.116(3) \times 10^{38}$ \\
\hline 384 & 58728.015102060 & $564.5(5)$ & $7.98(9)$ & 0.04 & $5.438 \pm 0.1$ & $0.0434(0)$ & $4.645(4) \times 10^{37}$ \\
\hline 385 & 58728.020969046 & $565.1(5)$ & $3.71(0)$ & 0.2 & $711.8 \pm 6.0$ & $2.6408(0)$ & $2.827(0) \times 10^{39}$ \\
\hline 386 & 58728.021642554 & $566.0(5)$ & $2.81(4)$ & 0.09 & $10.477 \pm 0.1$ & $0.0294(0)$ & $3.151(3) \times 10^{37}$ \\
\hline 387 & 58728.022013163 & $569.8(5)$ & $5.72(8)$ & 0.105 & $8.875 \pm 0.1$ & $0.0508(1)$ & $5.437(5) \times 10^{37}$ \\
\hline 389 & 58728.025301656 & $566.0(5)$ & $6.89(9)$ & 0.05 & $11.433 \pm 0.1$ & 0.0787 ( 1$)$ & $8.430(8) \times 10^{37}$ \\
\hline 390 & 58728.025301706 & $563.2(4)$ & $6.69(6)$ & 0.2 & $50.73 \pm 4.0$ & $0.3394(2)$ & $3.633(2) \times 10^{38}$ \\
\hline 391 & 58728.025749614 & $565.6(6)$ & $10.2(9)$ & 0.1 & $23.84 \pm 3.2$ & $0.2432(5)$ & $2.603(5) \times 10^{38}$ \\
\hline 392 & 58728.026909373 & $558.9(5)$ & $8.52(9)$ & 0.4 & $1.69 \pm 0.0$ & $0.0144(0)$ & $1.541(1) \times 10^{37}$ \\
\hline 393 & 58728.030213995 & $564.4(3)$ & $2.82(1)$ & 0.35 & $376.01 \pm 5.9$ & $1.0603(0)$ & $1.135(0) \times 10^{39}$ \\
\hline 394 & 58728.030214000 & $566.0(5)$ & $2.83(4)$ & 0.105 & $91.667 \pm 0.9$ & $0.2599(3)$ & $2.782(3) \times 10^{38}$ \\
\hline 395 & 58728.031672211 & $566.0(5)$ & $3.17(5)$ & 0.205 & $5.227 \pm 0.1$ & $0.0166(0)$ & $1.774(1) \times 10^{37}$ \\
\hline 396 & 58728.044366594 & $569.0(5)$ & $4.09(6)$ & 0.26 & $9.115 \pm 0.1$ & $0.0373(0)$ & $3.994(4) \times 10^{37}$ \\
\hline 397 & 58728.044896019 & $566.0(5)$ & $4.16(6)$ & 0.13 & $17.5 \pm 0.2$ & $0.0728(1)$ & $7.793(9) \times 10^{37}$ \\
\hline 398 & 58728.052382221 & $566.0(5)$ & $2.9(4)$ & 0.3 & $12.698 \pm 0.1$ & $0.0369(0)$ & $3.948(5) \times 10^{37}$ \\
\hline 399 & 58728.057160920 & $564.5(5)$ & $7.63(9)$ & 0.4 & $11.771 \pm 0.1$ & $0.0898(1)$ & $9.612(13) \times 10^{37}$ \\
\hline 400 & 58728.057307835 & $570.0(4)$ & $9.26(4)$ & 0.25 & $103.63 \pm 3.9$ & $0.9596(2)$ & $1.027(0) \times 10^{39}$ \\
\hline 401 & 58728.059427792 & $564.0(4)$ & $5.01(1)$ & 0.4 & $399.1 \pm 6.2$ & 1.9995 ( 1 ) & $2.140(0) \times 10^{39}$ \\
\hline 402 & 58728.059427793 & $566.0(5)$ & $5.28(8)$ & 0.05 & $102.196 \pm 1.2$ & $0.5392(7)$ & $5.772(7) \times 10^{38}$ \\
\hline 403 & 58728.067769481 & $566.0(5)$ & $2.08(3)$ & 0.04 & $27.758 \pm 0.4$ & 0.0577 ( 1) & $6.177(9) \times 10^{37}$ \\
\hline 404 & 58728.067769482 & $565.3(3)$ & $1.78(2)$ & 0.2 & $131.45 \pm 9.9$ & $0.2340(1)$ & $2.505(1) \times 10^{38}$ \\
\hline 407 & 58728.075077042 & $566.0(5)$ & $2.88(4)$ & 0.39 & $193.721 \pm 2.0$ & $0.5582(7)$ & $5.975(7) \times 10^{38}$ \\
\hline 408 & 58728.076347404 & $566.4(5)$ & $3.23(2)$ & 0.4 & $123.68 \pm 7.5$ & $0.3995(2)$ & $4.276(1) \times 10^{38}$ \\
\hline 409 & 58728.077734777 & $567.0(8)$ & $3.53(3)$ & 0.2 & $96.54 \pm 6.8$ & $0.3408(2)$ & $3.648(2) \times 10^{38}$ \\
\hline 410 & 58728.958907809 & $566.0(5)$ & $3.46(5)$ & 0.26 & $6.847 \pm 0.1$ & $0.0237(0)$ & $2.538(2) \times 10^{37}$ \\
\hline 411 & 58728.965670285 & $566.5(5)$ & $3.33(2)$ & 0.15 & $115.02 \pm 5.7$ & $0.3830(1)$ & $4.100(1) \times 10^{38}$ \\
\hline 412 & 58728.968898361 & $569.4(8)$ & $5.32(2)$ & 0.2 & $112.07 \pm 4.3$ & $0.5962(1)$ & $6.382(1) \times 10^{38}$ \\
\hline 413 & 58728.975048150 & $565.1(6)$ & $2.13(2)$ & 0.05 & $93.12 \pm 6.8$ & $0.1983(1)$ & $2.123(1) \times 10^{38}$ \\
\hline 414 & 58728.983983731 & $564.6(3)$ & $2.31(1)$ & 0.3 & $333.07 \pm 6.4$ & $0.7694(0)$ & $8.236(0) \times 10^{38}$ \\
\hline 415 & 58728.984960173 & $564.3(3)$ & $2.69(2)$ & 0.05 & $82.37 \pm 5.9$ & $0.2216(1)$ & $2.372(1) \times 10^{38}$ \\
\hline 416 & 58728.988427881 & $567.2(6)$ & $6.04(3)$ & 0.15 & $87.38 \pm 4.1$ & $0.5278(1)$ & $5.650(1) \times 10^{38}$ \\
\hline 417 & 58728.993927624 & $566.0(3)$ & $3.24(1)$ & 0.2 & $187.03 \pm 5.3$ & $0.6060(1)$ & $6.487(0) \times 10^{38}$ \\
\hline 418 & 58729.002729388 & $562.5(4)$ & $1.74(2)$ & 0.05 & $81.42 \pm 7.6$ & 0.1417 ( 1 ) & $1.517(1) \times 10^{38}$ \\
\hline 419 & 58729.003209837 & $567.2(4)$ & $4.04(5)$ & 0.05 & $48.92 \pm 5.0$ & $0.1976(2)$ & $2.116(2) \times 10^{38}$ \\
\hline 420 & 58729.004947698 & $568.3(7)$ & $4.41(4)$ & 0.40 & $64.7 \pm 4.7$ & $0.2853(2)$ & $3.054(1) \times 10^{38}$ \\
\hline 421 & 58729.006687544 & $566.4(2)$ & $2.53(2)$ & 0.15 & $75.04 \pm 6.0$ & 0.1898 ( 1 ) & $2.032(1) \times 10^{38}$ \\
\hline 422 & 58729.008514024 & $569.0(8)$ & $6.05(8)$ & 0.05 & $36.38 \pm 4.0$ & $0.2201(3)$ & $2.356(3) \times 10^{38}$ \\
\hline 423 & 58729.009406471 & $568.0(5)$ & $5.77(6)$ & 0.15 & $47.36 \pm 4.2$ & $0.2733(2)$ & $2.925(2) \times 10^{38}$ \\
\hline 424 & 58729.014642331 & $566.0(5)$ & $4.43(7)$ & 0.15 & $35.21 \pm 4.8$ & $0.1560(3)$ & $1.670(3) \times 10^{38}$ \\
\hline 425 & 58729.014642592 & $568.0(7)$ & $4.05(5)$ & 0.15 & $51.07 \pm 5.0$ & $0.2068(2)$ & $2.214(2) \times 10^{38}$ \\
\hline 426 & 58729.014642996 & $569.8(8)$ & $6.95(9)$ & 0.15 & $29.39 \pm 4.1$ & $0.2043(5)$ & $2.187(5) \times 10^{38}$ \\
\hline 427 & 58729.014993412 & $567.0(6)$ & $5.43(6)$ & 0.4 & $44.38 \pm 4.5$ & $0.2410(3)$ & $2.580(3) \times 10^{38}$ \\
\hline 428 & 58729.015242789 & $569.8(5)$ & $7.59(9)$ & 0.16 & $7.514 \pm 0.1$ & $0.0570(1)$ & $6.104(6) \times 10^{37}$ \\
\hline 429 & 58729.015584209 & $564.2(4)$ & $2.88(1)$ & 0.4 & $323.67 \pm 5.7$ & $0.9322(0)$ & $9.979(0) \times 10^{38}$ \\
\hline 430 & 58729.015584262 & $564.4(4)$ & $3.51(3)$ & 0.4 & $87.36 \pm 5.3$ & $0.3066(2)$ & $3.282(1) \times 10^{38}$ \\
\hline 431 & 58729.017058848 & $564.6(4)$ & $2.02(2)$ & 0.05 & $86.7 \pm 6.8$ & $0.1751(1)$ & $1.875(1) \times 10^{38}$ \\
\hline 432 & 58729.018066356 & $564.7(5)$ & $2.88(0)$ & 0.4 & $10.1 \pm 2.9$ & $0.0291(7)$ & $3.114(19) \times 10^{37}$ \\
\hline 433 & 58729.018066385 & $565.6(5)$ & $4.27(3)$ & 0.4 & $647.47 \pm 38.9$ & $2.7647(11)$ & $2.960(1) \times 10^{39}$ \\
\hline 434 & 58729.018066512 & $563.8(5)$ & 4.89 (1) & 0.4 & $381.4 \pm 6.0$ & $1.8651(1)$ & $1.997(0) \times 10^{39}$ \\
\hline 435 & 58729.018066596 & $563.8(5)$ & $4.71(2)$ & 0.4 & $218.79 \pm 6.1$ & $1.0305(1)$ & $1.103(0) \times 10^{39}$ \\
\hline
\end{tabular}


Table 1:

\begin{tabular}{|c|c|c|c|c|c|c|c|}
\hline Burst ID & $\begin{array}{c}\text { Burst time }^{a)} \\
\text { (MJD) }\end{array}$ & $\begin{array}{c}\mathrm{DM} \\
(\mathrm{pc} \mathrm{cm}-3)\end{array}$ & $\begin{array}{l}\text { Width } \\
\text { (ms) }\end{array}$ & $\begin{array}{c}\left.\text { Bandwidth }^{b}\right) \\
(\mathrm{GHz})\end{array}$ & $\begin{array}{c}\begin{array}{c}\text { Peak Flux } \\
(\mathrm{mJy})\end{array} \\
\end{array}$ & $\begin{array}{c}\text { Fluence } \\
\text { (Jy ms) }\end{array}$ & $\begin{array}{c}\text { Energy } \\
\text { (erg) }\end{array}$ \\
\hline 436 & 58729.018204959 & $565.3(4)$ & $4.5(5)$ & 0.4 & $50.7 \pm 4.8$ & $0.2281(2)$ & $2.442(2) \times 10^{38}$ \\
\hline 437 & 58729.018378565 & $564.9(2)$ & $3.0(0)$ & 0.25 & $754.67 \pm 5.8$ & $2.2640(0)$ & $2.424(0) \times 10^{39}$ \\
\hline 438 & 58729.018476194 & $563.1(2)$ & $2.2(1)$ & 0.2 & $161.56 \pm 6.9$ & $0.3554(1)$ & $3.805(0) \times 10^{38}$ \\
\hline 439 & 58729.018646540 & $562.9(6)$ & $9.87(7)$ & 0.1 & $51.12 \pm 3.2$ & $0.5046(2)$ & $5.401(2) \times 10^{38}$ \\
\hline 440 & 58729.019532739 & $563.6(4)$ & $2.02(1)$ & 0.4 & $277.29 \pm 7.3$ & $0.5601(0)$ & $5.996(0) \times 10^{38}$ \\
\hline 441 & 58729.023094566 & $569.0(5)$ & $6.54(9)$ & 0.205 & $8.057 \pm 0.1$ & $0.0527(1)$ & $5.643(6) \times 10^{37}$ \\
\hline 443 & 58729.023957615 & $565.4(4)$ & $3.15(3)$ & 0.1 & $74.67 \pm 5.9$ & $0.2352(2)$ & $2.518(1) \times 10^{38}$ \\
\hline 444 & 58729.024756254 & $569.6(7)$ & $6.85(7)$ & 0.1 & $43.51 \pm 3.9$ & $0.2980(3)$ & $3.191(3) \times 10^{38}$ \\
\hline 445 & 58729.027076819 & $565.1(6)$ & $5.27(6)$ & 0.15 & $47.04 \pm 4.4$ & $0.2479(3)$ & $2.654(2) \times 10^{38}$ \\
\hline 446 & 58729.030253301 & $566.3(3)$ & $2.31(2)$ & 0.2 & $96.78 \pm 6.5$ & $0.2236(1)$ & $2.393(1) \times 10^{38}$ \\
\hline 447 & 58729.030253357 & $566.8(3)$ & $4.28(1)$ & 0.3 & $208.79 \pm 4.9$ & $0.8936(1)$ & $9.566(0) \times 10^{38}$ \\
\hline 448 & 58729.031064914 & $566.7(4)$ & $5.46(4)$ & 0.2 & $76.28 \pm 4.3$ & $0.4165(2)$ & $4.458(1) \times 10^{38}$ \\
\hline 449 & 58729.034311978 & $563.3(2)$ & $1.92(2)$ & 0.05 & $97.1 \pm 7.5$ & 0.1864 ( 1 ) & $1.996(1) \times 10^{38}$ \\
\hline 450 & 58729.037216964 & $566.1(4)$ & $1.8(4)$ & 0.3 & $88.94 \pm 6.5$ & $0.1601(14)$ & $1.714(14) \times 10^{38}$ \\
\hline 452 & 58729.037340178 & $568.1(5)$ & $7.48(9)$ & 0.1 & $3.334 \pm 0.0$ & $0.0249(0)$ & $2.668(3) \times 10^{37}$ \\
\hline 453 & 58729.042565897 & $566.0(5)$ & $3.09(5)$ & 0.055 & $7.268 \pm 0.1$ & $0.0225(0)$ & $2.405(2) \times 10^{37}$ \\
\hline 454 & 58729.043570390 & $562.5(2)$ & $5.51(5)$ & 0.4 & $180.85 \pm 13.5$ & $0.9965(18)$ & $1.067(1) \times 10^{39}$ \\
\hline 455 & 58729.043570454 & $562.5(2)$ & $10.49(9)$ & 0.4 & $97.97 \pm 12.7$ & $1.0277(23)$ & $1.100(2) \times 10^{39}$ \\
\hline 456 & 58729.045838277 & $566.6(6)$ & $6.79(6)$ & 0.15 & $58.74 \pm 4.4$ & $0.3988(3)$ & $4.270(2) \times 10^{38}$ \\
\hline 457 & 58729.047381468 & $563.5(4)$ & $2.21(2)$ & 0.2 & $118.53 \pm 7.9$ & $0.2620(1)$ & $2.804(1) \times 10^{38}$ \\
\hline 458 & 58729.049558315 & $566.7(4)$ & $4.48(3)$ & 0.15 & $112.0 \pm 5.5$ & $0.5018(1)$ & $5.371(1) \times 10^{38}$ \\
\hline 459 & 58729.051243539 & $563.8(2)$ & $3.44(2)$ & 0.3 & $152.3 \pm 6.5$ & $0.5239(1)$ & $5.608(1) \times 10^{38}$ \\
\hline 460 & 58729.052212093 & $564.0(2)$ & $3.74(3)$ & 0.3 & $158.39 \pm 6.5$ & $0.5924(2)$ & $6.341(1) \times 10^{38}$ \\
\hline 461 & 58729.052212138 & $564.1(2)$ & $2.27(1)$ & 0.3 & $411.49 \pm 8.2$ & $0.9341(1)$ & $9.999(0) \times 10^{38}$ \\
\hline 462 & 58729.055188277 & $568.4(7)$ & $5.63(8)$ & 0.2 & $42.26 \pm 5.1$ & $0.2379(4)$ & $2.547(4) \times 10^{38}$ \\
\hline 463 & 58729.056098235 & $561.5(5)$ & $5.62(8)$ & 0.19 & $6.275 \pm 0.1$ & $0.0352(0)$ & $3.772(5) \times 10^{37}$ \\
\hline 464 & 58729.063070333 & $564.6(2)$ & $3.18(0)$ & 0.4 & $1015.45 \pm 7.1$ & $3.2291(0)$ & $3.457(0) \times 10^{39}$ \\
\hline 465 & 58729.063625838 & $569.4(8)$ & $8.41(6)$ & 0.15 & $67.67 \pm 4.4$ & $0.5691(3)$ & $6.092(3) \times 10^{38}$ \\
\hline 466 & 58729.065374501 & $569.8(5)$ & $7.13(9)$ & 0.23 & $13.597 \pm 0.2$ & $0.0970(1)$ & $1.038(1) \times 10^{38}$ \\
\hline 467 & 58729.065374568 & $568.7(6)$ & $6.17(9)$ & 0.2 & $33.54 \pm 5.0$ & $0.2069(5)$ & $2.215(5) \times 10^{38}$ \\
\hline 470 & 58729.072278756 & $563.4(2)$ & $2.88(2)$ & 0.25 & $116.19 \pm 7.7$ & $0.3346(2)$ & $3.582(1) \times 10^{38}$ \\
\hline 471 & 58729.073079694 & $564.9(3)$ & $2.1(0)$ & 0.3 & $583.18 \pm 9.3$ & $1.2247(0)$ & $1.311(0) \times 10^{39}$ \\
\hline 472 & 58729.076089351 & $566.4(5)$ & $3.11(3)$ & 0.3 & $83.6 \pm 7.6$ & $0.2600(3)$ & $2.783(2) \times 10^{38}$ \\
\hline 473 & 58729.078359904 & $566.0(5)$ & $3.08(4)$ & 0.15 & $12.738 \pm 0.1$ & $0.0392(1)$ & $4.198(5) \times 10^{37}$ \\
\hline 474 & 58729.080879470 & $568.5(5)$ & $4.31(6)$ & 0.19 & $12.439 \pm 0.1$ & $0.0536(1)$ & $5.740(7) \times 10^{37}$ \\
\hline 475 & 58730.871337073 & $568.1(5)$ & $7.98(9)$ & 0.1 & $9.091 \pm 0.1$ & $0.0725(1)$ & $7.764(13) \times 10^{37}$ \\
\hline 476 & 58730.873486930 & $567.0(5)$ & $6.88(8)$ & 0.15 & $57.11 \pm 5.7$ & $0.3929(5)$ & $4.206(4) \times 10^{38}$ \\
\hline 477 & 58730.878462279 & $567.6(5)$ & $3.19(5)$ & 0.09 & $10.18 \pm 0.1$ & $0.0325(0)$ & $3.476(5) \times 10^{37}$ \\
\hline 478 & 58730.880265341 & $569.5(8)$ & $8.43(9)$ & 0.05 & $38.7 \pm 4.9$ & $0.3262(6)$ & $3.492(6) \times 10^{38}$ \\
\hline 479 & 58730.880983008 & $568.1(5)$ & $1.79(3)$ & 0.2 & $7.233 \pm 0.1$ & $0.0129(0)$ & $1.383(1) \times 10^{37}$ \\
\hline 480 & 58730.881875450 & $566.0(5)$ & $3.84(6)$ & 0.105 & $5.511 \pm 0.1$ & $0.0212(0)$ & $2.265(3) \times 10^{37}$ \\
\hline 481 & 58730.885466103 & $565.9(5)$ & $6.75(9)$ & 0.11 & $7.485 \pm 0.1$ & $0.0505(1)$ & $5.405(7) \times 10^{37}$ \\
\hline 482 & 58730.886768291 & $563.4(5)$ & $1.3(1)$ & 0.4 & $280.7 \pm 17.5$ & $0.3649(2)$ & $3.906(1) \times 10^{38}$ \\
\hline 483 & 58730.886768315 & $563.9(4)$ & $4.49(1)$ & 0.4 & $602.34 \pm 7.7$ & $2.7045(1)$ & $2.895(0) \times 10^{39}$ \\
\hline 484 & 58730.886768625 & $566.0(4)$ & $3.81(1)$ & 0.4 & $306.64 \pm 6.9$ & $1.1683(1)$ & $1.251(0) \times 10^{39}$ \\
\hline 485 & 58730.890202679 & $569.8(5)$ & $7.0(9)$ & 0.24 & $8.688 \pm 0.1$ & $0.0608(1)$ & $6.510(8) \times 10^{37}$ \\
\hline 486 & 58730.890244815 & $566.1(7)$ & $4.49(4)$ & 0.2 & $80.56 \pm 6.3$ & 0.3617 ( 3 ) & $3.872(2) \times 10^{38}$ \\
\hline 487 & 58730.890299696 & $564.5(3)$ & $1.98(1)$ & 0.1 & $404.5 \pm 9.3$ & $0.8009(0)$ & $8.574(0) \times 10^{38}$ \\
\hline 488 & 58730.890619232 & $564.5(3)$ & $1.51(2)$ & 0.1 & $126.09 \pm 10.5$ & 0.1904 ( 2 ) & $2.038(1) \times 10^{38}$ \\
\hline 489 & 58730.890935087 & $568.9(8)$ & $10.11(9)$ & 0.2 & $49.34 \pm 4.3$ & $0.4988(5)$ & $5.340(4) \times 10^{38}$ \\
\hline 490 & 58730.891717875 & $565.7(4)$ & $5.09(5)$ & 0.1 & $74.08 \pm 5.8$ & $0.3771(3)$ & $4.036(2) \times 10^{38}$ \\
\hline 491 & 58730.891996590 & $570.0(10)$ & $12.28(9)$ & 0.22 & $63.84 \pm 3.8$ & $0.7840(3)$ & $8.392(3) \times 10^{38}$ \\
\hline 492 & 58730.892710817 & $565.3(11)$ & $12.41(9)$ & 0.1 & $32.13 \pm 3.7$ & $0.3987(6)$ & $4.268(6) \times 10^{38}$ \\
\hline 493 & 58730.893067453 & $568.6(6)$ & $7.02(9)$ & 0.2 & $37.48 \pm 5.3$ & $0.2631(6)$ & $2.817(6) \times 10^{38}$ \\
\hline 494 & 58730.894069353 & $565.0(5)$ & $7.51(9)$ & 0.195 & $6.339 \pm 0.1$ & $0.0476(1)$ & $5.096(5) \times 10^{37}$ \\
\hline 495 & 58730.894543674 & $566.9(3)$ & $3.56(3)$ & 0.25 & $100.85 \pm 6.8$ & $0.3590(2)$ & $3.843(2) \times 10^{38}$ \\
\hline 496 & 58730.894836599 & $566.3(6)$ & $7.81(9)$ & 0.25 & $44.67 \pm 4.6$ & $0.3489(4)$ & $3.735(4) \times 10^{38}$ \\
\hline 497 & 58730.894836760 & $566.3(6)$ & $3.56(3)$ & 0.3 & $93.33 \pm 6.7$ & $0.3322(2)$ & $3.557(2) \times 10^{38}$ \\
\hline 498 & 58730.899666023 & $567.7(6)$ & $9.28(8)$ & 0.4 & $60.76 \pm 4.1$ & $0.5638(3)$ & $6.036(3) \times 10^{38}$ \\
\hline
\end{tabular}


Table 1:

\begin{tabular}{|c|c|c|c|c|c|c|c|}
\hline Burst ID & $\begin{array}{l}\text { Burst time }^{a)} \\
\text { (MJD) }\end{array}$ & $\begin{array}{c}\mathrm{DM} \\
\left(\mathrm{pc} \mathrm{cm}^{-3}\right)\end{array}$ & $\begin{array}{l}\text { Width } \\
\text { (ms) }\end{array}$ & $\begin{array}{c}\text { Bandwidth }^{b)} \\
(\mathrm{GHz})\end{array}$ & $\begin{array}{c}\text { Peak Flux } \\
(\mathrm{mJy})\end{array}$ & $\begin{array}{c}\begin{array}{c}\text { Fluence } \\
(\mathrm{Jy} \mathrm{ms})\end{array} \\
\end{array}$ & $\begin{array}{c}\text { Energy } \\
\text { (erg) }\end{array}$ \\
\hline 499 & 58730.900487247 & $566.2(3)$ & $4.05(3)$ & 0.25 & $105.84 \pm 6.3$ & $0.4286(2)$ & $4.589(1) \times 10^{38}$ \\
\hline 500 & 58730.902144470 & $567.0(5)$ & $4.89(4)$ & 0.4 & $79.47 \pm 5.4$ & $0.3886(2)$ & $4.160(2) \times 10^{38}$ \\
\hline 501 & 58730.903022744 & $567.7(6)$ & 3.89 ( 3 ) & 0.15 & $112.65 \pm 6.2$ & $0.4382(2)$ & $4.691(1) \times 10^{38}$ \\
\hline 502 & 58730.903393843 & $566.1(4)$ & $3.2(2)$ & 0.15 & $130.08 \pm 7.0$ & $0.4163(1)$ & $4.456(1) \times 10^{38}$ \\
\hline 503 & 58730.905022970 & $568.1(5)$ & $4.2(6)$ & 0.07 & $10.029 \pm 0.1$ & $0.0421(1)$ & $4.505(6) \times 10^{37}$ \\
\hline 504 & 58730.905046852 & $566.0(5)$ & $2.98(4)$ & 0.105 & $10.013 \pm 0.1$ & $0.0298(0)$ & $3.193(4) \times 10^{37}$ \\
\hline 505 & 58730.906044512 & $562.4(5)$ & $3.08(4)$ & 0.13 & $12.501 \pm 0.1$ & $0.0385(1)$ & $4.117(5) \times 10^{37}$ \\
\hline 506 & 58730.906965636 & $564.4(2)$ & $3.51(0)$ & 0.3 & $1654.83 \pm 7.7$ & $5.8084(0)$ & $6.218(0) \times 10^{39}$ \\
\hline 507 & 58730.907008500 & $563.6(3)$ & $1.93(0)$ & 0.4 & $539.55 \pm 9.3$ & $1.0413(0)$ & $1.115(0) \times 10^{39}$ \\
\hline 508 & 58730.907545592 & $563.6(5)$ & $2.71(1)$ & 0.2 & $446.93 \pm 8.4$ & $1.2112(1)$ & $1.297(0) \times 10^{39}$ \\
\hline 509 & 58730.907844147 & $566.9(4)$ & $7.29(9)$ & 0.2 & $45.06 \pm 4.5$ & 0.3285 ( 4$)$ & $3.516(4) \times 10^{38}$ \\
\hline 510 & 58730.908489266 & $565.3(3)$ & 3.24 ( 1 ) & 0.3 & $221.41 \pm 7.0$ & $0.7174(1)$ & $7.679(0) \times 10^{38}$ \\
\hline 511 & 58732.868655452 & $561.9(5)$ & $5.35(8)$ & 0.18 & $6.087 \pm 0.1$ & $0.0325(1)$ & $3.483(5) \times 10^{37}$ \\
\hline 512 & 58732.868758525 & $562.8(5)$ & $5.94(9)$ & 0.09 & $6.961 \pm 0.1$ & $0.0414(1)$ & $4.430(7) \times 10^{37}$ \\
\hline 513 & 58732.868916895 & $566.0(5)$ & $3.76(5)$ & 0.17 & $10.114 \pm 0.1$ & $0.0381(1)$ & $4.073(6) \times 10^{37}$ \\
\hline 514 & 58732.869847142 & $566.0(5)$ & $4.69(7)$ & 0.21 & $17.479 \pm 0.2$ & $0.0820(1)$ & $8.778(13) \times 10^{37}$ \\
\hline 515 & 58732.870169445 & $566.0(5)$ & $6.74(9)$ & 0.26 & $25.356 \pm 0.3$ & $0.1708(3)$ & $1.829(2) \times 10^{38}$ \\
\hline 516 & 58732.870480021 & $566.0(5)$ & $3.54(5)$ & 0.17 & $17.748 \pm 0.2$ & $0.0627(1)$ & $6.717(10) \times 10^{37}$ \\
\hline 517 & 58732.870850606 & $566.0(5)$ & $2.1(3)$ & 0.1 & $17.771 \pm 0.2$ & $0.0373(1)$ & 3.995 ( 6$) \times 10^{37}$ \\
\hline 518 & 58732.871316874 & $566.3(5)$ & $3.49(5)$ & 0.105 & $14.689 \pm 0.2$ & $0.0513(1)$ & $5.489(8) \times 10^{37}$ \\
\hline 519 & 58732.876086431 & $566.0(5)$ & $3.13(5)$ & 0.105 & $18.6 \pm 0.2$ & $0.0582(1)$ & $6.228(8) \times 10^{37}$ \\
\hline 520 & 58732.876708190 & $565.4(5)$ & $5.54(8)$ & 0.35 & $11.127 \pm 0.1$ & $0.0616(1)$ & $6.594(9) \times 10^{37}$ \\
\hline 521 & 58732.877561933 & $566.0(5)$ & $2.97(4)$ & 0.07 & $12.889 \pm 0.2$ & $0.0383(1)$ & $4.095(5) \times 10^{37}$ \\
\hline 522 & 58732.878372149 & $563.7(5)$ & $7.2(9)$ & 0.08 & $6.083 \pm 0.1$ & $0.0438(1)$ & $4.689(6) \times 10^{37}$ \\
\hline 523 & 58732.878786935 & $569.4(5)$ & $8.56(9)$ & 0.14 & $8.975 \pm 0.1$ & $0.0768(1)$ & $8.224(11) \times 10^{37}$ \\
\hline 524 & 58732.878953286 & $566.0(5)$ & $3.63(5)$ & 0.16 & $27.168 \pm 0.3$ & $0.0985(1)$ & $1.055(1) \times 10^{38}$ \\
\hline 525 & 58732.879987451 & $566.0(5)$ & $4.48(7)$ & 0.405 & $24.246 \pm 0.3$ & $0.1086(1)$ & $1.162(1) \times 10^{38}$ \\
\hline 526 & 58732.880153199 & $566.0(5)$ & $4.69(7)$ & 0.2 & $24.041 \pm 0.3$ & $0.1127(2)$ & $1.206(1) \times 10^{38}$ \\
\hline 527 & 58732.952323411 & $565.9(5)$ & $7.53(9)$ & 0.13 & $7.779 \pm 0.1$ & $0.0586(1)$ & $6.271(5) \times 10^{37}$ \\
\hline 528 & 58732.953026073 & $565.7(4)$ & $3.18(3)$ & 0.1 & $64.35 \pm 5.6$ & $0.2046(2)$ & $2.191(1) \times 10^{38}$ \\
\hline 529 & 58732.953170125 & $566.0(5)$ & $4.87(7)$ & 0.2 & $16.394 \pm 0.1$ & $0.0798(1)$ & $8.547(7) \times 10^{37}$ \\
\hline 530 & 58732.953423290 & $565.2(3)$ & $1.65(3)$ & 0.15 & $50.09 \pm 7.6$ & $0.0827(2)$ & $8.847(23) \times 10^{37}$ \\
\hline 531 & 58732.954034308 & $561.5(5)$ & $4.3(6)$ & 0.165 & $20.255 \pm 0.2$ & $0.0872(1)$ & $9.331(8) \times 10^{37}$ \\
\hline 532 & 58732.954245471 & $566.7(5)$ & $2.96(4)$ & 0.11 & $6.917 \pm 0.1$ & $0.0205(0)$ & $2.192(1) \times 10^{37}$ \\
\hline 533 & 58732.954344729 & $564.1(5)$ & $5.71(8)$ & 0.2 & $9.535 \pm 0.1$ & $0.0544(0)$ & $5.827(5) \times 10^{37}$ \\
\hline 534 & 58732.954595908 & $567.7(9)$ & $6.27(5)$ & 0.2 & $67.24 \pm 4.0$ & $0.4216(2)$ & $4.513(1) \times 10^{38}$ \\
\hline 535 & 58732.954869859 & $566.0(5)$ & $6.4(9)$ & 0.11 & $15.626 \pm 0.1$ & $0.0999(1)$ & $1.070(0) \times 10^{38}$ \\
\hline 536 & 58732.954927574 & $566.0(5)$ & $5.28(8)$ & 0.2 & $15.808 \pm 0.1$ & $0.0834(1)$ & $8.927(8) \times 10^{37}$ \\
\hline 537 & 58732.955664980 & $565.4(6)$ & $8.0(2)$ & 0.1 & $158.47 \pm 3.6$ & $1.2678(1)$ & $1.357(0) \times 10^{39}$ \\
\hline 538 & 58732.955665081 & $566.0(5)$ & $7.72(9)$ & 0.18 & $50.363 \pm 0.4$ & $0.3888(4)$ & $4.162(3) \times 10^{38}$ \\
\hline 539 & 58732.956126048 & $564.0(2)$ & $2.66(1)$ & 0.15 & $142.2 \pm 5.8$ & $0.3782(1)$ & $4.049(0) \times 10^{38}$ \\
\hline 540 & 58732.956126058 & $566.0(5)$ & $3.77(5)$ & 0.21 & $29.656 \pm 0.2$ & $0.1119(1)$ & $1.198(1) \times 10^{38}$ \\
\hline 541 & 58732.956223954 & $566.0(5)$ & $2.36(3)$ & 0.13 & $14.752 \pm 0.1$ & $0.0348(0)$ & $3.724(3) \times 10^{37}$ \\
\hline 542 & 58732.956414380 & $564.2(3)$ & $3.51(4)$ & 0.22 & $65.14 \pm 5.5$ & $0.2286(2)$ & $2.448(2) \times 10^{38}$ \\
\hline 543 & 58732.956414419 & $561.5(5)$ & $12.51(9)$ & 0.19 & $12.831 \pm 0.1$ & $0.1606(1)$ & $1.719(1) \times 10^{38}$ \\
\hline 544 & 58732.957417040 & $566.0(5)$ & $2.34(3)$ & 0.16 & $11.181 \pm 0.1$ & $0.0262(0)$ & $2.806(2) \times 10^{37}$ \\
\hline 545 & 58732.958590896 & $566.0(5)$ & $3.15(5)$ & 0.3 & $17.769 \pm 0.1$ & $0.0559(0)$ & $5.984(5) \times 10^{37}$ \\
\hline 546 & 58732.959134328 & $567.5(10)$ & $11.33(8)$ & 0.25 & $56.23 \pm 3.2$ & 0.6371 ( 3 ) & $6.820(2) \times 10^{38}$ \\
\hline 547 & 58732.959351161 & $567.2(5)$ & $7.3(9)$ & 0.16 & $7.21 \pm 0.1$ & $0.0526(0)$ & $5.631(5) \times 10^{37}$ \\
\hline 548 & 58732.959623565 & $569.8(5)$ & $3.67(5)$ & 0.05 & $5.192 \pm 0.0$ & $0.0190(0)$ & $2.037(1) \times 10^{37}$ \\
\hline 549 & 58732.959754819 & $566.0(5)$ & $3.03(4)$ & 0.03 & $20.321 \pm 0.2$ & $0.0616(1)$ & $6.592(5) \times 10^{37}$ \\
\hline 550 & 58732.960040324 & $568.4(5)$ & $4.81(3)$ & 0.1 & $81.57 \pm 4.5$ & $0.3923(1)$ & $4.200(1) \times 10^{38}$ \\
\hline 551 & 58732.960192916 & $564.1(5)$ & $6.47(9)$ & 0.14 & $11.596 \pm 0.1$ & $0.0750(1)$ & $8.031(7) \times 10^{37}$ \\
\hline 552 & 58732.960739959 & $565.4(5)$ & $3.53(5)$ & 0.12 & $10.955 \pm 0.1$ & $0.0387(0)$ & $4.145(3) \times 10^{37}$ \\
\hline 553 & 58732.962689960 & $565.4(5)$ & $4.14(6)$ & 0.21 & $394.235 \pm 3.0$ & $1.6337(14)$ & $1.749(1) \times 10^{39}$ \\
\hline 554 & 58732.962867196 & $566.0(5)$ & $2.83(4)$ & 0.13 & $15.453 \pm 0.1$ & $0.0437(0)$ & $4.680(4) \times 10^{37}$ \\
\hline 555 & 58732.962914876 & $563.7(5)$ & $2.3(3)$ & 0.15 & $9.943 \pm 0.1$ & $0.0229(0)$ & $2.453(2) \times 10^{37}$ \\
\hline 556 & 58732.962966373 & $566.2(7)$ & $4.18(5)$ & 0.35 & $50.14 \pm 5.2$ & $0.2096(3)$ & $2.244(2) \times 10^{38}$ \\
\hline 557 & 58732.963464708 & $565.9(5)$ & $6.32(9)$ & 0.29 & $12.091 \pm 0.1$ & $0.0764(1)$ & $8.176(7) \times 10^{37}$ \\
\hline 558 & 58732.963779691 & $568.5(5)$ & $3.95(6)$ & 0.17 & $15.509 \pm 0.1$ & $0.0612(1)$ & $6.553(5) \times 10^{37}$ \\
\hline 559 & 58732.963782645 & $567.2(5)$ & $8.3(9)$ & 0.17 & $10.432 \pm 0.1$ & $0.0866(1)$ & $9.266(7) \times 10^{37}$ \\
\hline 560 & 58732.964678668 & $567.6(5)$ & $4.18(6)$ & 0.1 & $9.14 \pm 0.1$ & $0.0382(0)$ & $4.089(3) \times 10^{37}$ \\
\hline 561 & 58732.964964135 & $564.5(5)$ & $3.72(5)$ & 0.12 & $17.344 \pm 0.1$ & $0.0645(1)$ & $6.909(5) \times 10^{37}$ \\
\hline
\end{tabular}


Table 1:

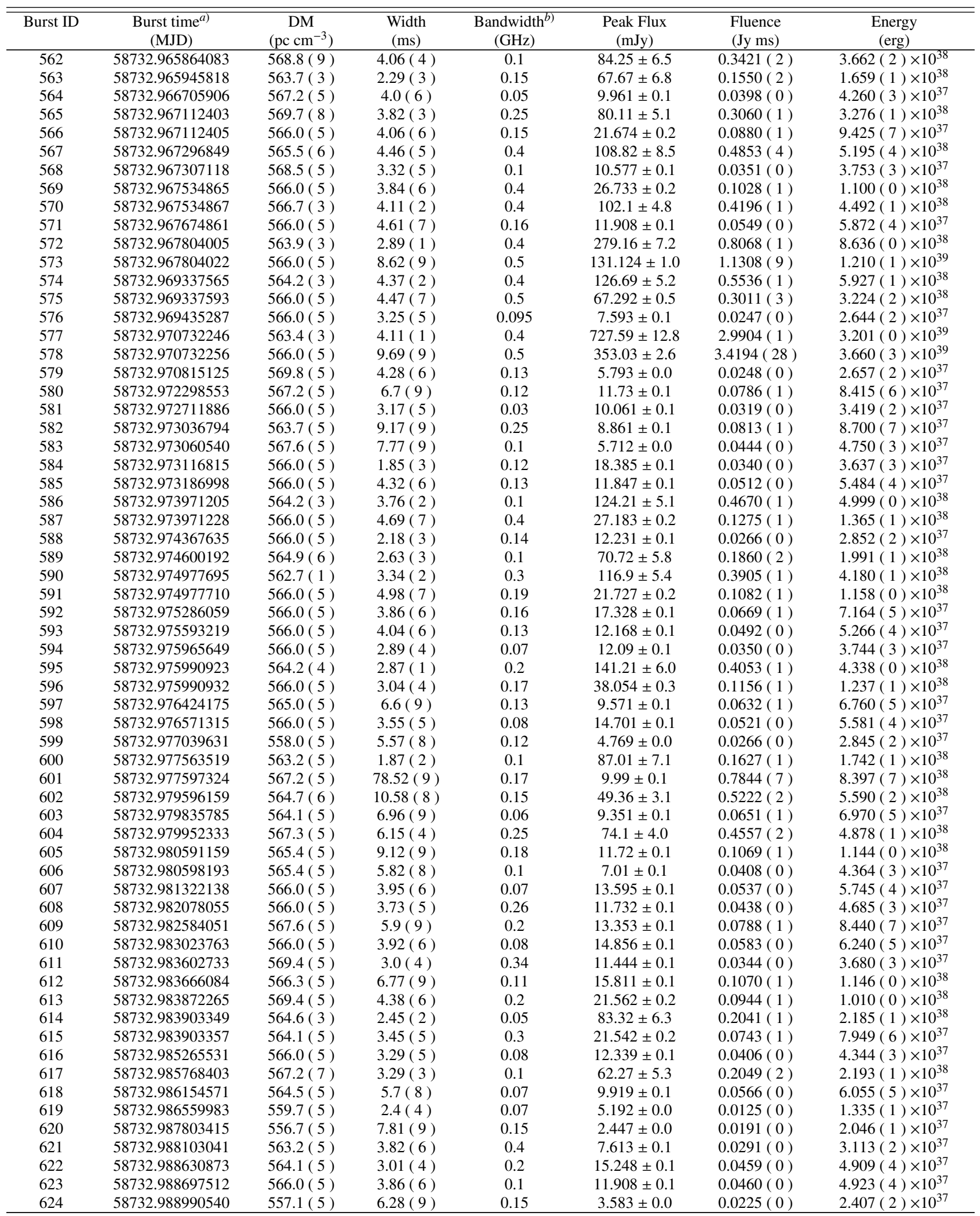


Table 1:

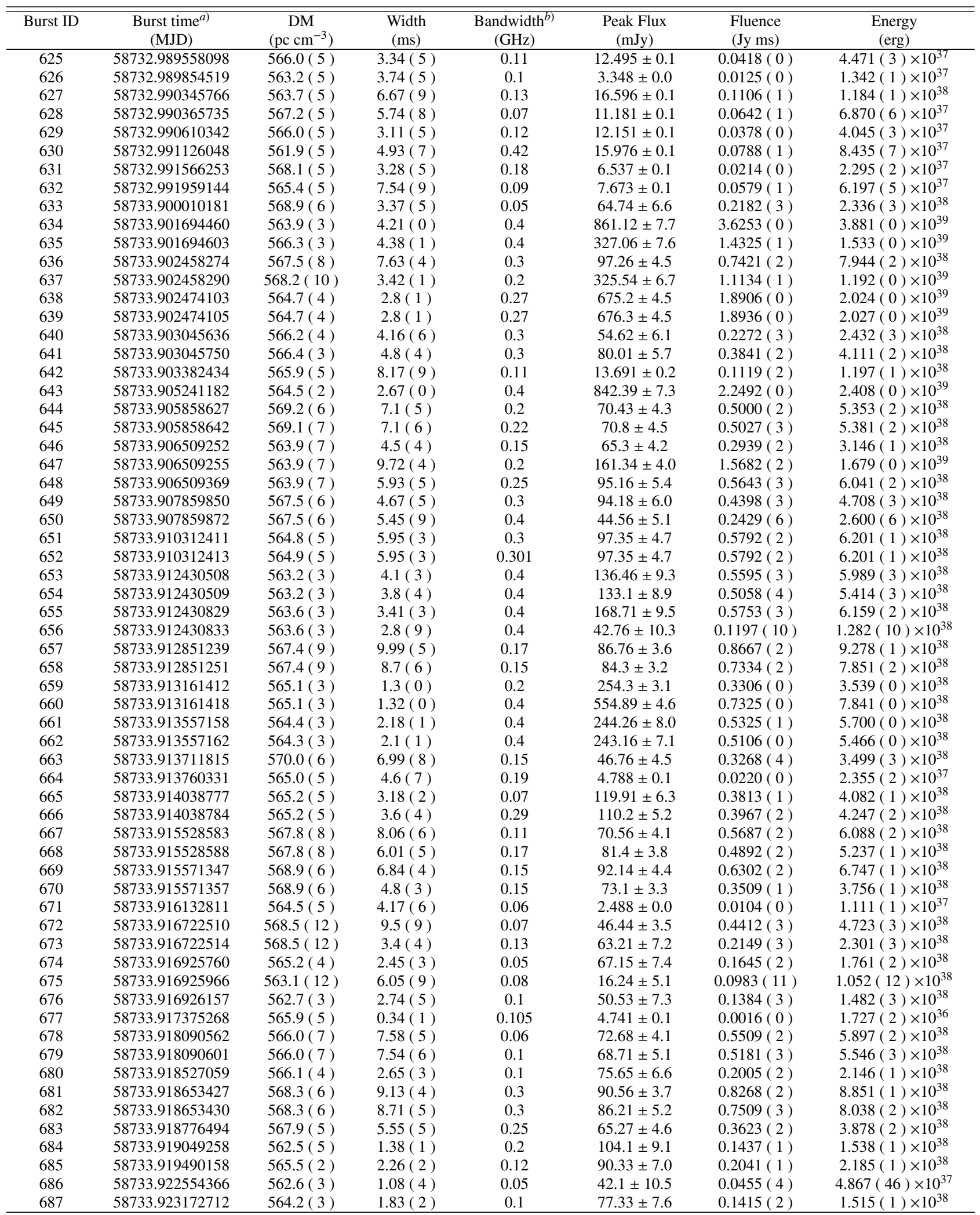


Table 1:

\begin{tabular}{|c|c|c|c|c|c|c|c|}
\hline Burst ID & $\begin{array}{c}\text { Burst time }^{a)} \\
\text { (MJD) }\end{array}$ & $\begin{array}{c}\mathrm{DM} \\
(\mathrm{pc} \mathrm{cm}-3)\end{array}$ & $\begin{array}{l}\text { Width } \\
\text { (ms) }\end{array}$ & $\begin{array}{c}\left.\text { Bandwidth }^{b}\right) \\
(\mathrm{GHz})\end{array}$ & $\begin{array}{c}\begin{array}{c}\text { Peak Flux } \\
(\mathrm{mJy})\end{array} \\
\end{array}$ & $\begin{array}{c}\text { Fluence } \\
\text { (Jy ms) }\end{array}$ & $\begin{array}{c}\text { Energy } \\
\text { (erg) }\end{array}$ \\
\hline 688 & 58733.924198436 & $565.4(4)$ & $3.26(7)$ & 0.2 & $32.48 \pm 6.0$ & $0.1059(4)$ & $1.133(4) \times 10^{38}$ \\
\hline 689 & 58733.924198899 & $566.4(11)$ & $2.33(5)$ & 0.15 & $45.16 \pm 7.9$ & $0.1052(4)$ & $1.126(4) \times 10^{38}$ \\
\hline 690 & 58733.924199240 & $566.4(11)$ & $4.41(6)$ & 0.15 & $50.19 \pm 5.5$ & $0.2213(3)$ & $2.369(3) \times 10^{38}$ \\
\hline 691 & 58733.924199397 & $566.4(11)$ & $4.24(5)$ & 0.15 & $54.08 \pm 5.6$ & $0.2293(3)$ & $2.455(3) \times 10^{38}$ \\
\hline 692 & 58733.924411719 & $565.4(4)$ & $2.67(1)$ & 0.15 & $336.42 \pm 6.5$ & $0.8982(0)$ & $9.616(0) \times 10^{38}$ \\
\hline 693 & 58733.924411721 & $565.4(4)$ & $1.01(2)$ & 0.15 & $59.61 \pm 5.1$ & $0.0602(1)$ & $6.445(11) \times 10^{37}$ \\
\hline 695 & 58733.926691786 & $562.4(5)$ & $4.4(6)$ & 0.05 & $11.228 \pm 0.1$ & $0.0494(1)$ & $5.292(5) \times 10^{37}$ \\
\hline 696 & 58733.927318696 & $564.2(2)$ & $2.01(2)$ & 0.1 & $73.8 \pm 7.2$ & $0.1483(2)$ & $1.588(1) \times 10^{38}$ \\
\hline 697 & 58733.927690364 & $555.8(5)$ & $6.18(9)$ & 0.3 & $4.081 \pm 0.0$ & $0.0252(0)$ & $2.698(3) \times 10^{37}$ \\
\hline 698 & 58733.928217035 & $566.4(5)$ & $10.16(9)$ & 0.3 & $36.58 \pm 3.4$ & $0.3716(4)$ & $3.978(4) \times 10^{38}$ \\
\hline 699 & 58733.928217205 & $564.5(4)$ & $3.06(4)$ & 0.3 & $56.07 \pm 6.0$ & $0.1716(2)$ & $1.837(2) \times 10^{38}$ \\
\hline 700 & 58733.928457350 & $569.4(7)$ & $6.77(5)$ & 0.2 & $67.5 \pm 3.9$ & $0.4570(2)$ & $4.892(1) \times 10^{38}$ \\
\hline 701 & 58733.929699545 & $566.0(5)$ & $3.45(5)$ & 0.18 & $8.216 \pm 0.1$ & $0.0283(0)$ & $3.033(3) \times 10^{37}$ \\
\hline 702 & 58733.930019841 & $563.8(5)$ & $1.84(2)$ & 0.05 & $85.19 \pm 7.4$ & $0.1568(1)$ & $1.678(1) \times 10^{38}$ \\
\hline 704 & 58733.930442763 & $562.3(5)$ & $3.26(5)$ & 0.15 & $5.94 \pm 0.1$ & $0.0194(0)$ & $2.072(2) \times 10^{37}$ \\
\hline 705 & 58733.930559496 & $564.8(6)$ & $5.89(4)$ & 0.1 & $77.85 \pm 4.2$ & $0.4585(2)$ & $4.909(1) \times 10^{38}$ \\
\hline 706 & 58733.930626087 & $566.0(5)$ & $7.87(9)$ & 0.1 & $22.839 \pm 0.2$ & $0.1797(2)$ & $1.923(2) \times 10^{38}$ \\
\hline 707 & 58733.930626091 & $564.6(2)$ & $2.39(2)$ & 0.15 & $111.52 \pm 6.4$ & $0.2665(1)$ & $2.853(1) \times 10^{38}$ \\
\hline 708 & 58733.930637383 & $566.4(12)$ & $7.83(9)$ & 0.15 & $36.09 \pm 3.7$ & $0.2826(4)$ & $3.025(3) \times 10^{38}$ \\
\hline 709 & 58733.930647496 & $566.0(5)$ & $2.92(4)$ & 0.095 & $13.409 \pm 0.1$ & $0.0392(0)$ & $4.194(4) \times 10^{37}$ \\
\hline 710 & 58733.930933352 & $568.1(5)$ & $7.21(9)$ & 0.29 & $15.148 \pm 0.1$ & $0.1092(1)$ & $1.168(1) \times 10^{38}$ \\
\hline 711 & 58733.930933372 & $568.3(7)$ & $4.76(5)$ & 0.05 & $52.15 \pm 4.7$ & $0.2482(2)$ & $2.657(2) \times 10^{38}$ \\
\hline 712 & 58733.931072280 & $566.0(5)$ & $3.94(6)$ & 0.18 & $8.408 \pm 0.1$ & $0.0331(0)$ & $3.548(3) \times 10^{37}$ \\
\hline 713 & 58733.931243100 & $565.6(5)$ & $4.61(7)$ & 0.07 & $18.113 \pm 0.2$ & $0.0836(1)$ & $8.948(9) \times 10^{37}$ \\
\hline 714 & 58735.011288763 & $569.4(5)$ & $8.71(9)$ & 0.11 & $10.801 \pm 0.1$ & $0.0941(1)$ & $1.007(1) \times 10^{38}$ \\
\hline 715 & 58735.014135350 & $564.0(7)$ & $7.6(9)$ & 0.2 & $41.57 \pm 4.1$ & $0.3159(4)$ & $3.382(3) \times 10^{38}$ \\
\hline 716 & 58735.015124262 & $564.2(3)$ & $2.56(1)$ & 0.4 & $220.16 \pm 6.6$ & $0.5636(1)$ & $6.033(0) \times 10^{38}$ \\
\hline 717 & 58735.016395653 & $567.7(9)$ & $4.34(8)$ & 0.4 & $35.3 \pm 5.8$ & $0.1532(5)$ & $1.640(5) \times 10^{38}$ \\
\hline 718 & 58735.016396008 & $567.3(4)$ & $12.77(9)$ & 0.32 & $31.99 \pm 3.2$ & $0.4085(5)$ & $4.373(5) \times 10^{38}$ \\
\hline 719 & 58735.017492352 & $568.6(8)$ & $6.24(5)$ & 0.2 & $60.09 \pm 4.2$ & $0.3750(2)$ & $4.014(2) \times 10^{38}$ \\
\hline 722 & 58735.018564200 & $565.6(6)$ & $7.09(5)$ & 0.25 & $63.98 \pm 3.9$ & $0.4536(2)$ & $4.856(2) \times 10^{38}$ \\
\hline 723 & 58735.019356893 & $564.4(4)$ & $5.92(4)$ & 0.2 & $72.51 \pm 4.5$ & $0.4293(2)$ & $4.595(2) \times 10^{38}$ \\
\hline 724 & 58735.019820485 & $568.1(5)$ & $3.42(5)$ & 0.18 & $7.845 \pm 0.1$ & $0.0268(0)$ & $2.874(3) \times 10^{37}$ \\
\hline 725 & 58735.021348982 & $570.0(11)$ & $8.03(7)$ & 0.2 & $51.18 \pm 3.9$ & $0.4110(3)$ & $4.399(2) \times 10^{38}$ \\
\hline 726 & 58735.021948411 & $564.5(6)$ & $3.55(3)$ & 0.15 & $90.8 \pm 5.6$ & $0.3223(1)$ & $3.451(1) \times 10^{38}$ \\
\hline 727 & 58735.022426651 & $568.3(5)$ & $7.86(4)$ & 0.3 & $90.07 \pm 4.0$ & $0.7079(2)$ & $7.578(1) \times 10^{38}$ \\
\hline 728 & 58735.022743110 & $564.6(5)$ & $2.77(3)$ & 0.05 & $66.04 \pm 6.4$ & $0.1829(2)$ & $1.958(2) \times 10^{38}$ \\
\hline 729 & 58735.023312496 & $565.1(4)$ & $2.72(3)$ & 0.1 & $71.49 \pm 6.6$ & $0.1945(2)$ & $2.082(2) \times 10^{38}$ \\
\hline 730 & 58735.023335389 & $566.7(4)$ & $3.35(2)$ & 0.2 & $94.69 \pm 5.7$ & $0.3172(1)$ & $3.396(1) \times 10^{38}$ \\
\hline 731 & 58735.025054035 & $568.4(6)$ & $3.66(5)$ & 0.23 & $54.53 \pm 5.9$ & $0.1996(3)$ & $2.136(2) \times 10^{38}$ \\
\hline 732 & 58735.026639952 & $566.7(7)$ & $21.27(9)$ & 0.4 & $31.1 \pm 2.6$ & $0.6615(6)$ & $7.081(6) \times 10^{38}$ \\
\hline 733 & 58735.027081056 & $563.9(3)$ & $2.58(0)$ & 0.35 & $679.99 \pm 7.4$ & $1.7544(0)$ & $1.878(0) \times 10^{39}$ \\
\hline 734 & 58735.028468657 & $566.1(7)$ & $5.38(3)$ & 0.18 & $89.44 \pm 4.7$ & $0.4812(2)$ & $5.151(1) \times 10^{38}$ \\
\hline 735 & 58735.028658842 & $569.8(5)$ & $3.64(5)$ & 0.09 & $4.935 \pm 0.1$ & $0.0179(0)$ & $1.921(2) \times 10^{37}$ \\
\hline 736 & 58735.029045242 & $562.8(5)$ & $6.74(9)$ & 0.13 & $7.451 \pm 0.1$ & $0.0502(1)$ & $5.373(6) \times 10^{37}$ \\
\hline 737 & 58735.031125232 & $562.3(2)$ & $0.94(1)$ & 0.35 & $132.12 \pm 13.8$ & $0.1242(2)$ & $1.329(1) \times 10^{38}$ \\
\hline 738 & 58735.031125298 & $564.3(5)$ & $3.16(1)$ & 0.3 & $325.62 \pm 7.5$ & $1.0290(1)$ & $1.101(0) \times 10^{39}$ \\
\hline 739 & 58735.031889481 & $562.6(4)$ & $1.99(2)$ & 0.1 & $95.54 \pm 8.0$ & $0.1901(2)$ & $2.035(1) \times 10^{38}$ \\
\hline 740 & 58735.032261719 & $567.6(4)$ & $4.07(3)$ & 0.15 & $97.26 \pm 5.6$ & $0.3958(2)$ & $4.237(1) \times 10^{38}$ \\
\hline 741 & 58735.032660540 & $566.4(7)$ & $9.3(7)$ & 0.15 & $60.72 \pm 3.7$ & $0.5647(3)$ & $6.045(2) \times 10^{38}$ \\
\hline 742 & 58735.032661221 & $568.3(12)$ & $5.12(9)$ & 0.1 & $34.67 \pm 5.2$ & $0.1775(5)$ & $1.900(4) \times 10^{38}$ \\
\hline 743 & 58735.036492035 & $566.2(4)$ & $3.39(3)$ & 0.2 & $76.24 \pm 6.6$ & $0.2585(2)$ & $2.767(2) \times 10^{38}$ \\
\hline 744 & 58735.036766864 & $567.4(9)$ & $6.28(2)$ & 0.4 & $155.11 \pm 5.1$ & $0.9741(1)$ & $1.043(0) \times 10^{39}$ \\
\hline 745 & 58735.037242917 & $567.4(7)$ & $5.02(4)$ & 0.2 & $77.55 \pm 5.6$ & $0.3893(2)$ & $4.167(2) \times 10^{38}$ \\
\hline 746 & 58735.039089158 & $567.3(6)$ & $4.76(5)$ & 0.05 & $66.89 \pm 5.7$ & $0.3184(3)$ & $3.408(2) \times 10^{38}$ \\
\hline 747 & 58735.040177746 & $566.5(3)$ & $4.49(4)$ & 0.3 & $69.99 \pm 5.7$ & $0.3143(2)$ & $3.364(2) \times 10^{38}$ \\
\hline 748 & 58735.040441411 & $568.3(3)$ & $11.01(9)$ & 0.3 & $44.35 \pm 3.7$ & $0.4883(4)$ & $5.227(4) \times 10^{38}$ \\
\hline 749 & 58735.043385137 & $568.2(8)$ & $25.8(9)$ & 0.25 & $36.28 \pm 2.8$ & $0.9360(8)$ & $1.002(0) \times 10^{39}$ \\
\hline 750 & 58735.044176394 & $563.0(5)$ & $3.71(3)$ & 0.25 & $90.52 \pm 6.5$ & $0.3358(2)$ & $3.595(2) \times 10^{38}$ \\
\hline
\end{tabular}


Table 1:

\begin{tabular}{|c|c|c|c|c|c|c|c|}
\hline Burst ID & $\begin{array}{l}\text { Burst time }^{a)} \\
\text { (MJD) }\end{array}$ & $\begin{array}{c}\mathrm{DM} \\
\left(\mathrm{pc} \mathrm{cm}^{-3}\right)\end{array}$ & $\begin{array}{l}\text { Width } \\
\text { (ms) }\end{array}$ & $\begin{array}{c}\text { Bandwidth }^{b)} \\
(\mathrm{GHz})\end{array}$ & $\begin{array}{c}\text { Peak Flux } \\
(\mathrm{mJy})\end{array}$ & $\begin{array}{c}\begin{array}{c}\text { Fluence } \\
(\mathrm{Jy} \mathrm{ms})\end{array} \\
\end{array}$ & $\begin{array}{c}\text { Energy } \\
\text { (erg) }\end{array}$ \\
\hline 751 & 58735.046093009 & $565.0(5)$ & $7.5(9)$ & 0.132 & $6.538 \pm 0.1$ & $0.0491(1)$ & $5.252(7) \times 10^{37}$ \\
\hline 752 & 58735.047246612 & $565.9(7)$ & $9.53(5)$ & 0.15 & $90.65 \pm 4.0$ & $0.8639(2)$ & $9.248(2) \times 10^{38}$ \\
\hline 753 & 58736.967291360 & $564.8(5)$ & 3.41 ( 1 ) & 0.3 & $501.56 \pm 6.6$ & $1.7103(0)$ & $1.831(0) \times 10^{39}$ \\
\hline 754 & 58736.967865417 & $569.0(5)$ & $5.03(7)$ & 0.15 & $3.281 \pm 0.0$ & $0.0165(0)$ & $1.768(1) \times 10^{37}$ \\
\hline 755 & 58736.969519157 & $569.5(10)$ & $8.85(8)$ & 0.11 & $44.39 \pm 3.2$ & $0.3928(3)$ & $4.205(2) \times 10^{38}$ \\
\hline 756 & 58736.971724401 & $564.7(5)$ & $2.91(3)$ & 0.4 & $57.48 \pm 5.6$ & $0.1673(2)$ & $1.791(1) \times 10^{38}$ \\
\hline 757 & 58736.972247061 & $567.2(5)$ & $5.22(8)$ & 0.26 & $4.12 \pm 0.0$ & $0.0215(0)$ & $2.304(1) \times 10^{37}$ \\
\hline 758 & 58736.973547441 & $563.2(5)$ & $2.72(3)$ & 0.05 & $75.85 \pm 6.5$ & $0.2063(2)$ & $2.209(2) \times 10^{38}$ \\
\hline 759 & 58736.975329770 & $566.0(5)$ & $3.39(5)$ & 0.13 & $6.434 \pm 0.1$ & $0.0218(0)$ & $2.335(1) \times 10^{37}$ \\
\hline 760 & 58736.975374498 & $566.5(5)$ & $4.58(6)$ & 0.45 & $46.68 \pm 4.8$ & $0.2138(3)$ & $2.289(2) \times 10^{38}$ \\
\hline 761 & 58736.979508692 & $564.0(4)$ & 2.89 (1) & 0.45 & $256.92 \pm 6.6$ & $0.7425(1)$ & $7.948(0) \times 10^{38}$ \\
\hline 762 & 58736.979508757 & $564.3(5)$ & $5.82(3)$ & 0.35 & $151.77 \pm 4.6$ & $0.8833(1)$ & $9.456(1) \times 10^{38}$ \\
\hline 763 & 58736.980956491 & $564.3(3)$ & $2.86(1)$ & 0.3 & $324.33 \pm 6.2$ & $0.9276(0)$ & $9.930(0) \times 10^{38}$ \\
\hline 764 & 58736.981901942 & $567.2(6)$ & $7.33(7)$ & 0.4 & $47.62 \pm 3.7$ & $0.3491(3)$ & $3.737(2) \times 10^{38}$ \\
\hline 765 & 58736.983522414 & $565.4(5)$ & 9.67 ( 8 ) & 0.25 & $47.4 \pm 3.3$ & $0.4584(3)$ & $4.907(2) \times 10^{38}$ \\
\hline 766 & 58736.983880206 & 566.7 ( 5$)$ & 4.19 ( 4$)$ & 0.12 & $58.79 \pm 4.8$ & $0.2463(2)$ & $2.637(2) \times 10^{38}$ \\
\hline 767 & 58736.984330166 & $566.7(4)$ & 7.06 ( 6$)$ & 0.15 & $50.33 \pm 3.8$ & $0.3553(2)$ & $3.804(2) \times 10^{38}$ \\
\hline 768 & 58736.985641172 & $567.7(5)$ & $5.03(7)$ & 0.25 & $40.24 \pm 4.4$ & $0.2024(3)$ & $2.167(3) \times 10^{38}$ \\
\hline 769 & 58736.988208149 & $562.2(2)$ & $4.46(5)$ & 0.11 & $53.78 \pm 4.9$ & 0.2399 ( 2 ) & $2.568(2) \times 10^{38}$ \\
\hline 770 & 58736.988393799 & $569.8(7)$ & $6.87(8)$ & 0.1 & $40.01 \pm 3.8$ & $0.2749(3)$ & $2.942(3) \times 10^{38}$ \\
\hline 771 & 58736.990630128 & 569.4 ( 5 ) & $5.92(9)$ & 0.06 & $6.828 \pm 0.1$ & $0.0405(0)$ & $4.330(4) \times 10^{37}$ \\
\hline 772 & 58736.992073771 & $563.6(2)$ & $3.52(2)$ & 0.35 & $137.12 \pm 5.4$ & $0.4827(1)$ & $5.167(0) \times 10^{38}$ \\
\hline 773 & 58736.992913357 & $568.0(5)$ & 3.65 ( 3 ) & 0.15 & $70.28 \pm 5.0$ & $0.2565(2)$ & $2.746(1) \times 10^{38}$ \\
\hline 774 & 58736.992944792 & $563.5(2)$ & $2.99(3)$ & 0.15 & $80.17 \pm 5.7$ & $0.2397(1)$ & $2.566(1) \times 10^{38}$ \\
\hline 775 & 58736.994828738 & $563.6(1)$ & $3.0(0)$ & 0.4 & $836.42 \pm 6.3$ & $2.5093(0)$ & $2.686(0) \times 10^{39}$ \\
\hline 776 & 58736.995193502 & $566.4(7)$ & $2.91(2)$ & 0.2 & $127.86 \pm 6.1$ & $0.3721(1)$ & $3.983(1) \times 10^{38}$ \\
\hline 777 & 58736.998264862 & 564.7 ( 4) & $3.97(0)$ & 0.4 & $945.19 \pm 7.4$ & $3.7524(0)$ & $4.017(0) \times 10^{39}$ \\
\hline 778 & 58737.000755111 & $565.9(5)$ & $4.23(4)$ & 0.45 & $67.07 \pm 4.8$ & $0.2837(2)$ & $3.037(1) \times 10^{38}$ \\
\hline 779 & 58737.000755868 & 567.5 ( 6 ) & $3.12(5)$ & 0.25 & $41.75 \pm 5.7$ & 0.1303 ( 3 ) & $1.394(3) \times 10^{38}$ \\
\hline 780 & 58737.001628415 & 567.7 ( 4 ) & 4.64 ( 3 ) & 0.2 & $87.06 \pm 4.4$ & $0.4040(1)$ & $4.324(1) \times 10^{38}$ \\
\hline 781 & 58737.003558913 & $565.9(4)$ & $6.35(6)$ & 0.2 & $51.04 \pm 4.3$ & $0.3241(3)$ & $3.469(2) \times 10^{38}$ \\
\hline 782 & 58738.959535574 & $566.0(5)$ & $4.49(7)$ & 0.2 & $10.151 \pm 0.1$ & $0.0456(0)$ & $4.879(4) \times 10^{37}$ \\
\hline 783 & 58738.959716725 & $564.5(5)$ & $7.61(9)$ & 0.17 & $8.113 \pm 0.1$ & $0.0618(1)$ & $6.613(5) \times 10^{37}$ \\
\hline 784 & 58738.960148438 & $559.7(5)$ & $6.61(9)$ & 0.17 & $10.402 \pm 0.1$ & $0.0688(1)$ & $7.365(6) \times 10^{37}$ \\
\hline 785 & 58738.960380572 & 567.9 ( 3$)$ & $8.59(9)$ & 0.15 & $7.52 \pm 0.6$ & $0.0646(1)$ & $6.915(5) \times 10^{37}$ \\
\hline 786 & 58738.960380647 & $566.9(4)$ & $6.31(8)$ & 0.21 & $47.31 \pm 2.8$ & $0.2985(2)$ & $3.196(2) \times 10^{38}$ \\
\hline 787 & 58738.960430203 & 568.7 ( 8 ) & $6.81(9)$ & 0.15 & $4.9 \pm 0.7$ & $0.0334(1)$ & $3.572(8) \times 10^{37}$ \\
\hline 788 & 58738.960432021 & 564.7 ( 3$)$ & $3.38(6)$ & 0.1 & $6.16 \pm 0.9$ & $0.0208(1)$ & $2.229(5) \times 10^{37}$ \\
\hline 789 & 58738.961418421 & $565.6(8)$ & $8.15(9)$ & 0.15 & $5.81 \pm 0.7$ & $0.0474(1)$ & $5.069(8) \times 10^{37}$ \\
\hline 790 & 58738.963365844 & $565.8(12)$ & 3.95 ( 6 ) & 0.05 & $6.81 \pm 0.8$ & $0.0269(0)$ & $2.880(4) \times 10^{37}$ \\
\hline 791 & 58738.963680861 & $565.8(4)$ & $5.77(7)$ & 0.05 & $7.34 \pm 0.7$ & $0.0423(0)$ & $4.534(5) \times 10^{37}$ \\
\hline 792 & 58738.966470614 & $563.7(4)$ & 1.95 ( 2 ) & 0.1 & $17.2 \pm 1.5$ & $0.0335(0)$ & $3.590(3) \times 10^{37}$ \\
\hline 793 & 58738.967428800 & $567.1(4)$ & $8.61(9)$ & 0.1 & $4.53 \pm 0.6$ & $0.0390(1)$ & $4.175(10) \times 10^{37}$ \\
\hline 794 & 58738.968358882 & $563.6(3)$ & $2.91(6)$ & 0.2 & $13.29 \pm 3.1$ & $0.0387(2)$ & $4.140(18) \times 10^{37}$ \\
\hline 795 & 58738.968358914 & $565.8(6)$ & $6.79(9)$ & 0.2 & $5.99 \pm 1.7$ & $0.0407(3)$ & $4.354(31) \times 10^{37}$ \\
\hline 796 & 58738.968768599 & $565.1(4)$ & $6.79(7)$ & 0.05 & $9.12 \pm 0.7$ & $0.0619(1)$ & $6.629(5) \times 10^{37}$ \\
\hline 797 & 58738.970772520 & $568.5(8)$ & $3.45(5)$ & 0.05 & $7.7 \pm 0.9$ & $0.0266(0)$ & $2.844(4) \times 10^{37}$ \\
\hline 798 & 58738.971650408 & $566.6(3)$ & $5.96(9)$ & 0.05 & $6.26 \pm 0.8$ & $0.0373(1)$ & $3.994(6) \times 10^{37}$ \\
\hline 799 & 58738.971906495 & $566.1(4)$ & $5.4(9)$ & 0.15 & $4.73 \pm 0.8$ & $0.0255(1)$ & $2.734(9) \times 10^{37}$ \\
\hline 800 & 58738.973060545 & $568.3(8)$ & $5.4(3)$ & 0.15 & $15.89 \pm 0.7$ & $0.0858(0)$ & $9.185(2) \times 10^{37}$ \\
\hline 801 & 58738.973109634 & $567.3(4)$ & $12.82(9)$ & 0.2 & $4.86 \pm 0.5$ & $0.0623(1)$ & $6.670(10) \times 10^{37}$ \\
\hline 802 & 58738.973310933 & 564.7 ( 3 ) & $5.88(9)$ & 0.05 & $5.15 \pm 0.7$ & $0.0303(1)$ & $3.242(7) \times 10^{37}$ \\
\hline 803 & 58738.974092719 & $569.8(5)$ & $3.71(5)$ & 0.15 & $2.73 \pm 0.0$ & $0.0101(0)$ & $1.086(0) \times 10^{37}$ \\
\hline 804 & 58738.974207371 & $565.2(6)$ & $3.39(4)$ & 0.4 & $17.44 \pm 1.0$ & $0.0591(0)$ & $6.329(3) \times 10^{37}$ \\
\hline 805 & 58738.974207416 & 564.7 ( 6 ) & $2.8(8)$ & 0.4 & $6.17 \pm 1.1$ & $0.0173(1)$ & $1.849(9) \times 10^{37}$ \\
\hline 806 & 58738.974207585 & $566.1(4)$ & $2.24(2)$ & 0.4 & $12.79 \pm 1.1$ & $0.0286(0)$ & $3.067(2) \times 10^{37}$ \\
\hline 807 & 58738.974354017 & $564.8(5)$ & $4.06(5)$ & 0.2 & $7.92 \pm 0.9$ & $0.0322(0)$ & $3.442(5) \times 10^{37}$ \\
\hline 808 & 58738.976248909 & $564.9(4)$ & 1.91 ( 3 ) & 0.05 & $10.44 \pm 1.2$ & $0.0199(0)$ & $2.135(3) \times 10^{37}$ \\
\hline 809 & 58738.976686641 & $563.8(3)$ & $2.84(4)$ & 0.05 & $7.9 \pm 1.0$ & $0.0224(0)$ & $2.402(4) \times 10^{37}$ \\
\hline 810 & 58738.977426143 & $566.1(6)$ & $5.51(6)$ & 0.05 & $9.3 \pm 0.8$ & $0.0512(0)$ & $5.485(5) \times 10^{37}$ \\
\hline 811 & 58738.977809236 & $565.5(6)$ & $0.5(2)$ & 0.1 & $9.5 \pm 1.2$ & $0.0047(0)$ & $5.085(26) \times 10^{36}$ \\
\hline 812 & 58738.978531047 & $567.6(6)$ & $5.09(4)$ & 0.15 & $10.28 \pm 0.7$ & $0.0523(0)$ & $5.601(3) \times 10^{37}$ \\
\hline 813 & 58738.978700101 & $566.4(7)$ & $5.32(4)$ & 0.15 & $14.06 \pm 0.8$ & $0.0748(0)$ & $8.007(3) \times 10^{37}$ \\
\hline
\end{tabular}


Table 1:

\begin{tabular}{|c|c|c|c|c|c|c|c|}
\hline Burst ID & $\begin{array}{c}\text { Burst time }^{a)} \\
\text { (MJD) }\end{array}$ & $\begin{array}{c}\mathrm{DM} \\
\left(\mathrm{pc} \mathrm{cm}^{-3}\right)\end{array}$ & $\begin{array}{l}\text { Width } \\
(\mathrm{ms})\end{array}$ & $\begin{array}{c}\text { Bandwidth }^{b)} \\
(\mathrm{GHz})\end{array}$ & $\begin{array}{c}\text { Peak Flux } \\
(\mathrm{mJy})\end{array}$ & $\begin{array}{c}\text { Fluence } \\
\text { (Jy ms) }\end{array}$ & $\begin{array}{c}\text { Energy } \\
\text { (erg) }\end{array}$ \\
\hline 814 & 58738.979018682 & $564.7(6)$ & $7.46(9)$ & 0.2 & $4.78 \pm 0.7$ & $0.0357(1)$ & $3.817(10) \times 10^{37}$ \\
\hline 815 & 58738.981353825 & $567.9(9)$ & $3.63(3)$ & 0.1 & $14.96 \pm 0.9$ & $0.0543(0)$ & $5.813(2) \times 10^{37}$ \\
\hline 816 & 58738.983485585 & $563.7(3)$ & $5.54(9)$ & 0.1 & $5.37 \pm 0.8$ & $0.0297(1)$ & $3.185(7) \times 10^{37}$ \\
\hline 817 & 58738.983486450 & $565.5(6)$ & $3.19(5)$ & 0.15 & $12.58 \pm 1.0$ & $0.0401(0)$ & $4.296(5) \times 10^{37}$ \\
\hline 818 & 58738.983486491 & $566.5(8)$ & $2.52(3)$ & 0.15 & $16.17 \pm 1.2$ & $0.0408(0)$ & $4.362(3) \times 10^{37}$ \\
\hline 819 & 58738.985892978 & $561.9(5)$ & $7.05(9)$ & 0.12 & $5.127 \pm 0.0$ & $0.0362(0)$ & $3.870(3) \times 10^{37}$ \\
\hline 821 & 58738.987950018 & $566.4(7)$ & $1.97(1)$ & 0.1 & $22.91 \pm 1.2$ & $0.0451(0)$ & $4.831(1) \times 10^{37}$ \\
\hline 822 & 58738.987950035 & $567.4(6)$ & $3.45(6)$ & 0.07 & $12.91 \pm 2.4$ & $0.0445(1)$ & $4.768(14) \times 10^{37}$ \\
\hline 823 & 58738.988169778 & $565.5(4)$ & $8.47(9)$ & 0.15 & $5.45 \pm 0.6$ & $0.0462(1)$ & $4.942(6) \times 10^{37}$ \\
\hline 824 & 58738.988564430 & $566.1(5)$ & $4.44(7)$ & 0.15 & $5.75 \pm 0.7$ & $0.0255(0)$ & $2.733(5) \times 10^{37}$ \\
\hline 825 & 58738.988588558 & $567.0(8)$ & $2.65(1)$ & 0.3 & $22.56 \pm 1.0$ & $0.0598(0)$ & $6.400(1) \times 10^{37}$ \\
\hline 826 & 58738.988588580 & $567.2(6)$ & $1.1(5)$ & 0.11 & $5.51 \pm 0.9$ & $0.0061(0)$ & $6.488(48) \times 10^{36}$ \\
\hline 827 & 58738.988604877 & $568.4(6)$ & $4.37(3)$ & 0.3 & $15.52 \pm 0.8$ & $0.0678(0)$ & $7.260(2) \times 10^{37}$ \\
\hline 828 & 58738.989113269 & $566.4(3)$ & $2.46(5)$ & 0.2 & $7.3 \pm 1.2$ & $0.0180(1)$ & $1.922(5) \times 10^{37}$ \\
\hline 830 & 58738.990210552 & $569.3(7)$ & $6.05(9)$ & 0.05 & $7.09 \pm 0.9$ & $0.0429(1)$ & $4.592(8) \times 10^{37}$ \\
\hline 831 & 58738.991138562 & $568.9(8)$ & $14.43(9)$ & 0.1 & $4.83 \pm 0.6$ & $0.0697(1)$ & $7.461(15) \times 10^{37}$ \\
\hline 832 & 58738.991425582 & $569.3(10)$ & $7.82(6)$ & 0.2 & $10.06 \pm 0.6$ & $0.0787(0)$ & $8.421(3) \times 10^{37}$ \\
\hline 833 & 58738.993118070 & $566.1(12)$ & $1.59(2)$ & 0.15 & $15.5 \pm 1.4$ & $0.0246(0)$ & $2.638(2) \times 10^{37}$ \\
\hline 834 & 58738.993118119 & $565.5(10)$ & $1.12(1)$ & 0.12 & $16.6 \pm 0.3$ & $0.0186(0)$ & $1.990(0) \times 10^{37}$ \\
\hline 835 & 58738.994140846 & $568.7(6)$ & $9.09(9)$ & 0.1 & $6.19 \pm 0.6$ & $0.0563(1)$ & $6.023(7) \times 10^{37}$ \\
\hline 836 & 58738.995007935 & $566.7(5)$ & $3.69(5)$ & 0.1 & $4.574 \pm 0.0$ & $0.0169(0)$ & $1.806(1) \times 10^{37}$ \\
\hline 837 & 58738.996852673 & $567.2(5)$ & $7.84(9)$ & 0.1 & $3.855 \pm 0.0$ & $0.0302(0)$ & $3.235(3) \times 10^{37}$ \\
\hline 838 & 58738.997810547 & $566.4(4)$ & $3.19(3)$ & 0.25 & $13.85 \pm 0.9$ & $0.0442(0)$ & $4.730(2) \times 10^{37}$ \\
\hline 839 & 58738.997810628 & $563.8(6)$ & $4.54(5)$ & 0.25 & $9.53 \pm 0.8$ & $0.0433(0)$ & $4.632(3) \times 10^{37}$ \\
\hline 840 & 58741.878721653 & $565.7(3)$ & $2.82(5)$ & 0.4 & $10.89 \pm 1.5$ & 0.0307 ( 1 ) & $3.287(7) \times 10^{37}$ \\
\hline 841 & 58741.896348136 & $566.1(3)$ & $2.12(0)$ & 0.3 & $184.62 \pm 1.7$ & $0.3914(0)$ & $4.190(0) \times 10^{38}$ \\
\hline 842 & 58741.906732710 & $564.5(3)$ & $5.52(7)$ & 0.1 & $8.77 \pm 0.9$ & $0.0484(1)$ & $5.182(6) \times 10^{37}$ \\
\hline 843 & 58741.910679445 & $564.0(2)$ & $0.85(1)$ & 0.4 & $56.91 \pm 2.9$ & $0.0484(0)$ & $5.178(1) \times 10^{37}$ \\
\hline 844 & 58741.910679462 & $566.2(7)$ & $3.76(1)$ & 0.4 & $74.83 \pm 1.4$ & $0.2814(0)$ & $3.012(0) \times 10^{38}$ \\
\hline 845 & 58742.892325834 & $565.6(5)$ & $8.39(9)$ & 0.1 & $6.24 \pm 0.8$ & $0.0524(1)$ & $5.604(10) \times 10^{37}$ \\
\hline 848 & 58743.925098512 & $562.8(5)$ & $3.94(3)$ & 0.1 & $16.34 \pm 0.9$ & $0.0644(0)$ & $6.892(2) \times 10^{37}$ \\
\hline 849 & 58743.925818802 & $566.5(5)$ & $6.2(6)$ & 0.15 & $9.54 \pm 0.8$ & $0.0592(0)$ & $6.332(4) \times 10^{37}$ \\
\hline 850 & 58743.940609233 & $566.6(5)$ & $3.14(2)$ & 0.15 & $19.19 \pm 1.0$ & $0.0603(0)$ & $6.450(2) \times 10^{37}$ \\
\hline 851 & 58743.942644605 & $564.1(7)$ & $4.1(5)$ & 0.4 & $16.28 \pm 1.1$ & $0.0668(0)$ & $7.145(5) \times 10^{37}$ \\
\hline 852 & 58744.875032122 & $566.0(5)$ & $4.77(7)$ & 0.14 & $13.287 \pm 0.2$ & $0.0633(1)$ & $6.779(9) \times 10^{37}$ \\
\hline 853 & 58744.884265650 & $566.0(5)$ & $3.92(6)$ & 0.12 & $6.305 \pm 0.1$ & $0.0247(0)$ & $2.647(3) \times 10^{37}$ \\
\hline 854 & 58744.896132117 & $566.0(5)$ & $2.84(4)$ & 0.08 & $20.801 \pm 0.2$ & $0.0591(1)$ & $6.326(7) \times 10^{37}$ \\
\hline 855 & 58744.902384513 & $566.3(5)$ & $6.58(9)$ & 0.34 & $9.068 \pm 0.1$ & $0.0597(1)$ & $6.386(6) \times 10^{37}$ \\
\hline 856 & 58745.926172894 & $565.4(5)$ & $4.84(4)$ & 0.2 & $11.71 \pm 0.8$ & $0.0567(0)$ & $6.067(2) \times 10^{37}$ \\
\hline 857 & 58745.926172925 & $565.8(6)$ & $6.1(9)$ & 0.13 & $5.31 \pm 0.9$ & $0.0324(1)$ & $3.467(11) \times 10^{37}$ \\
\hline 858 & 58745.927117966 & $565.6(6)$ & $3.25(9)$ & 0.2 & $4.98 \pm 1.6$ & $0.0162(2)$ & $1.733(19) \times 10^{37}$ \\
\hline 859 & 58745.927118030 & $565.6(5)$ & $11.17(9)$ & 0.25 & $8.38 \pm 0.7$ & $0.0936(1)$ & $1.002(1) \times 10^{38}$ \\
\hline 860 & 58745.927924896 & $564.8(4)$ & $4.2(3)$ & 0.08 & $15.95 \pm 0.9$ & $0.0670(0)$ & $7.171(2) \times 10^{37}$ \\
\hline 861 & 58745.927924914 & $568.7(10)$ & $5.56(9)$ & 0.18 & $5.01 \pm 0.7$ & $0.0279(1)$ & $2.982(9) \times 10^{37}$ \\
\hline 862 & 58745.930735422 & $566.7(6)$ & $5.03(6)$ & 0.15 & $8.19 \pm 0.8$ & $0.0412(1)$ & $4.410(5) \times 10^{37}$ \\
\hline 863 & 58745.930868265 & $567.7(5)$ & $4.22(6)$ & 0.05 & $8.05 \pm 0.9$ & $0.0340(1)$ & $3.637(5) \times 10^{37}$ \\
\hline 864 & 58745.930975561 & $568.5(5)$ & $4.46(6)$ & 0.18 & $6.672 \pm 0.1$ & $0.0298(0)$ & $3.187(2) \times 10^{37}$ \\
\hline 865 & 58745.933259008 & $566.4(5)$ & $6.4(8)$ & 0.1 & $6.88 \pm 0.7$ & $0.0440(1)$ & $4.714(5) \times 10^{37}$ \\
\hline 866 & 58745.934533431 & $566.7(8)$ & $3.52(5)$ & 0.1 & $7.43 \pm 1.0$ & $0.0261(1)$ & $2.800(5) \times 10^{37}$ \\
\hline 867 & 58745.935009556 & $569.8(5)$ & $7.49(9)$ & 0.17 & $4.151 \pm 0.0$ & $0.0311(0)$ & $3.327(2) \times 10^{37}$ \\
\hline 868 & 58745.937603269 & $566.0(5)$ & $3.72(4)$ & 0.1 & $9.65 \pm 0.9$ & $0.0359(0)$ & $3.843(3) \times 10^{37}$ \\
\hline 869 & 58745.939232967 & $568.2(7)$ & $6.41(9)$ & 0.2 & $5.77 \pm 0.7$ & $0.0370(1)$ & $3.959(7) \times 10^{37}$ \\
\hline 870 & 58745.940414050 & $569.0(5)$ & $3.25(5)$ & 0.1 & $7.48 \pm 1.0$ & $0.0243(0)$ & $2.602(5) \times 10^{37}$ \\
\hline 871 & 58745.941749435 & $566.2(4)$ & $5.21(6)$ & 0.1 & $8.95 \pm 0.8$ & $0.0466(0)$ & $4.992(5) \times 10^{37}$ \\
\hline 872 & 58745.943742922 & $566.4(7)$ & $3.9(5)$ & 0.2 & $8.72 \pm 0.9$ & $0.0340(0)$ & $3.641(5) \times 10^{37}$ \\
\hline 873 & 58745.943743782 & $563.9(5)$ & $6.06(5)$ & 0.2 & $11.27 \pm 0.8$ & $0.0683(0)$ & $7.311(4) \times 10^{37}$ \\
\hline 874 & 58745.943964729 & $568.2(7)$ & $9.99(9)$ & 0.1 & $5.2 \pm 0.6$ & $0.0520(1)$ & $5.561(9) \times 10^{37}$ \\
\hline 875 & 58745.945355618 & $563.4(6)$ & $2.66(2)$ & 0.06 & $21.65 \pm 1.1$ & $0.0576(0)$ & $6.165(2) \times 10^{37}$ \\
\hline 876 & 58745.945703413 & $563.3(8)$ & $5.86(5)$ & 0.22 & $9.49 \pm 0.7$ & $0.0556(0)$ & $5.953(4) \times 10^{37}$ \\
\hline
\end{tabular}


Table 1:

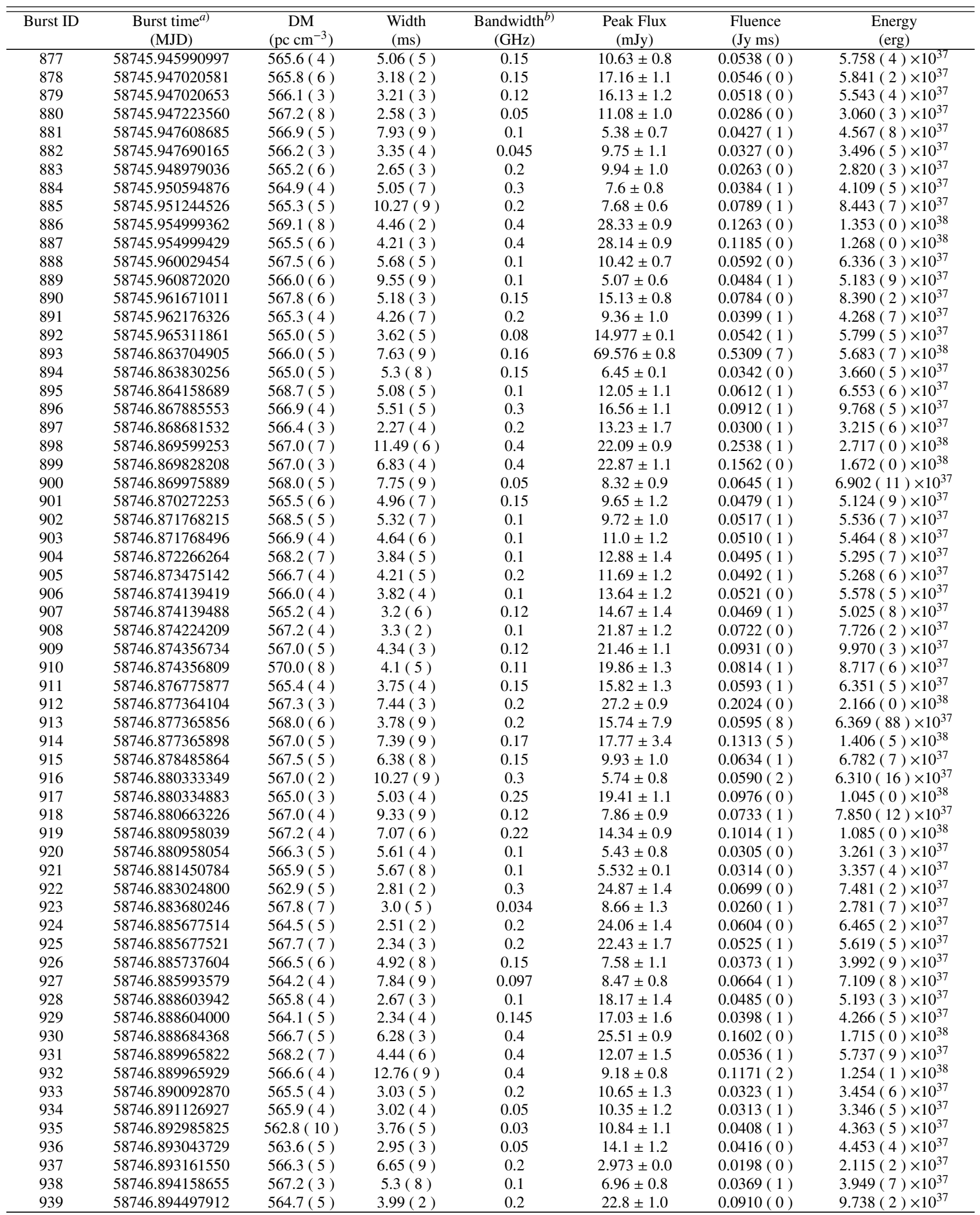


Table 1:

\begin{tabular}{|c|c|c|c|c|c|c|c|}
\hline Burst ID & $\begin{array}{l}\text { Burst time }^{a)} \\
\text { (MJD) }\end{array}$ & $\begin{array}{c}\mathrm{DM} \\
\left(\mathrm{pc} \mathrm{cm}^{-3}\right)\end{array}$ & $\begin{array}{l}\text { Width } \\
\text { (ms) }\end{array}$ & $\begin{array}{c}\text { Bandwidth }^{b)} \\
(\mathrm{GHz})\end{array}$ & $\begin{array}{c}\text { Peak Flux } \\
(\mathrm{mJy})\end{array}$ & $\begin{array}{c}\begin{array}{c}\text { Fluence } \\
(\mathrm{Jy} \mathrm{ms})\end{array} \\
\end{array}$ & $\begin{array}{c}\text { Energy } \\
\text { (erg) }\end{array}$ \\
\hline 940 & 58746.894497975 & $566.9(3)$ & $2.1(4)$ & 0.18 & $18.76 \pm 1.3$ & $0.0394(0)$ & $4.217(4) \times 10^{37}$ \\
\hline 941 & 58746.895918159 & $565.3(10)$ & $3.3(6)$ & 0.2 & $12.92 \pm 2.1$ & $0.0426(1)$ & $4.564(12) \times 10^{37}$ \\
\hline 942 & 58746.895918218 & 566.4 ( 3 ) & $9.97(9)$ & 0.3 & $10.52 \pm 0.8$ & $0.1049(1)$ & $1.123(1) \times 10^{38}$ \\
\hline 943 & 58746.895947041 & $565.3(6)$ & $2.58(1)$ & 0.3 & $61.11 \pm 1.4$ & $0.1577(0)$ & $1.688(0) \times 10^{38}$ \\
\hline 944 & 58746.902567494 & $566.0(5)$ & $3.83(6)$ & 0.21 & $20.145 \pm 0.2$ & $0.0772(1)$ & $8.267(8) \times 10^{37}$ \\
\hline 945 & 58747.844561099 & $565.8(4)$ & $7.47(9)$ & 0.05 & $10.03 \pm 0.9$ & $0.0749(1)$ & $8.020(8) \times 10^{37}$ \\
\hline 946 & 58747.845633767 & $566.4(4)$ & $7.84(9)$ & 0.3 & $7.83 \pm 0.8$ & $0.0614(1)$ & $6.571(10) \times 10^{37}$ \\
\hline 947 & 58747.846000743 & $564.9(5)$ & $9.59(9)$ & 0.2 & $5.81 \pm 0.8$ & $0.0557(1)$ & $5.965(13) \times 10^{37}$ \\
\hline 948 & 58747.846408306 & $570.0(4)$ & $4.8(8)$ & 0.1 & $7.78 \pm 1.1$ & $0.0373(1)$ & $3.998(8) \times 10^{37}$ \\
\hline 949 & 58747.848509833 & $565.0(6)$ & $3.52(2)$ & 0.1 & $21.12 \pm 1.2$ & $0.0743(0)$ & $7.958(3) \times 10^{37}$ \\
\hline 950 & 58747.851778271 & $567.0(8)$ & $2.42(0)$ & 0.3 & $896.24 \pm 5.0$ & $2.1689(0)$ & $2.322(0) \times 10^{39}$ \\
\hline 951 & 58747.852664508 & $565.2(3)$ & 3.67 ( 5 ) & 0.1 & $11.39 \pm 1.3$ & $0.0418(1)$ & $4.475(7) \times 10^{37}$ \\
\hline 952 & 58747.852987546 & $567.1(6)$ & $2.87(4)$ & 0.07 & $13.23 \pm 1.6$ & $0.0380(1)$ & $4.065(6) \times 10^{37}$ \\
\hline 953 & 58747.855606907 & $567.4(6)$ & 3.05 ( 3 ) & 0.08 & $17.21 \pm 1.3$ & $0.0525(0)$ & $5.619(3) \times 10^{37}$ \\
\hline 954 & 58747.855848964 & $565.4(4)$ & $7.75(9)$ & 0.05 & $6.64 \pm 0.8$ & $0.0515(1)$ & $5.509(10) \times 10^{37}$ \\
\hline 955 & 58747.860001941 & $568.8(5)$ & $5.79(7)$ & 0.05 & $9.94 \pm 1.0$ & $0.0575(1)$ & $6.161(7) \times 10^{37}$ \\
\hline 956 & 58747.860773095 & $565.4(4)$ & $4.49(5)$ & 0.2 & $12.46 \pm 1.1$ & $0.0559(0)$ & $5.989(5) \times 10^{37}$ \\
\hline 957 & 58747.862167043 & $565.8(5)$ & $2.32(4)$ & 0.15 & $10.34 \pm 1.6$ & $0.0240(1)$ & $2.568(7) \times 10^{37}$ \\
\hline 958 & 58747.863486680 & $567.0(6)$ & $3.84(5)$ & 0.07 & $11.75 \pm 1.3$ & $0.0451(1)$ & $4.830(6) \times 10^{37}$ \\
\hline 959 & 58747.863486702 & $566.6(8)$ & $3.11(5)$ & 0.1 & $10.01 \pm 1.2$ & $0.0311(1)$ & $3.333(6) \times 10^{37}$ \\
\hline 960 & 58747.863746228 & $564.5(5)$ & $2.6(1)$ & 0.21 & $37.8 \pm 1.4$ & $0.0983(0)$ & $1.052(0) \times 10^{38}$ \\
\hline 961 & 58747.863746234 & $564.0(4)$ & $2.3(2)$ & 0.21 & $34.69 \pm 1.5$ & $0.0798(0)$ & $8.541(3) \times 10^{37}$ \\
\hline 962 & 58747.864719127 & $565.6(6)$ & $6.74(7)$ & 0.17 & $11.41 \pm 1.0$ & $0.0769(1)$ & $8.232(7) \times 10^{37}$ \\
\hline 963 & 58747.865709419 & $564.8(5)$ & $4.73(9)$ & 0.185 & $6.81 \pm 1.1$ & $0.0322(1)$ & $3.448(9) \times 10^{37}$ \\
\hline 964 & 58747.866733239 & 566.7 ( 5 ) & $4.03(8)$ & 0.15 & $8.53 \pm 1.3$ & $0.0344(1)$ & $3.680(10) \times 10^{37}$ \\
\hline 965 & 58747.867068664 & 568.7 ( 6$)$ & $2.61(4)$ & 0.1 & $10.82 \pm 1.3$ & $0.0282(0)$ & $3.023(5) \times 10^{37}$ \\
\hline 966 & 58748.912227745 & $566.0(5)$ & $3.59(5)$ & 0.15 & $18.456 \pm 0.1$ & $0.0663(1)$ & $7.097(6) \times 10^{37}$ \\
\hline 967 & 58748.912813535 & $566.0(5)$ & 4.17 ( 6 ) & 0.17 & $37.236 \pm 0.3$ & $0.1554(1)$ & $1.664(1) \times 10^{38}$ \\
\hline 968 & 58748.914438871 & $564.1(5)$ & $2.78(4)$ & 0.14 & $8.804 \pm 0.1$ & $0.0244(0)$ & $2.617(2) \times 10^{37}$ \\
\hline 969 & 58748.915072066 & $564.2(4)$ & $4.91(5)$ & 0.15 & $9.66 \pm 0.8$ & $0.0474(0)$ & $5.077(4) \times 10^{37}$ \\
\hline 970 & 58748.915072710 & $567.0(5)$ & $2.87(5)$ & 0.12 & $7.13 \pm 1.0$ & $0.0205(0)$ & $2.191(5) \times 10^{37}$ \\
\hline 971 & 58748.916527244 & $567.0(12)$ & $8.91(9)$ & 0.14 & $8.62 \pm 0.7$ & $0.0768(1)$ & $8.222(6) \times 10^{37}$ \\
\hline 972 & 58748.916561163 & $565.6(6)$ & $2.01(4)$ & 0.4 & $10.6 \pm 1.6$ & $0.0213(1)$ & $2.281(6) \times 10^{37}$ \\
\hline 973 & 58748.916561175 & $565.7(4)$ & $9.04(7)$ & 0.4 & $13.31 \pm 1.2$ & $0.1203(1)$ & $1.288(0) \times 10^{38}$ \\
\hline 974 & 58748.916565383 & $564.5(6)$ & $6.89(9)$ & 0.4 & $5.29 \pm 0.8$ & $0.0365(1)$ & $3.902(9) \times 10^{37}$ \\
\hline 975 & 58748.918503295 & $567.1(7)$ & $4.44(3)$ & 0.15 & $14.53 \pm 0.8$ & $0.0645(0)$ & $6.906(2) \times 10^{37}$ \\
\hline 976 & 58748.918503299 & $566.0(4)$ & $5.21(5)$ & 0.05 & $4.29 \pm 1.2$ & $0.0223(1)$ & $2.393(6) \times 10^{37}$ \\
\hline 977 & 58748.919463421 & $566.7(5)$ & $0.78(3)$ & 0.25 & $5.96 \pm 2.0$ & $0.0046(1)$ & $4.976(71) \times 10^{36}$ \\
\hline 978 & 58748.919463446 & $563.9(7)$ & $3.57(7)$ & 0.1 & $7.13 \pm 0.8$ & $0.0255(1)$ & $2.725(5) \times 10^{37}$ \\
\hline 979 & 58748.922247994 & $567.0(3)$ & $3.65(7)$ & 0.1 & $6.28 \pm 1.0$ & $0.0229(1)$ & $2.454(7) \times 10^{37}$ \\
\hline 980 & 58748.924422443 & $566.1(5)$ & $2.41(6)$ & 0.4 & $5.21 \pm 1.1$ & $0.0126(1)$ & $1.344(6) \times 10^{37}$ \\
\hline 981 & 58748.925020254 & $566.5(5)$ & $2.21(9)$ & 0.4 & $3.3 \pm 1.7$ & $0.0073(2)$ & $7.807(231) \times 10^{36}$ \\
\hline 982 & 58748.925020301 & 567.0 ( 3 ) & $5.38(8)$ & 0.4 & $10.39 \pm 0.8$ & $0.0559(1)$ & $5.984(7) \times 10^{37}$ \\
\hline 983 & 58748.925020502 & $566.9(3)$ & $8.68(9)$ & 0.4 & $6.19 \pm 0.6$ & $0.0537(1)$ & $5.752(7) \times 10^{37}$ \\
\hline 984 & 58748.925665405 & $565.3(6)$ & $5.36(9)$ & 0.15 & $5.24 \pm 0.8$ & $0.0281(1)$ & $3.007(7) \times 10^{37}$ \\
\hline 985 & 58748.926648222 & $567.2(8)$ & $6.31(9)$ & 0.16 & $6.61 \pm 0.8$ & $0.0417(1)$ & $4.465(7) \times 10^{37}$ \\
\hline 986 & 58748.927545205 & $566.2(7)$ & 4.19 ( 3 ) & 0.19 & $15.44 \pm 0.9$ & $0.0647(0)$ & $6.925(2) \times 10^{37}$ \\
\hline 987 & 58748.927545272 & $566.6(3)$ & $4.08(5)$ & 0.25 & $18.48 \pm 1.1$ & $0.0754(1)$ & $8.071(5) \times 10^{37}$ \\
\hline 988 & 58748.927831214 & $565.3(4)$ & $4.78(7)$ & 0.1 & $5.9 \pm 0.7$ & $0.0282(1)$ & $3.019(5) \times 10^{37}$ \\
\hline 989 & 58748.927831873 & $564.5(5)$ & $2.4(3)$ & 0.3 & $10.87 \pm 1.0$ & $0.0261(0)$ & $2.793(2) \times 10^{37}$ \\
\hline 990 & 58748.929596378 & $565.8(5)$ & $3.1(1)$ & 0.4 & $134.22 \pm 1.3$ & $0.4161(0)$ & $4.454(0) \times 10^{38}$ \\
\hline 991 & 58748.930320758 & $564.9(4)$ & $2.47(4)$ & 0.1 & $8.27 \pm 1.0$ & $0.0204(0)$ & $2.187(3) \times 10^{37}$ \\
\hline 992 & 58748.930528970 & $566.3(5)$ & $3.2(5)$ & 0.15 & $2.566 \pm 0.0$ & $0.0082(0)$ & $8.787(7) \times 10^{36}$ \\
\hline 993 & 58748.930822123 & $565.0(6)$ & $4.22(3)$ & 0.15 & $16.31 \pm 0.8$ & $0.0688(0)$ & $7.368(2) \times 10^{37}$ \\
\hline 994 & 58748.931118508 & $563.5(6)$ & $3.95(2)$ & 0.18 & $23.88 \pm 1.1$ & $0.0943(0)$ & $1.010(0) \times 10^{38}$ \\
\hline 995 & 58748.931118544 & $566.3(6)$ & $16.42(9)$ & 0.18 & $9.67 \pm 0.9$ & $0.1588(2)$ & $1.700(1) \times 10^{38}$ \\
\hline 996 & 58748.931655473 & $569.4(7)$ & $4.88(6)$ & 0.2 & $8.43 \pm 0.8$ & $0.0411(0)$ & $4.404(4) \times 10^{37}$ \\
\hline 997 & 58748.932075230 & $566.3(4)$ & $17.55(9)$ & 0.05 & $4.43 \pm 0.6$ & $0.0777(2)$ & $8.323(24) \times 10^{37}$ \\
\hline 998 & 58748.932115398 & $567.0(3)$ & $9.76(9)$ & 0.12 & $5.18 \pm 0.6$ & $0.0506(1)$ & $5.412(8) \times 10^{37}$ \\
\hline 999 & 58748.933152326 & $565.7(5)$ & $16.24(9)$ & 0.4 & $4.13 \pm 0.6$ & $0.0671(2)$ & $7.180(19) \times 10^{37}$ \\
\hline 1000 & 58748.933650035 & $567.7(7)$ & $3.51(3)$ & 0.2 & $13.12 \pm 0.9$ & $0.0461(0)$ & $4.930(2) \times 10^{37}$ \\
\hline 1001 & 58748.933650064 & $568.7(6)$ & $1.34(4)$ & 0.1 & $4.09 \pm 0.7$ & $0.0055(0)$ & $5.867(27) \times 10^{36}$ \\
\hline 1002 & 58748.934887599 & $565.5(6)$ & $3.77(6)$ & 0.15 & $12.18 \pm 1.0$ & $0.0459(1)$ & $4.916(5) \times 10^{37}$ \\
\hline
\end{tabular}


Table 1:

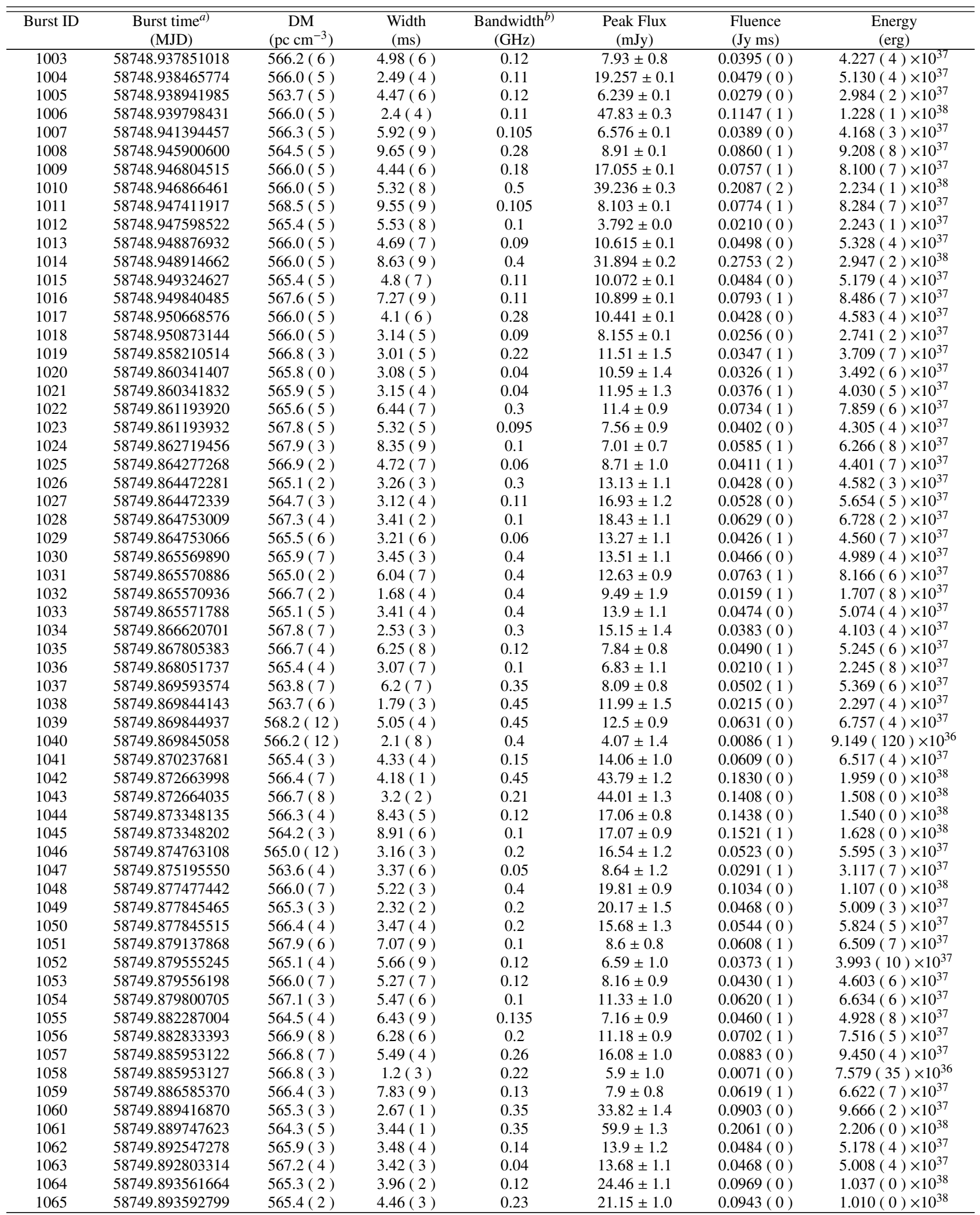


Table 1:

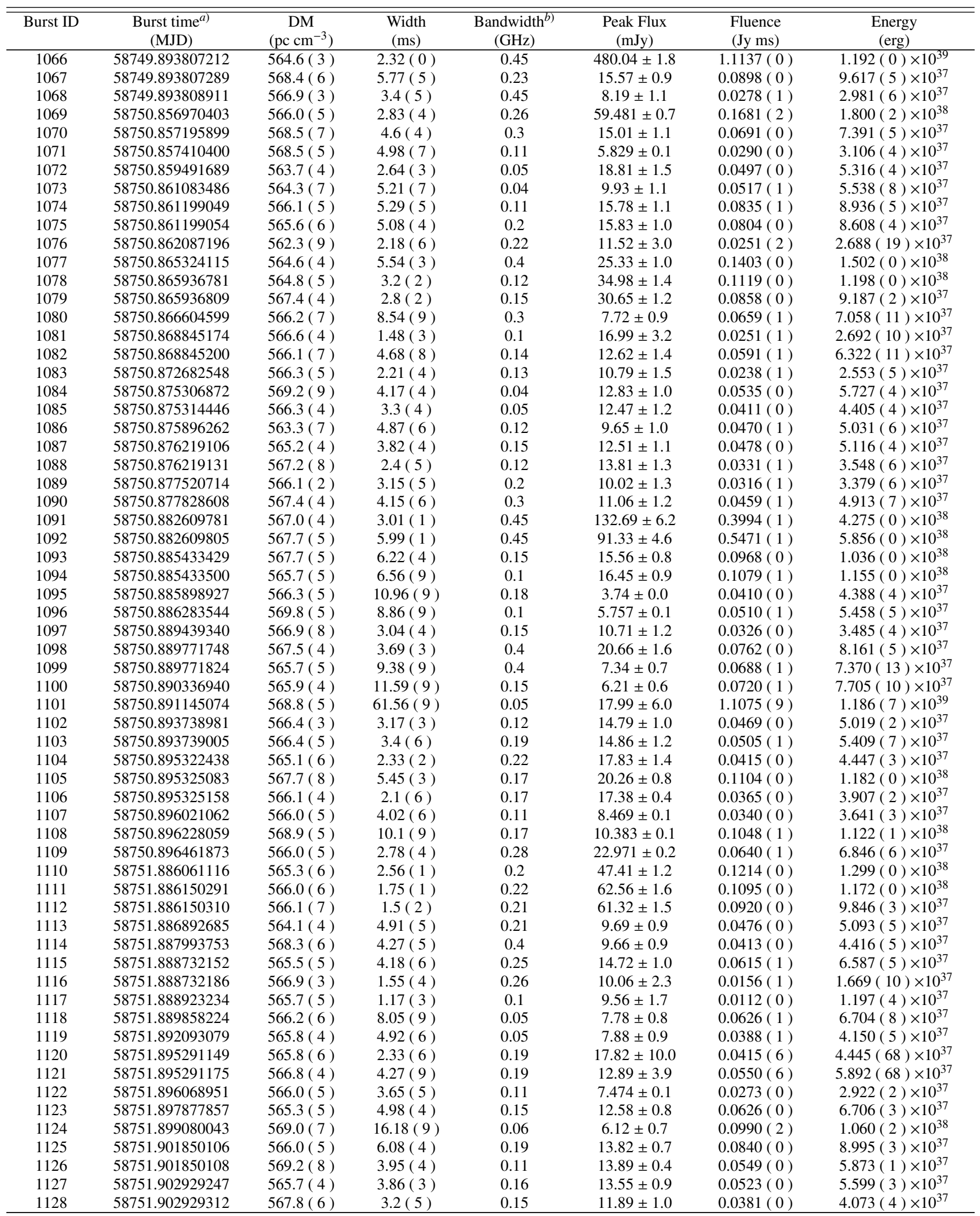


Table 1:

\begin{tabular}{|c|c|c|c|c|c|c|c|}
\hline Burst ID & $\begin{array}{c}\text { Burst time }^{a)} \\
\text { (MJD) }\end{array}$ & $\begin{array}{c}\mathrm{DM} \\
\left(\mathrm{pc} \mathrm{cm}^{-3}\right)\end{array}$ & $\begin{array}{l}\text { Width } \\
(\mathrm{ms})\end{array}$ & $\begin{array}{c}\left.\text { Bandwidth }^{b}\right) \\
(\mathrm{GHz})\end{array}$ & $\begin{array}{c}\text { Peak Flux } \\
(\mathrm{mJy})\end{array}$ & $\begin{array}{c}\text { Fluence } \\
\text { (Jy ms) }\end{array}$ & $\begin{array}{c}\text { Energy } \\
\text { (erg) }\end{array}$ \\
\hline 1129 & 58751.904052686 & $566.0(4)$ & $4.5(4)$ & 0.1 & $10.0 \pm 0.8$ & $0.0450(0)$ & $4.817(3) \times 10^{37}$ \\
\hline 1130 & 58751.904230597 & $565.1(6)$ & $6.83(6)$ & 0.2 & $8.98 \pm 0.7$ & $0.0613(0)$ & $6.566(4) \times 10^{37}$ \\
\hline 1131 & 58751.904324392 & $567.2(8)$ & $2.43(8)$ & 0.1 & $5.25 \pm 1.3$ & $0.0128(1)$ & $1.366(10) \times 10^{37}$ \\
\hline 1132 & 58751.905181385 & $562.4(6)$ & $5.74(4)$ & 0.4 & $13.82 \pm 0.8$ & $0.0793(0)$ & $8.492(3) \times 10^{37}$ \\
\hline 1133 & 58751.907251453 & $566.9(4)$ & $3.44(6)$ & 0.1 & $6.69 \pm 0.9$ & $0.0230(1)$ & $2.464(5) \times 10^{37}$ \\
\hline 1134 & 58751.907359175 & $566.0(5)$ & $6.15(7)$ & 0.05 & $7.23 \pm 0.7$ & $0.0445(0)$ & $4.760(5) \times 10^{37}$ \\
\hline 1136 & 58751.910802356 & $564.4(5)$ & $3.36(3)$ & 0.1 & $11.86 \pm 0.9$ & $0.0398(0)$ & $4.266(2) \times 10^{37}$ \\
\hline 1137 & 58751.911106877 & $564.4(4)$ & $4.41(6)$ & 0.05 & $6.4 \pm 0.8$ & $0.0282(0)$ & $3.021(5) \times 10^{37}$ \\
\hline 1138 & 58751.913138180 & $566.2(5)$ & $1.97(1)$ & 0.1 & $23.13 \pm 1.2$ & $0.0456(0)$ & $4.878(1) \times 10^{37}$ \\
\hline 1139 & 58751.913168389 & $568.5(9)$ & $6.82(5)$ & 0.16 & $11.71 \pm 0.7$ & $0.0799(0)$ & $8.549(3) \times 10^{37}$ \\
\hline 1140 & 58751.913168401 & $565.5(12)$ & $4.8(5)$ & 0.15 & $16.23 \pm 0.6$ & $0.0779(0)$ & $8.339(3) \times 10^{37}$ \\
\hline 1141 & 58751.913565924 & $567.8(3)$ & $3.03(3)$ & 0.04 & $13.74 \pm 1.1$ & $0.0416(0)$ & $4.457(3) \times 10^{37}$ \\
\hline 1142 & 58751.913776590 & $564.5(5)$ & $3.57(5)$ & 0.4 & $11.7 \pm 1.6$ & $0.0418(1)$ & $4.471(9) \times 10^{37}$ \\
\hline 1143 & 58751.914470328 & $566.9(3)$ & $3.82(7)$ & 0.13 & $5.64 \pm 0.8$ & $0.0215(1)$ & $2.306(6) \times 10^{37}$ \\
\hline 1145 & 58751.917680922 & $565.5(6)$ & $14.64(9)$ & 0.36 & $4.73 \pm 0.5$ & $0.0693(1)$ & $7.413(11) \times 10^{37}$ \\
\hline 1146 & 58751.917745156 & $562.9(7)$ & $3.1(4)$ & 0.1 & $7.52 \pm 0.9$ & $0.0233(0)$ & $2.496(4) \times 10^{37}$ \\
\hline 1147 & 58751.919463323 & $566.1(4)$ & $6.39(6)$ & 0.13 & $7.52 \pm 0.6$ & $0.0481(0)$ & $5.144(4) \times 10^{37}$ \\
\hline 1148 & 58751.919614615 & $570.0(6)$ & $4.54(4)$ & 0.15 & $11.95 \pm 0.8$ & $0.0542(0)$ & $5.808(3) \times 10^{37}$ \\
\hline 1149 & 58751.919902150 & $568.8(7)$ & $4.51(2)$ & 0.3 & $17.55 \pm 0.8$ & $0.0791(0)$ & $8.473(2) \times 10^{37}$ \\
\hline 1150 & 58751.919902160 & $564.2(4)$ & $3.41(7)$ & 0.06 & $12.45 \pm 0.8$ & $0.0425(1)$ & $4.545(5) \times 10^{37}$ \\
\hline 1151 & 58751.920814646 & $565.1(5)$ & $5.94(7)$ & 0.3 & $6.95 \pm 0.7$ & $0.0413(0)$ & $4.419(4) \times 10^{37}$ \\
\hline 1152 & 58751.921799633 & $567.5(6)$ & $1.34(9)$ & 0.1 & $3.21 \pm 1.5$ & $0.0043(1)$ & $4.605(150) \times 10^{36}$ \\
\hline 1153 & 58751.921804108 & $566.5(6)$ & $2.47(9)$ & 0.12 & $3.57 \pm 1.2$ & $0.0088(1)$ & $9.439(139) \times 10^{36}$ \\
\hline 1154 & 58751.921804174 & $568.4(7)$ & $10.98(9)$ & 0.12 & $6.15 \pm 0.5$ & $0.0675(1)$ & $7.229(9) \times 10^{37}$ \\
\hline 1155 & 58752.850066487 & $566.0(5)$ & $3.21(5)$ & 0.11 & $13.391 \pm 0.2$ & $0.0430(1)$ & $4.608(6) \times 10^{37}$ \\
\hline 1156 & 58752.850890814 & $564.5(3)$ & $5.41(4)$ & 0.35 & $19.38 \pm 1.1$ & $0.1048(0)$ & $1.122(0) \times 10^{38}$ \\
\hline 1157 & 58752.850890877 & $565.5(5)$ & $3.21(4)$ & 0.305 & $15.4 \pm 1.0$ & $0.0494(0)$ & $5.292(4) \times 10^{37}$ \\
\hline 1158 & 58752.852098680 & $566.0(5)$ & $3.41(9)$ & 0.3 & $10.29 \pm 3.0$ & $0.0351(3)$ & $3.756(27) \times 10^{37}$ \\
\hline 1159 & 58752.852754210 & $569.0(5)$ & $8.51(9)$ & 0.1 & $7.586 \pm 0.1$ & $0.0646(1)$ & $6.914(9) \times 10^{37}$ \\
\hline 1160 & 58752.852832819 & $564.2(5)$ & $4.25(6)$ & 0.25 & $9.61 \pm 1.1$ & $0.0408(1)$ & $4.372(7) \times 10^{37}$ \\
\hline 1163 & 58752.853519176 & $565.3(4)$ & $3.62(2)$ & 0.32 & $30.52 \pm 1.3$ & $0.1105(0)$ & $1.183(0) \times 10^{38}$ \\
\hline 1164 & 58752.853611862 & $567.4(5)$ & $2.16(1)$ & 0.3 & $76.84 \pm 1.7$ & $0.1660(0)$ & $1.777(0) \times 10^{38}$ \\
\hline 1165 & 58752.854053638 & $564.1(6)$ & $3.63(3)$ & 0.06 & $16.49 \pm 1.3$ & $0.0599(0)$ & $6.408(4) \times 10^{37}$ \\
\hline 1166 & 58752.855229355 & $555.4(5)$ & $7.08(9)$ & 0.105 & $3.08 \pm 0.0$ & $0.0218(0)$ & $2.333(3) \times 10^{37}$ \\
\hline 1167 & 58752.855529045 & $567.2(10)$ & $6.24(2)$ & 0.45 & $37.61 \pm 1.0$ & $0.2347(0)$ & $2.512(0) \times 10^{38}$ \\
\hline 1168 & 58752.856016442 & $561.5(5)$ & $6.57(9)$ & 0.15 & $6.037 \pm 0.1$ & $0.0396(1)$ & $4.244(5) \times 10^{37}$ \\
\hline 1169 & 58752.856380724 & $567.4(6)$ & $5.01(4)$ & 0.3 & $18.13 \pm 1.1$ & $0.0908(0)$ & $9.723(4) \times 10^{37}$ \\
\hline 1170 & 58752.856622659 & $565.6(5)$ & $1.58(5)$ & 0.2 & $9.73 \pm 2.9$ & $0.0154(2)$ & $1.646(16) \times 10^{37}$ \\
\hline 1171 & 58752.856622689 & $569.0(7)$ & $5.11(9)$ & 0.2 & $10.91 \pm 1.2$ & $0.0558(1)$ & $5.968(11) \times 10^{37}$ \\
\hline 1172 & 58752.857616458 & $566.4(5)$ & $4.41(5)$ & 0.13 & $12.82 \pm 1.1$ & $0.0565(1)$ & $6.052(5) \times 10^{37}$ \\
\hline 1173 & 58752.859493919 & $565.1(3)$ & $7.98(9)$ & 0.05 & $7.31 \pm 0.8$ & $0.0583(1)$ & $6.245(8) \times 10^{37}$ \\
\hline 1174 & 58752.859569824 & $561.9(5)$ & $4.0(6)$ & 0.17 & $4.628 \pm 0.1$ & $0.0185(0)$ & $1.982(2) \times 10^{37}$ \\
\hline 1175 & 58752.859591869 & $567.1(2)$ & $6.42(6)$ & 0.22 & $10.8 \pm 0.9$ & $0.0693(1)$ & $7.422(5) \times 10^{37}$ \\
\hline 1176 & 58752.860348120 & $563.7(5)$ & $5.25(6)$ & 0.22 & $9.88 \pm 1.0$ & $0.0519(1)$ & $5.553(6) \times 10^{37}$ \\
\hline 1177 & 58752.862099329 & $566.6(8)$ & $6.27(8)$ & 0.2 & $8.77 \pm 0.9$ & $0.0550(1)$ & $5.886(7) \times 10^{37}$ \\
\hline 1178 & 58752.862715898 & $566.4(6)$ & $3.24(0)$ & 0.45 & $252.76 \pm 1.8$ & $0.8189(0)$ & $8.767(0) \times 10^{38}$ \\
\hline 1179 & 58752.862715973 & $567.7(4)$ & $5.12(1)$ & 0.45 & $90.75 \pm 1.5$ & $0.4646(0)$ & $4.974(0) \times 10^{38}$ \\
\hline 1180 & 58752.862716104 & $565.8(7)$ & $4.77(2)$ & 0.45 & $46.81 \pm 1.5$ & $0.2233(0)$ & $2.390(0) \times 10^{38}$ \\
\hline 1181 & 58752.863095467 & $567.1(5)$ & $4.77(4)$ & 0.45 & $17.27 \pm 1.1$ & $0.0824(0)$ & $8.818(5) \times 10^{37}$ \\
\hline 1182 & 58752.863095545 & $565.1(3)$ & $3.85(8)$ & 0.45 & $7.79 \pm 1.2$ & $0.0300(1)$ & $3.211(10) \times 10^{37}$ \\
\hline 1183 & 58752.863096753 & $567.5(5)$ & $6.26(9)$ & 0.45 & $13.96 \pm 3.0$ & $0.0874(3)$ & $9.355(30) \times 10^{37}$ \\
\hline 1184 & 58752.863096849 & $566.4(4)$ & $14.13(9)$ & 0.45 & $8.56 \pm 1.0$ & $0.1210(4)$ & $1.295(4) \times 10^{38}$ \\
\hline 1185 & 58752.865429661 & $562.8(5)$ & $5.11(7)$ & 0.17 & $5.503 \pm 0.1$ & $0.0281(0)$ & $3.012(3) \times 10^{37}$ \\
\hline 1186 & 58752.869664976 & $564.4(5)$ & $3.69(1)$ & 0.45 & $86.61 \pm 1.2$ & $0.3196(0)$ & $3.421(0) \times 10^{38}$ \\
\hline 1187 & 58752.869665049 & $564.7(5)$ & $3.57(3)$ & 0.45 & $71.14 \pm 5.6$ & $0.2540(2)$ & $2.719(1) \times 10^{38}$ \\
\hline 1188 & 58752.869665082 & $563.2(5)$ & $3.22(3)$ & 0.45 & $47.4 \pm 7.8$ & $0.1526(2)$ & $1.634(2) \times 10^{38}$ \\
\hline 1189 & 58752.870528164 & $567.3(12)$ & $4.59(3)$ & 0.25 & $15.08 \pm 0.9$ & $0.0692(0)$ & $7.410(2) \times 10^{37}$ \\
\hline 1190 & 58752.870649850 & $566.4(5)$ & $3.5(1)$ & 0.27 & $51.09 \pm 1.0$ & $0.1788(0)$ & $1.914(0) \times 10^{38}$ \\
\hline 1191 & 58752.873390940 & $567.4(5)$ & $4.71(4)$ & 0.2 & $11.45 \pm 0.8$ & $0.0539(0)$ & $5.773(3) \times 10^{37}$ \\
\hline
\end{tabular}


Table 1:

\begin{tabular}{|c|c|c|c|c|c|c|c|}
\hline Burst ID & $\begin{array}{l}\text { Burst time }^{a)} \\
\text { (MJD) }\end{array}$ & $\begin{array}{c}\mathrm{DM} \\
\left(\mathrm{pc} \mathrm{cm}^{-3}\right)\end{array}$ & $\begin{array}{l}\text { Width } \\
\text { (ms) }\end{array}$ & $\begin{array}{c}\text { Bandwidth }^{b)} \\
(\mathrm{GHz})\end{array}$ & $\begin{array}{c}\text { Peak Flux } \\
(\mathrm{mJy})\end{array}$ & $\begin{array}{c}\begin{array}{c}\text { Fluence } \\
(\mathrm{Jy} \mathrm{ms})\end{array} \\
\end{array}$ & $\begin{array}{c}\text { Energy } \\
\text { (erg) }\end{array}$ \\
\hline 1192 & 58752.873648703 & $565.9(3)$ & $1.37(1)$ & 0.1 & $20.34 \pm 1.4$ & $0.0279(0)$ & $2.983(1) \times 10^{37}$ \\
\hline 1193 & 58752.873789845 & $565.7(7)$ & $7.16(9)$ & 0.15 & $6.68 \pm 0.7$ & $0.0478(1)$ & $5.120(6) \times 10^{37}$ \\
\hline 1194 & 58752.874643178 & $563.7(5)$ & $0.43(2)$ & 0.05 & $8.65 \pm 2.7$ & $0.0037(0)$ & $3.982(45) \times 10^{36}$ \\
\hline 1195 & 58752.874643224 & $567.0(2)$ & $8.09(9)$ & 0.05 & $4.79 \pm 0.6$ & $0.0387(1)$ & $4.148(9) \times 10^{37}$ \\
\hline 1196 & 58752.875076921 & $566.0(3)$ & $3.48(5)$ & 0.15 & $8.37 \pm 1.0$ & $0.0291(1)$ & $3.118(5) \times 10^{37}$ \\
\hline 1197 & 58752.875077359 & $567.5(6)$ & $3.8(4)$ & 0.15 & $15.29 \pm 1.2$ & $0.0581(0)$ & $6.220(4) \times 10^{37}$ \\
\hline 1198 & 58752.875077578 & $567.3(3)$ & $4.63(6)$ & 0.3 & $10.8 \pm 1.2$ & $0.0500(1)$ & $5.353(7) \times 10^{37}$ \\
\hline 1199 & 58752.878861316 & $566.4(4)$ & $3.12(1)$ & 0.14 & $28.86 \pm 1.0$ & $0.0900(0)$ & $9.639(1) \times 10^{37}$ \\
\hline 1200 & 58752.879848125 & $564.4(3)$ & $2.22(5)$ & 0.14 & $12.4 \pm 1.4$ & $0.0275(1)$ & $2.947(7) \times 10^{37}$ \\
\hline 1201 & 58752.879848155 & $564.3(2)$ & $2.15(9)$ & 0.45 & $5.21 \pm 1.4$ & $0.0112(2)$ & $1.199(16) \times 10^{37}$ \\
\hline 1202 & 58752.881168217 & $568.0(6)$ & $3.11(1)$ & 0.45 & $40.04 \pm 1.1$ & $0.1245(0)$ & $1.333(0) \times 10^{38}$ \\
\hline 1203 & 58752.883093830 & $568.5(5)$ & $3.61(5)$ & 0.05 & $9.14 \pm 1.0$ & $0.0330(0)$ & $3.532(4) \times 10^{37}$ \\
\hline 1204 & 58752.884154121 & $565.2(6)$ & $4.48(5)$ & 0.05 & $9.32 \pm 0.9$ & $0.0418(1)$ & $4.470(5) \times 10^{37}$ \\
\hline 1205 & 58752.886664847 & $564.3(3)$ & $3.15(2)$ & 0.15 & $23.19 \pm 1.2$ & $0.0731(0)$ & $7.820(2) \times 10^{37}$ \\
\hline 1206 & 58752.886885480 & $564.4(6)$ & $2.6(3)$ & 0.22 & $10.93 \pm 1.2$ & $0.0284(0)$ & $3.042(4) \times 10^{37}$ \\
\hline 1207 & 58752.886990056 & $564.3(5)$ & $4.6(5)$ & 0.15 & $11.18 \pm 0.9$ & $0.0514(0)$ & $5.505(4) \times 10^{37}$ \\
\hline 1208 & 58752.888472990 & $563.4(3)$ & $9.9(9)$ & 0.4 & $6.92 \pm 0.7$ & $0.0685(1)$ & $7.334(9) \times 10^{37}$ \\
\hline 1209 & 58753.927697099 & $567.4(3)$ & $5.53(9)$ & 0.07 & $4.76 \pm 0.8$ & $0.0263(1)$ & $2.818(8) \times 10^{37}$ \\
\hline 1210 & 58753.928029614 & $565.2(3)$ & 3.41 ( 3 ) & 0.11 & $12.1 \pm 1.0$ & $0.0413(0)$ & $4.417(3) \times 10^{37}$ \\
\hline 1211 & 58753.928402208 & $565.6(5)$ & $5.82(6)$ & 0.4 & $8.74 \pm 0.8$ & $0.0509(0)$ & $5.445(5) \times 10^{37}$ \\
\hline 1212 & 58753.928402263 & $563.8(5)$ & $4.38(8)$ & 0.13 & $7.61 \pm 0.8$ & $0.0333(1)$ & $3.568(6) \times 10^{37}$ \\
\hline 1213 & 58753.929041225 & $564.5(6)$ & $2.74(3)$ & 0.14 & $11.89 \pm 1.1$ & $0.0326(0)$ & $3.487(3) \times 10^{37}$ \\
\hline 1214 & 58753.929041253 & $564.7(4)$ & $2.54(3)$ & 0.14 & $10.04 \pm 1.1$ & $0.0255(0)$ & $2.730(3) \times 10^{37}$ \\
\hline 1215 & 58753.929368055 & $566.3(5)$ & $3.04(0)$ & 0.45 & $558.92 \pm 1.4$ & $1.6991(0)$ & $1.819(0) \times 10^{39}$ \\
\hline 1216 & 58753.929368148 & $565.1(4)$ & $6.23(2)$ & 0.45 & $33.08 \pm 1.0$ & $0.2061(0)$ & $2.206(0) \times 10^{38}$ \\
\hline 1217 & 58753.929369222 & $564.6(5)$ & $4.37(2)$ & 0.45 & $20.44 \pm 0.9$ & $0.0893(0)$ & $9.562(2) \times 10^{37}$ \\
\hline 1218 & 58753.929369256 & $564.8(5)$ & $4.05(3)$ & 0.205 & $18.83 \pm 0.9$ & $0.0763(0)$ & $8.164(3) \times 10^{37}$ \\
\hline 1219 & 58753.929962987 & $566.9(6)$ & 3.97 ( 6 ) & 0.4 & $12.0 \pm 2.5$ & $0.0476(2)$ & $5.100(17) \times 10^{37}$ \\
\hline 1220 & 58753.930603042 & $567.1(4)$ & $3.92(4)$ & 0.2 & $10.35 \pm 1.0$ & $0.0406(0)$ & $4.343(4) \times 10^{37}$ \\
\hline 1221 & 58753.930689701 & $567.9(8)$ & $3.5(3)$ & 0.2 & $15.02 \pm 1.0$ & $0.0526(0)$ & $5.628(3) \times 10^{37}$ \\
\hline 1222 & 58753.932114294 & $566.1(7)$ & $5.0(8)$ & 0.15 & $5.79 \pm 0.7$ & $0.0290(1)$ & $3.099(5) \times 10^{37}$ \\
\hline 1223 & 58753.933387639 & $565.4(4)$ & $5.3(4)$ & 0.14 & $11.26 \pm 0.8$ & $0.0597(0)$ & $6.388(3) \times 10^{37}$ \\
\hline 1224 & 58753.933387641 & $564.7(6)$ & $4.9(7)$ & 0.14 & $8.93 \pm 0.8$ & $0.0438(1)$ & $4.684(5) \times 10^{37}$ \\
\hline 1225 & 58753.933767266 & 564.7 ( 3 ) & 3.39 ( 4$)$ & 0.16 & $8.72 \pm 0.9$ & $0.0296(0)$ & $3.164(4) \times 10^{37}$ \\
\hline 1226 & 58753.934058433 & $567.4(8)$ & $4.39(4)$ & 0.15 & $11.51 \pm 0.8$ & $0.0505(0)$ & $5.409(3) \times 10^{37}$ \\
\hline 1227 & 58753.934436630 & $569.3(12)$ & $3.28(4)$ & 0.31 & $8.9 \pm 0.9$ & $0.0292(0)$ & $3.125(4) \times 10^{37}$ \\
\hline 1228 & 58753.935640099 & $563.5(7)$ & $6.0(5)$ & 0.1 & $10.08 \pm 0.7$ & $0.0605(0)$ & $6.474(4) \times 10^{37}$ \\
\hline 1229 & 58753.935829568 & $565.1(4)$ & $7.13(7)$ & 0.45 & $8.43 \pm 0.6$ & $0.0601(0)$ & $6.434(4) \times 10^{37}$ \\
\hline 1230 & 58753.935830277 & $563.7(4)$ & $10.53(9)$ & 0.15 & $4.41 \pm 0.5$ & $0.0464(1)$ & $4.971(9) \times 10^{37}$ \\
\hline 1231 & 58753.936578439 & $567.8(3)$ & $2.87(1)$ & 0.16 & $70.43 \pm 1.1$ & $0.2021(0)$ & $2.164(0) \times 10^{38}$ \\
\hline 1232 & 58753.936681557 & $566.8(2)$ & $7.14(8)$ & 0.4 & $6.8 \pm 0.6$ & $0.0486(1)$ & $5.197(5) \times 10^{37}$ \\
\hline 1233 & 58753.937992698 & $567.7(8)$ & $3.26(5)$ & 0.12 & $7.25 \pm 0.9$ & $0.0236(0)$ & $2.530(4) \times 10^{37}$ \\
\hline 1234 & 58753.938511254 & $564.7(4)$ & $6.29(9)$ & 0.3 & $5.1 \pm 0.7$ & $0.0321(1)$ & $3.434(8) \times 10^{37}$ \\
\hline 1235 & 58753.939194735 & $565.5(4)$ & $4.29(3)$ & 0.09 & $13.33 \pm 0.8$ & $0.0572(0)$ & $6.122(2) \times 10^{37}$ \\
\hline 1236 & 58753.939194797 & $566.3(12)$ & $3.45(6)$ & 0.085 & $12.34 \pm 0.9$ & $0.0426(1)$ & $4.557(5) \times 10^{37}$ \\
\hline 1237 & 58753.939289805 & $567.8(4)$ & $2.87(4)$ & 0.1 & $8.61 \pm 1.0$ & $0.0247(0)$ & $2.645(4) \times 10^{37}$ \\
\hline 1238 & 58753.940308787 & $564.1(6)$ & $2.6(4)$ & 0.4 & $9.72 \pm 1.3$ & $0.0253(1)$ & $2.705(5) \times 10^{37}$ \\
\hline 1239 & 58753.940524758 & $568.9(6)$ & $15.73(9)$ & 0.1 & $5.97 \pm 0.6$ & $0.0939(1)$ & $1.005(1) \times 10^{38}$ \\
\hline 1240 & 58753.940798544 & $567.1(3)$ & $7.44(7)$ & 0.12 & $9.7 \pm 0.7$ & $0.0722(0)$ & $7.725(4) \times 10^{37}$ \\
\hline 1241 & 58753.941132301 & $562.9(2)$ & $4.81(3)$ & 0.3 & $20.63 \pm 0.9$ & $0.0992(0)$ & $1.062(0) \times 10^{38}$ \\
\hline 1242 & 58753.941361851 & $569.5(7)$ & $2.79(0)$ & 0.25 & $216.43 \pm 1.5$ & $0.6038(0)$ & $6.464(0) \times 10^{38}$ \\
\hline 1243 & 58753.942148752 & $567.7(8)$ & $2.21(1)$ & 0.21 & $54.91 \pm 1.2$ & $0.1213(0)$ & $1.299(0) \times 10^{38}$ \\
\hline 1244 & 58753.942148780 & $565.2(3)$ & $1.45(2)$ & 0.18 & $29.88 \pm 1.2$ & $0.0433(0)$ & $4.638(3) \times 10^{37}$ \\
\hline 1245 & 58753.942511094 & $568.0(5)$ & $1.84(0)$ & 0.17 & $133.38 \pm 1.4$ & $0.2454(0)$ & $2.627(0) \times 10^{38}$ \\
\hline 1246 & 58753.942549935 & $563.2(5)$ & $4.2(6)$ & 0.21 & $8.128 \pm 0.1$ & $0.0342(0)$ & $3.657(3) \times 10^{37}$ \\
\hline 1247 & 58753.945441245 & $568.0(8)$ & $2.1(3)$ & 0.08 & $10.31 \pm 1.1$ & $0.0216(0)$ & $2.318(3) \times 10^{37}$ \\
\hline 1248 & 58753.946301394 & $568.1(4)$ & $8.06(9)$ & 0.11 & $4.8 \pm 0.7$ & $0.0387(1)$ & $4.141(9) \times 10^{37}$ \\
\hline 1249 & 58753.946776275 & $567.9(8)$ & $4.74(3)$ & 0.4 & $15.71 \pm 0.8$ & $0.0745(0)$ & $7.971(2) \times 10^{37}$ \\
\hline 1250 & 58753.946776744 & $567.1(8)$ & $7.23(8)$ & 0.4 & $8.82 \pm 0.7$ & $0.0638(1)$ & $6.826(5) \times 10^{37}$ \\
\hline 1251 & 58753.946885822 & $565.1(3)$ & 7.47 ( 1 ) & 0.45 & $176.2 \pm 1.0$ & $1.3162(0)$ & $1.409(0) \times 10^{39}$ \\
\hline 1252 & 58753.947970664 & $567.3(5)$ & $4.27(4)$ & 0.12 & $12.38 \pm 0.8$ & $0.0529(0)$ & $5.659(3) \times 10^{37}$ \\
\hline 1253 & 58753.947970711 & $567.5(2)$ & $1.78(8)$ & 0.12 & $3.57 \pm 1.3$ & $0.0063(1)$ & $6.802(110) \times 10^{36}$ \\
\hline 1254 & 58753.949288479 & $567.6(7)$ & $2.28(3)$ & 0.04 & $10.55 \pm 1.0$ & $0.0240(0)$ & $2.575(2) \times 10^{37}$ \\
\hline
\end{tabular}


Table 1:

\begin{tabular}{|c|c|c|c|c|c|c|c|}
\hline Burst ID & $\begin{array}{c}\text { Burst time }^{a)} \\
\text { (MJD) }\end{array}$ & $\begin{array}{c}\mathrm{DM} \\
\left(\mathrm{pc} \mathrm{cm}^{-3}\right)\end{array}$ & $\begin{array}{l}\text { Width } \\
(\mathrm{ms})\end{array}$ & $\begin{array}{c}\text { Bandwidth }^{b)} \\
(\mathrm{GHz})\end{array}$ & $\begin{array}{c}\text { Peak Flux } \\
(\mathrm{mJy})\end{array}$ & $\begin{array}{c}\text { Fluence } \\
\text { (Jy ms) }\end{array}$ & $\begin{array}{c}\text { Energy } \\
\text { (erg) }\end{array}$ \\
\hline 1255 & 58753.952392833 & $565.1(4)$ & $7.42(6)$ & 0.12 & $9.47 \pm 0.7$ & $0.0703(0)$ & $7.522(4) \times 10^{37}$ \\
\hline 1256 & 58753.952772196 & $565.4(3)$ & $3.0(2)$ & 0.3 & $24.7 \pm 1.1$ & $0.0741(0)$ & $7.932(1) \times 10^{37}$ \\
\hline 1257 & 58753.952772217 & $566.3(4)$ & $3.51(2)$ & 0.12 & $11.87 \pm 0.2$ & $0.0417(0)$ & $4.460(0) \times 10^{37}$ \\
\hline 1258 & 58753.952825963 & $566.8(3)$ & $4.46(4)$ & 0.1 & $12.2 \pm 0.9$ & $0.0544(0)$ & $5.825(4) \times 10^{37}$ \\
\hline 1259 & 58753.952826043 & $566.9(4)$ & $3.3(3)$ & 0.12 & $12.86 \pm 1.1$ & $0.0424(0)$ & $4.543(3) \times 10^{37}$ \\
\hline 1260 & 58753.952903531 & $563.2(4)$ & $2.66(8)$ & 0.12 & $5.56 \pm 1.4$ & $0.0148(1)$ & $1.583(12) \times 10^{37}$ \\
\hline 1262 & 58754.984296416 & $566.0(5)$ & $3.49(5)$ & 0.16 & $27.188 \pm 0.3$ & $0.0949(1)$ & $1.016(1) \times 10^{38}$ \\
\hline 1263 & 58754.984941175 & $565.6(5)$ & $2.99(5)$ & 0.1 & $22.41 \pm 1.6$ & $0.0670(1)$ & $7.173(7) \times 10^{37}$ \\
\hline 1264 & 58754.984941212 & $565.6(5)$ & $2.7(4)$ & 0.4 & $23.95 \pm 1.9$ & $0.0647(1)$ & $6.922(7) \times 10^{37}$ \\
\hline 1265 & 58754.984941335 & $565.6(5)$ & $5.11(6)$ & 0.36 & $12.01 \pm 1.1$ & $0.0614(1)$ & $6.570(6) \times 10^{37}$ \\
\hline 1266 & 58754.987095746 & $569.2(7)$ & $3.92(7)$ & 0.4 & $8.49 \pm 1.2$ & $0.0333(1)$ & $3.563(8) \times 10^{37}$ \\
\hline 1267 & 58754.987208332 & $565.1(4)$ & $2.89(9)$ & 0.3 & $7.83 \pm 2.0$ & $0.0226(2)$ & $2.422(19) \times 10^{37}$ \\
\hline 1268 & 58754.987208380 & $565.1(4)$ & $4.47(9)$ & 0.3 & $11.42 \pm 1.2$ & $0.0510(1)$ & $5.465(12) \times 10^{37}$ \\
\hline 1269 & 58754.987209118 & $566.5(7)$ & $5.66(7)$ & 0.2 & $10.71 \pm 1.1$ & $0.0606(1)$ & $6.489(7) \times 10^{37}$ \\
\hline 1271 & 58754.987719067 & $565.6(4)$ & $3.04(3)$ & 0.03 & $18.37 \pm 1.4$ & $0.0558(0)$ & $5.978(3) \times 10^{37}$ \\
\hline 1272 & 58754.987733048 & $567.1(3)$ & $3.41(4)$ & 0.03 & $14.22 \pm 1.3$ & $0.0485(1)$ & $5.191(5) \times 10^{37}$ \\
\hline 1273 & 58754.989178262 & $568.0(10)$ & $7.63(8)$ & 0.1 & $10.91 \pm 0.9$ & $0.0832(1)$ & $8.911(7) \times 10^{37}$ \\
\hline 1274 & 58754.989552169 & $566.0(8)$ & $3.98(9)$ & 0.2 & $7.16 \pm 8.0$ & $0.0285(19)$ & $3.051(206) \times 10^{37}$ \\
\hline 1275 & 58754.989552214 & $567.1(6)$ & $7.75(9)$ & 0.2 & $7.1 \pm 3.3$ & $0.0550(14)$ & $5.890(153) \times 10^{37}$ \\
\hline 1276 & 58754.990572445 & $566.7(6)$ & $3.59(4)$ & 0.06 & $14.38 \pm 1.2$ & $0.0516(0)$ & $5.526(4) \times 10^{37}$ \\
\hline 1277 & 58754.990710551 & $568.1(6)$ & $3.82(5)$ & 0.15 & $12.89 \pm 1.3$ & $0.0492(1)$ & $5.271(6) \times 10^{37}$ \\
\hline 1278 & 58754.991775229 & $567.9(7)$ & $3.79(7)$ & 0.12 & $7.95 \pm 1.1$ & $0.0301(1)$ & $3.225(8) \times 10^{37}$ \\
\hline 1279 & 58754.992775800 & $564.2(5)$ & $1.34(3)$ & 0.14 & $22.19 \pm 1.2$ & $0.0297(0)$ & $3.183(4) \times 10^{37}$ \\
\hline 1280 & 58754.992775813 & $567.1(4)$ & $2.43(2)$ & 0.4 & $24.0 \pm 1.4$ & $0.0583(0)$ & $6.243(2) \times 10^{37}$ \\
\hline 1281 & 58754.993681280 & $566.6(4)$ & $3.63(4)$ & 0.37 & $14.95 \pm 1.3$ & $0.0543(1)$ & $5.809(5) \times 10^{37}$ \\
\hline 1282 & 58754.993681362 & $565.9(5)$ & $6.07(4)$ & 0.31 & $20.46 \pm 1.1$ & $0.1242(0)$ & $1.329(0) \times 10^{38}$ \\
\hline 1283 & 58754.994496278 & $566.5(3)$ & $1.57(3)$ & 0.36 & $12.69 \pm 1.9$ & $0.0199(1)$ & $2.133(5) \times 10^{37}$ \\
\hline 1284 & 58754.995154626 & $564.9(3)$ & $3.13(4)$ & 0.13 & $13.5 \pm 1.4$ & $0.0423(1)$ & $4.523(5) \times 10^{37}$ \\
\hline 1285 & 58754.996040469 & $568.9(7)$ & $2.37(4)$ & 0.02 & $9.96 \pm 1.5$ & $0.0236(1)$ & $2.527(7) \times 10^{37}$ \\
\hline 1286 & 58754.996043103 & $567.9(6)$ & $7.02(9)$ & 0.069 & $7.46 \pm 0.9$ & $0.0524(1)$ & $5.606(10) \times 10^{37}$ \\
\hline 1289 & 58755.000407219 & $564.4(3)$ & $2.0(0)$ & 0.32 & $210.78 \pm 1.9$ & $0.4216(0)$ & $4.513(0) \times 10^{38}$ \\
\hline 1290 & 58755.000407238 & $566.4(12)$ & $1.8(1)$ & 0.35 & $213.84 \pm 1.5$ & $0.3849(0)$ & $4.120(0) \times 10^{38}$ \\
\hline 1291 & 58755.000445434 & $565.7(5)$ & $4.82(6)$ & 0.14 & $8.52 \pm 0.9$ & $0.0411(1)$ & $4.396(6) \times 10^{37}$ \\
\hline 1292 & 58755.000445904 & $565.7(5)$ & $4.26(6)$ & 0.13 & $9.58 \pm 1.1$ & $0.0408(1)$ & $4.369(6) \times 10^{37}$ \\
\hline 1293 & 58755.000548752 & $566.7(2)$ & $4.74(4)$ & 0.12 & $13.95 \pm 0.9$ & $0.0661(0)$ & $7.078(3) \times 10^{37}$ \\
\hline 1294 & 58755.000548763 & $565.4(4)$ & $4.52(5)$ & 0.1 & $12.12 \pm 0.7$ & $0.0548(0)$ & $5.864(3) \times 10^{37}$ \\
\hline 1295 & 58755.003864649 & $568.1(5)$ & $4.74(7)$ & 0.11 & $4.552 \pm 0.1$ & $0.0216(0)$ & $2.311(2) \times 10^{37}$ \\
\hline 1296 & 58755.004740650 & $561.9(5)$ & $6.03(9)$ & 0.2 & $4.651 \pm 0.1$ & $0.0280(0)$ & $3.000(3) \times 10^{37}$ \\
\hline 1297 & 58755.006241824 & $564.7(4)$ & $12.95(9)$ & 0.2 & $8.57 \pm 0.8$ & $0.1110(1)$ & $1.188(1) \times 10^{38}$ \\
\hline 1298 & 58755.006592410 & $565.7(3)$ & $3.45(5)$ & 0.1 & $10.29 \pm 1.3$ & $0.0355(1)$ & $3.800(7) \times 10^{37}$ \\
\hline 1299 & 58755.006875758 & $565.2(3)$ & $6.22(9)$ & 0.045 & $6.63 \pm 0.8$ & $0.0412(1)$ & $4.415(7) \times 10^{37}$ \\
\hline 1300 & 58755.007868091 & $565.2(3)$ & $3.38(3)$ & 0.1 & $15.12 \pm 1.1$ & $0.0511(0)$ & $5.471(3) \times 10^{37}$ \\
\hline 1301 & 58755.008651718 & $568.7(5)$ & $6.57(9)$ & 0.2 & $6.28 \pm 0.8$ & $0.0413(1)$ & $4.417(8) \times 10^{37}$ \\
\hline 1302 & 58755.013064749 & $563.7(3)$ & 4.09 ( 1 ) & 0.24 & $72.75 \pm 1.2$ & $0.2975(0)$ & $3.185(0) \times 10^{38}$ \\
\hline 1303 & 58755.013617755 & $566.0(4)$ & $3.66(4)$ & 0.13 & $22.19 \pm 1.1$ & $0.0812(0)$ & $8.694(4) \times 10^{37}$ \\
\hline 1304 & 58755.013617786 & $567.4(8)$ & $1.43(5)$ & 0.13 & $7.76 \pm 2.6$ & $0.0111(1)$ & $1.188(14) \times 10^{37}$ \\
\hline 1305 & 58755.013887775 & $568.1(3)$ & $2.3(3)$ & 0.12 & $15.25 \pm 1.4$ & $0.0351(0)$ & $3.755(3) \times 10^{37}$ \\
\hline 1306 & 58755.013940489 & $568.5(5)$ & $4.39(6)$ & 0.3 & $5.118 \pm 0.1$ & $0.0225(0)$ & $2.404(3) \times 10^{37}$ \\
\hline 1307 & 58755.014108161 & $567.2(3)$ & $4.61(6)$ & 0.04 & $9.66 \pm 1.1$ & $0.0445(1)$ & $4.767(6) \times 10^{37}$ \\
\hline 1308 & 58755.014420926 & $564.3(3)$ & $5.69(7)$ & 0.04 & $10.5 \pm 1.0$ & $0.0597(1)$ & $6.396(7) \times 10^{37}$ \\
\hline 1309 & 58755.014421395 & $566.3(5)$ & $4.05(6)$ & 0.1 & $9.33 \pm 1.2$ & $0.0378(1)$ & $4.045(7) \times 10^{37}$ \\
\hline 1310 & 58755.016778532 & $566.9(4)$ & $4.72(5)$ & 0.09 & $14.52 \pm 1.2$ & $0.0685(1)$ & $7.336(6) \times 10^{37}$ \\
\hline 1311 & 58755.017582020 & $566.2(6)$ & $3.51(4)$ & 0.3 & $14.26 \pm 1.5$ & $0.0500(1)$ & $5.358(7) \times 10^{37}$ \\
\hline 1312 & 58755.018422613 & $564.7(2)$ & $2.64(3)$ & 0.19 & $16.24 \pm 1.6$ & $0.0429(1)$ & $4.590(5) \times 10^{37}$ \\
\hline 1313 & 58755.020525183 & $569.2(7)$ & $5.34(5)$ & 0.2 & $16.89 \pm 1.2$ & $0.0902(1)$ & $9.655(6) \times 10^{37}$ \\
\hline 1314 & 58755.021036502 & $563.3(3)$ & $6.99(6)$ & 0.18 & $15.54 \pm 1.1$ & $0.1086(1)$ & $1.163(0) \times 10^{38}$ \\
\hline 1315 & 58755.021082202 & $563.3(3)$ & $6.13(4)$ & 0.21 & $26.68 \pm 1.3$ & $0.1636(0)$ & $1.751(0) \times 10^{38}$ \\
\hline 1316 & 58755.021676912 & $570.0(5)$ & $3.94(6)$ & 0.1 & $12.39 \pm 1.5$ & $0.0488(1)$ & $5.226(9) \times 10^{37}$ \\
\hline 1317 & 58755.021859377 & $567.6(6)$ & $4.53(7)$ & 0.12 & $11.35 \pm 1.4$ & $0.0514(1)$ & $5.504(10) \times 10^{37}$ \\
\hline
\end{tabular}


Table 1:

\begin{tabular}{|c|c|c|c|c|c|c|c|}
\hline Burst ID & $\begin{array}{c}\text { Burst time }^{a)} \\
\text { (MJD) }\end{array}$ & 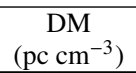 & $\begin{array}{l}\text { Width } \\
\text { (ms) }\end{array}$ & $\begin{array}{c}\left.\text { Bandwidth }^{b}\right) \\
(\mathrm{GHz})\end{array}$ & $\begin{array}{c}\begin{array}{c}\text { Peak Flux } \\
(\mathrm{mJy})\end{array} \\
\end{array}$ & $\begin{array}{c}\text { Fluence } \\
\text { (Jy ms) }\end{array}$ & $\begin{array}{c}\text { Energy } \\
\text { (erg) }\end{array}$ \\
\hline 1318 & 58755.022140157 & $566.2(5)$ & $3.55(3)$ & 0.15 & $20.77 \pm 1.5$ & $0.0737(0)$ & $7.893(4) \times 10^{37}$ \\
\hline 1319 & 58755.022909893 & $564.4(6)$ & $3.15(4)$ & 0.115 & $16.11 \pm 1.8$ & $0.0508(1)$ & $5.432(7) \times 10^{37}$ \\
\hline 1320 & 58755.023399422 & $566.0(5)$ & $3.63(5)$ & 0.24 & $20.057 \pm 0.3$ & $0.0728(1)$ & $7.798(12) \times 10^{37}$ \\
\hline 1321 & 58755.023566549 & $568.1(5)$ & $4.64(7)$ & 0.31 & $16.713 \pm 0.2$ & $0.0775(1)$ & $8.294(13) \times 10^{37}$ \\
\hline 1322 & 58755.885045688 & $566.0(5)$ & $5.58(8)$ & 0.24 & $13.476 \pm 0.1$ & $0.0751(1)$ & $8.044(7) \times 10^{37}$ \\
\hline 1323 & 58755.885112829 & $565.9(5)$ & $5.23(8)$ & 0.18 & $11.747 \pm 0.1$ & $0.0615(1)$ & $6.581(6) \times 10^{37}$ \\
\hline 1325 & 58755.887770633 & $567.2(5)$ & $6.16(9)$ & 0.065 & $5.663 \pm 0.1$ & $0.0349(0)$ & $3.734(3) \times 10^{37}$ \\
\hline 1326 & 58755.887916349 & $566.0(5)$ & $3.1(5)$ & 0.07 & $14.416 \pm 0.1$ & $0.0446(0)$ & $4.779(4) \times 10^{37}$ \\
\hline 1327 & 58755.889345460 & $566.0(5)$ & $3.86(6)$ & 0.31 & $13.534 \pm 0.1$ & $0.0523(0)$ & $5.597(5) \times 10^{37}$ \\
\hline 1328 & 58755.889551295 & $566.0(5)$ & $5.31(8)$ & 0.2 & $18.303 \pm 0.1$ & $0.0973(1)$ & $1.041(0) \times 10^{38}$ \\
\hline 1329 & 58755.892229271 & $566.0(5)$ & $3.68(5)$ & 0.45 & $218.013 \pm 1.8$ & $0.8025(7)$ & $8.591(7) \times 10^{38}$ \\
\hline 1330 & 58755.892238170 & $565.7(0)$ & $2.77(4)$ & 0.3 & $9.78 \pm 1.1$ & $0.0271(0)$ & $2.900(3) \times 10^{37}$ \\
\hline 1331 & 58755.892241124 & $567.7(5)$ & $1.62(3)$ & 0.2 & $8.24 \pm 1.4$ & $0.0134(0)$ & $1.429(4) \times 10^{37}$ \\
\hline 1332 & 58755.893744435 & $565.9(5)$ & $8.19(9)$ & 0.15 & $9.358 \pm 0.1$ & $0.0766(1)$ & $8.204(7) \times 10^{37}$ \\
\hline 1334 & 58755.894632086 & $566.0(5)$ & $4.67(7)$ & 0.19 & $20.058 \pm 0.2$ & $0.0936(1)$ & $1.002(0) \times 10^{38}$ \\
\hline 1335 & 58755.895460954 & $564.9(5)$ & $3.45(5)$ & 0.3 & $8.05 \pm 0.9$ & $0.0278(0)$ & $2.973(4) \times 10^{37}$ \\
\hline 1336 & 58755.895540443 & $566.0(5)$ & $3.57(5)$ & 0.29 & $24.037 \pm 0.2$ & $0.0859(1)$ & $9.190(8) \times 10^{37}$ \\
\hline 1337 & 58755.895963795 & $567.6(5)$ & $8.49(9)$ & 0.18 & $8.641 \pm 0.1$ & $0.0733(1)$ & $7.850(7) \times 10^{37}$ \\
\hline 1338 & 58755.898783886 & $566.0(5)$ & $3.11(5)$ & 0.27 & $211.127 \pm 1.6$ & $0.6567(6)$ & $7.030(6) \times 10^{38}$ \\
\hline 1339 & 58755.898816111 & $568.5(5)$ & $8.32(9)$ & 0.1 & $8.674 \pm 0.1$ & $0.0722(1)$ & $7.727(6) \times 10^{37}$ \\
\hline 1340 & 58755.899096592 & $562.8(5)$ & $8.33(9)$ & 0.19 & $5.003 \pm 0.0$ & $0.0417(0)$ & $4.461(3) \times 10^{37}$ \\
\hline 1341 & 58755.899138835 & $564.5(5)$ & $3.98(6)$ & 0.09 & $3.867 \pm 0.0$ & $0.0154(0)$ & $1.647(1) \times 10^{37}$ \\
\hline 1342 & 58756.843182037 & $566.0(5)$ & $2.6(4)$ & 0.22 & $25.396 \pm 0.3$ & $0.0660(1)$ & $7.064(9) \times 10^{37}$ \\
\hline 1343 & 58756.843306344 & $566.0(5)$ & $3.83(6)$ & 0.17 & $12.595 \pm 0.1$ & $0.0482(1)$ & $5.158(6) \times 10^{37}$ \\
\hline 1344 & 58756.843764668 & $566.0(5)$ & $4.12(6)$ & 0.3 & $35.519 \pm 0.4$ & $0.1464(2)$ & $1.567(2) \times 10^{38}$ \\
\hline 1345 & 58756.845079396 & $569.8(5)$ & $6.45(9)$ & 0.21 & $4.175 \pm 0.1$ & $0.0269(0)$ & $2.885(3) \times 10^{37}$ \\
\hline 1346 & 58756.846970180 & $567.3(4)$ & $2.38(2)$ & 0.22 & $24.39 \pm 1.6$ & $0.0580(0)$ & $6.214(3) \times 10^{37}$ \\
\hline 1347 & 58756.846971140 & $566.8(3)$ & $4.37(7)$ & 0.2 & $8.76 \pm 1.2$ & $0.0383(1)$ & $4.098(8) \times 10^{37}$ \\
\hline 1348 & 58756.847036296 & $566.7(3)$ & $3.4(4)$ & 0.09 & $13.43 \pm 1.3$ & $0.0457(0)$ & $4.888(5) \times 10^{37}$ \\
\hline 1349 & 58756.847394000 & $566.2(6)$ & $2.92(2)$ & 0.27 & $24.67 \pm 1.4$ & $0.0720(0)$ & $7.711(3) \times 10^{37}$ \\
\hline 1352 & 58756.849107671 & $565.5(6)$ & $3.0(3)$ & 0.4 & $16.75 \pm 1.5$ & $0.0503(0)$ & $5.379(4) \times 10^{37}$ \\
\hline 1353 & 58756.849107875 & $568.6(7)$ & $2.26(8)$ & 0.45 & $7.03 \pm 2.1$ & $0.0159(2)$ & $1.701(18) \times 10^{37}$ \\
\hline 1354 & 58756.849736535 & $566.0(4)$ & $3.66(5)$ & 0.1 & $10.48 \pm 1.2$ & $0.0384(1)$ & $4.106(5) \times 10^{37}$ \\
\hline 1355 & 58756.850108749 & $566.4(2)$ & $3.68(4)$ & 0.45 & $15.35 \pm 1.2$ & $0.0565(0)$ & $6.047(4) \times 10^{37}$ \\
\hline 1356 & 58756.850223938 & $566.4(2)$ & $3.61(5)$ & 0.2 & $13.02 \pm 1.4$ & $0.0470(1)$ & $5.032(6) \times 10^{37}$ \\
\hline 1357 & 58756.850224192 & $565.7(6)$ & $3.91(3)$ & 0.2 & $19.05 \pm 1.3$ & $0.0745(0)$ & $7.974(4) \times 10^{37}$ \\
\hline 1358 & 58756.850858274 & $565.9(3)$ & $2.41(1)$ & 0.45 & $241.91 \pm 8.6$ & $0.5830(0)$ & $6.241(0) \times 10^{38}$ \\
\hline 1359 & 58756.850858300 & $565.9(3)$ & $2.78(0)$ & 0.45 & $394.88 \pm 5.8$ & $1.0978(0)$ & $1.175(0) \times 10^{39}$ \\
\hline 1360 & 58756.851647064 & $565.8(4)$ & $4.58(3)$ & 0.32 & $18.94 \pm 1.1$ & $0.0867(0)$ & $9.286(3) \times 10^{37}$ \\
\hline 1361 & 58756.851647120 & $567.2(4)$ & $3.45(2)$ & 0.21 & $16.21 \pm 1.3$ & $0.0559(0)$ & $5.987(3) \times 10^{37}$ \\
\hline 1362 & 58756.851651577 & $566.7(3)$ & $3.79(3)$ & 0.3 & $17.3 \pm 1.2$ & $0.0656(0)$ & $7.019(3) \times 10^{37}$ \\
\hline 1363 & 58756.851651620 & $569.0(5)$ & $3.21(5)$ & 0.26 & $17.7 \pm 1.0$ & $0.0568(0)$ & $6.082(4) \times 10^{37}$ \\
\hline 1364 & 58756.851702464 & $565.8(3)$ & $5.7(2)$ & 0.2 & $75.0 \pm 1.1$ & $0.4275(0)$ & $4.576(0) \times 10^{38}$ \\
\hline 1365 & 58756.851702537 & $565.4(5)$ & $5.01(6)$ & 0.2 & $18.88 \pm 1.2$ & $0.0946(1)$ & $1.013(0) \times 10^{38}$ \\
\hline 1366 & 58756.852097829 & $566.4(5)$ & $2.36(2)$ & 0.06 & $25.13 \pm 1.6$ & $0.0593(0)$ & $6.349(2) \times 10^{37}$ \\
\hline 1367 & 58756.852097863 & $565.3(5)$ & $2.1(6)$ & 0.15 & $25.85 \pm 1.2$ & $0.0543(1)$ & $5.811(7) \times 10^{37}$ \\
\hline 1368 & 58756.852515302 & $567.4(3)$ & $2.05(2)$ & 0.08 & $25.47 \pm 1.7$ & $0.0522(0)$ & $5.589(2) \times 10^{37}$ \\
\hline 1369 & 58756.852515311 & $566.5(3)$ & $2.03(3)$ & 0.12 & $27.41 \pm 1.5$ & $0.0556(0)$ & $5.956(4) \times 10^{37}$ \\
\hline 1370 & 58756.852784688 & $566.2(5)$ & $7.03(7)$ & 0.08 & $10.68 \pm 0.9$ & $0.0751(1)$ & $8.037(7) \times 10^{37}$ \\
\hline 1371 & 58756.853475520 & $567.8(5)$ & $2.73(6)$ & 0.2 & $16.33 \pm 5.0$ & $0.0446(3)$ & $4.772(31) \times 10^{37}$ \\
\hline 1372 & 58756.853475558 & $569.1(7)$ & $6.02(9)$ & 0.2 & $5.35 \pm 1.6$ & $0.0322(6)$ & $3.448(61) \times 10^{37}$ \\
\hline 1373 & 58756.855021790 & $565.8(4)$ & $4.27(2)$ & 0.13 & $27.4 \pm 1.3$ & $0.1170(0)$ & $1.252(0) \times 10^{38}$ \\
\hline 1374 & 58756.855021841 & $565.8(4)$ & $3.4(4)$ & 0.11 & $30.1 \pm 1.1$ & $0.1023(0)$ & $1.096(0) \times 10^{38}$ \\
\hline 1375 & 58756.855022586 & $565.4(5)$ & $3.45(5)$ & 0.3 & $10.81 \pm 1.2$ & $0.0373(1)$ & $3.992(6) \times 10^{37}$ \\
\hline 1376 & 58756.855152253 & $566.0(4)$ & $4.9(7)$ & 0.15 & $12.39 \pm 1.8$ & 0.0607 ( 1) & $6.499(13) \times 10^{37}$ \\
\hline 1377 & 58756.856002153 & $565.3(4)$ & $1.94(1)$ & 0.17 & $31.46 \pm 1.6$ & $0.0610(0)$ & $6.533(2) \times 10^{37}$ \\
\hline 1378 & 58756.856005325 & $566.9(8)$ & $4.81(6)$ & 0.2 & $10.32 \pm 1.1$ & $0.0496(1)$ & $5.314(6) \times 10^{37}$ \\
\hline 1379 & 58756.857136633 & $565.2(6)$ & $2.79(3)$ & 0.4 & $17.27 \pm 1.4$ & $0.0482(0)$ & $5.158(3) \times 10^{37}$ \\
\hline 1380 & 58756.857136946 & $566.9(4)$ & $6.77(7)$ & 0.4 & $10.37 \pm 0.8$ & $0.0702(1)$ & $7.515(6) \times 10^{37}$ \\
\hline
\end{tabular}


Table 1:

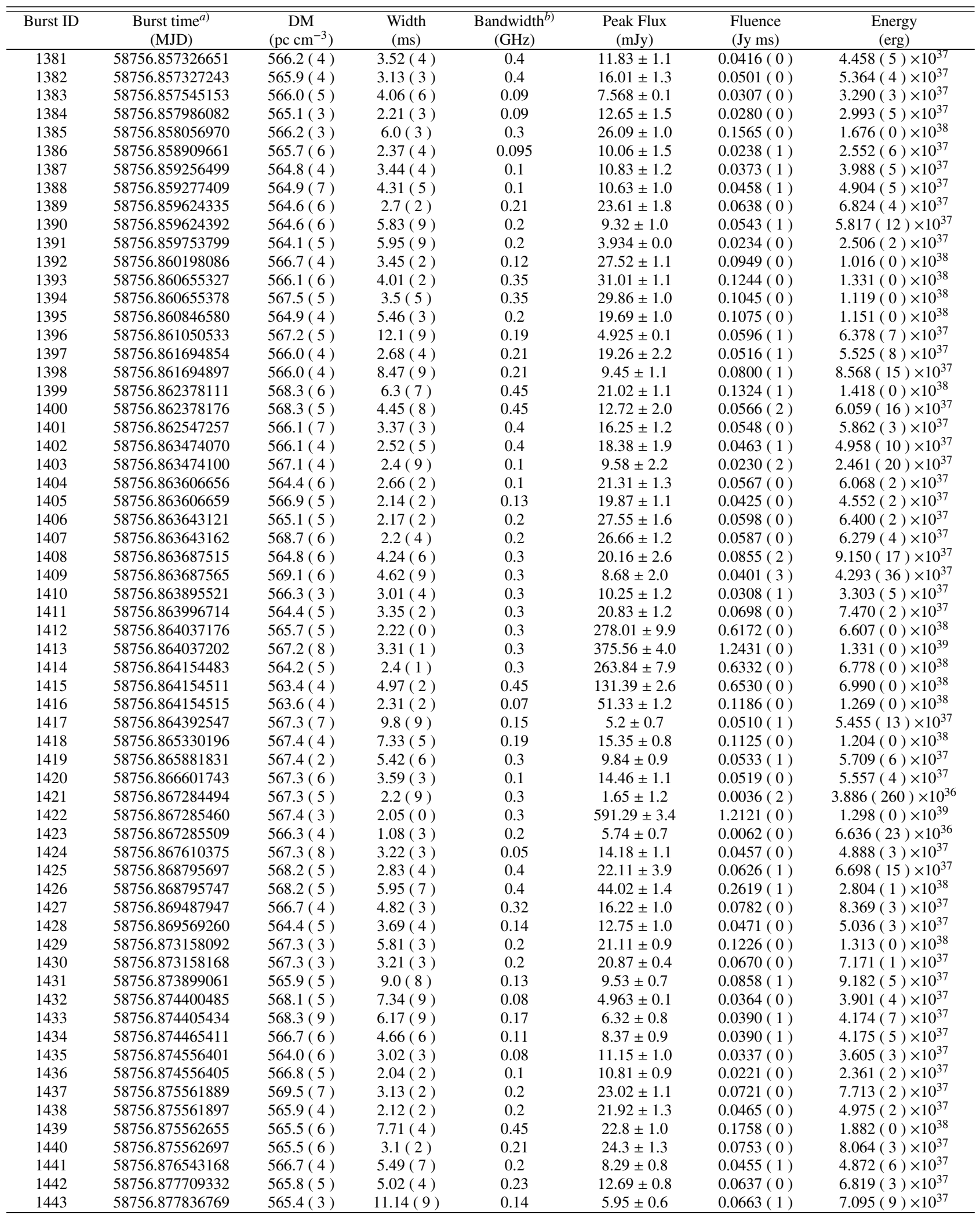


Table 1:

\begin{tabular}{|c|c|c|c|c|c|c|c|}
\hline Burst ID & $\begin{array}{c}\text { Burst time }^{a)} \\
\text { (MJD) }\end{array}$ & $\begin{array}{c}\mathrm{DM} \\
(\mathrm{pc} \mathrm{cm}-3)\end{array}$ & $\begin{array}{l}\text { Width } \\
\text { (ms) }\end{array}$ & $\begin{array}{c}\left.\text { Bandwidth }^{b}\right) \\
(\mathrm{GHz})\end{array}$ & $\begin{array}{c}\begin{array}{c}\text { Peak Flux } \\
(\mathrm{mJy})\end{array} \\
\end{array}$ & $\begin{array}{c}\text { Fluence } \\
\text { (Jy ms) }\end{array}$ & $\begin{array}{c}\text { Energy } \\
\text { (erg) }\end{array}$ \\
\hline 1444 & 58756.878005118 & $566.6(4)$ & $4.93(5)$ & 0.45 & $11.6 \pm 0.9$ & $0.0572(0)$ & $6.122(4) \times 10^{37}$ \\
\hline 1445 & 58756.878005229 & $564.2(6)$ & $7.57(9)$ & 0.4 & $7.45 \pm 0.8$ & $0.0564(1)$ & $6.037(7) \times 10^{37}$ \\
\hline 1446 & 58756.878005259 & $566.6(4)$ & $3.21(5)$ & 0.1 & $6.32 \pm 0.8$ & $0.0203(0)$ & $2.172(3) \times 10^{37}$ \\
\hline 1447 & 58756.878568783 & $566.2(3)$ & $3.44(4)$ & 0.05 & $10.98 \pm 1.0$ & $0.0378(0)$ & $4.043(3) \times 10^{37}$ \\
\hline 1448 & 58756.878568788 & $566.5(3)$ & $2.76(6)$ & 0.07 & $11.23 \pm 0.9$ & $0.0310(0)$ & $3.318(5) \times 10^{37}$ \\
\hline 1449 & 58756.878747573 & $566.7(5)$ & $5.31(8)$ & 0.07 & $7.179 \pm 0.1$ & $0.0381(0)$ & $4.078(4) \times 10^{37}$ \\
\hline 1451 & 58756.880155134 & $569.4(9)$ & $4.39(4)$ & 0.1 & $11.17 \pm 0.9$ & $0.0490(0)$ & $5.249(4) \times 10^{37}$ \\
\hline 1452 & 58756.880155148 & $564.2(5)$ & $3.21(5)$ & 0.1 & $10.92 \pm 0.9$ & $0.0350(0)$ & $3.752(5) \times 10^{37}$ \\
\hline 1453 & 58756.880156867 & $566.0(4)$ & $6.48(8)$ & 0.11 & $8.03 \pm 0.8$ & $0.0520(1)$ & $5.570(6) \times 10^{37}$ \\
\hline 1454 & 58756.880952123 & $566.4(6)$ & $3.33(3)$ & 0.04 & $14.68 \pm 1.1$ & $0.0489(0)$ & $5.233(3) \times 10^{37}$ \\
\hline 1455 & 58756.881128916 & $566.2(5)$ & $2.03(1)$ & 0.15 & $76.93 \pm 1.5$ & $0.1562(0)$ & $1.672(0) \times 10^{38}$ \\
\hline 1456 & 58756.881128926 & $566.5(4)$ & $3.1(3)$ & 0.16 & $75.39 \pm 1.6$ & $0.2337(0)$ & $2.502(0) \times 10^{38}$ \\
\hline 1457 & 58756.881851668 & $566.2(4)$ & $4.11(3)$ & 0.18 & $15.07 \pm 1.0$ & $0.0619(0)$ & $6.630(3) \times 10^{37}$ \\
\hline 1458 & 58756.881851695 & $566.4(4)$ & $4.56(6)$ & 0.12 & $14.19 \pm 0.9$ & $0.0647(0)$ & $6.927(5) \times 10^{37}$ \\
\hline 1460 & 58757.900647731 & $565.5(2)$ & $3.43(5)$ & 0.2 & $8.7 \pm 1.0$ & $0.0298(0)$ & $3.194(4) \times 10^{37}$ \\
\hline 1461 & 58757.900647870 & $565.5(2)$ & $3.39(2)$ & 0.3 & $25.82 \pm 1.0$ & $0.0875(0)$ & $9.370(1) \times 10^{37}$ \\
\hline 1462 & 58757.900652212 & $566.5(5)$ & $1.89(2)$ & 0.27 & $12.54 \pm 1.3$ & $0.0237(0)$ & $2.537(2) \times 10^{37}$ \\
\hline 1463 & 58757.900851357 & $568.9(8)$ & $6.6(7)$ & 0.3 & $7.46 \pm 0.7$ & $0.0492(0)$ & $5.271(5) \times 10^{37}$ \\
\hline 1464 & 58757.900851868 & $567.1(6)$ & $4.11(8)$ & 0.25 & $5.08 \pm 0.8$ & $0.0209(1)$ & $2.235(7) \times 10^{37}$ \\
\hline 1465 & 58757.901426407 & $567.0(12)$ & $6.34(9)$ & 0.09 & $4.53 \pm 0.8$ & $0.0287(1)$ & $3.074(11) \times 10^{37}$ \\
\hline 1466 & 58757.902634777 & $566.6(5)$ & $4.31(2)$ & 0.45 & $56.57 \pm 2.7$ & $0.2438(0)$ & $2.610(0) \times 10^{38}$ \\
\hline 1467 & 58757.902634844 & $563.8(5)$ & $11.28(7)$ & 0.45 & $32.67 \pm 1.0$ & $0.3685(1)$ & $3.945(0) \times 10^{38}$ \\
\hline 1468 & 58757.903874114 & $566.1(4)$ & $2.76(1)$ & 0.18 & $26.29 \pm 1.1$ & $0.0726(0)$ & $7.767(1) \times 10^{37}$ \\
\hline 1469 & 58757.904270860 & $566.1(4)$ & $3.74(3)$ & 0.18 & $11.82 \pm 0.9$ & $0.0442(0)$ & $4.732(3) \times 10^{37}$ \\
\hline 1470 & 58757.906360825 & $565.8(3)$ & $2.33(2)$ & 0.15 & $16.11 \pm 1.1$ & $0.0375(0)$ & $4.018(2) \times 10^{37}$ \\
\hline 1471 & 58757.910407693 & $565.2(6)$ & $3.3(0)$ & 0.45 & $109.86 \pm 1.0$ & $0.3625(0)$ & $3.881(0) \times 10^{38}$ \\
\hline 1472 & 58757.910407763 & $569.0(7)$ & $2.8(4)$ & 0.05 & $5.12 \pm 0.8$ & $0.0143(0)$ & $1.535(3) \times 10^{37}$ \\
\hline 1473 & 58757.910442512 & $567.4(4)$ & $1.68(0)$ & 0.32 & $234.27 \pm 1.4$ & $0.3936(0)$ & $4.213(0) \times 10^{38}$ \\
\hline 1474 & 58757.910442566 & $564.2(3)$ & $1.43(2)$ & 0.32 & $231.91 \pm 1.2$ & $0.3316(0)$ & $3.550(0) \times 10^{38}$ \\
\hline 1475 & 58757.910996034 & $566.6(4)$ & $3.94(5)$ & 0.12 & $9.05 \pm 0.9$ & $0.0357(0)$ & $3.817(4) \times 10^{37}$ \\
\hline 1478 & 58757.911641774 & $567.8(7)$ & $6.88(9)$ & 0.12 & $6.12 \pm 0.7$ & $0.0421(1)$ & $4.507(6) \times 10^{37}$ \\
\hline 1479 & 58757.911642070 & $566.2(5)$ & $3.95(9)$ & 0.12 & $4.99 \pm 1.4$ & $0.0197(2)$ & $2.110(17) \times 10^{37}$ \\
\hline 1480 & 58757.912605398 & $561.5(5)$ & $8.94(9)$ & 0.11 & $5.663 \pm 0.0$ & $0.0506(0)$ & $5.420(4) \times 10^{37}$ \\
\hline 1481 & 58757.912752257 & $565.6(3)$ & $6.83(8)$ & 0.12 & $7.53 \pm 0.7$ & $0.0514(1)$ & $5.505(5) \times 10^{37}$ \\
\hline 1482 & 58757.912756800 & $566.0(4)$ & $2.51(2)$ & 0.12 & $18.02 \pm 1.1$ & $0.0452(0)$ & $4.842(1) \times 10^{37}$ \\
\hline 1483 & 58757.912757062 & $567.0(4)$ & $1.99(4)$ & 0.12 & $7.78 \pm 1.2$ & $0.0155(0)$ & $1.657(4) \times 10^{37}$ \\
\hline 1484 & 58757.914598243 & $564.4(3)$ & $3.98(4)$ & 0.12 & $10.63 \pm 0.9$ & $0.0423(0)$ & $4.529(4) \times 10^{37}$ \\
\hline 1485 & 58757.914789718 & $564.9(2)$ & $5.52(5)$ & 0.12 & $11.12 \pm 0.8$ & $0.0614(0)$ & $6.571(3) \times 10^{37}$ \\
\hline 1486 & 58757.914789776 & $567.5(5)$ & $4.33(6)$ & 0.12 & $4.3 \pm 0.9$ & $0.0186(1)$ & $1.993(5) \times 10^{37}$ \\
\hline 1487 & 58757.915948383 & $561.2(3)$ & $3.98(4)$ & 0.08 & $9.24 \pm 0.8$ & $0.0368(0)$ & $3.937(3) \times 10^{37}$ \\
\hline 1488 & 58757.916997218 & $567.9(7)$ & $5.47(6)$ & 0.08 & $8.07 \pm 0.7$ & $0.0441(0)$ & $4.725(4) \times 10^{37}$ \\
\hline 1489 & 58757.918666984 & $565.3(3)$ & $4.34(7)$ & 0.08 & $5.93 \pm 0.8$ & $0.0257(1)$ & $2.755(5) \times 10^{37}$ \\
\hline 1490 & 58757.919171542 & $565.5(6)$ & $19.75(9)$ & 0.08 & $4.88 \pm 0.7$ & $0.0964(3)$ & $1.032(3) \times 10^{38}$ \\
\hline 1491 & 58757.919244107 & $566.9(4)$ & $5.95(6)$ & 0.08 & $7.34 \pm 0.6$ & $0.0437(0)$ & $4.675(3) \times 10^{37}$ \\
\hline 1492 & 58757.919796998 & $564.6(2)$ & $4.13(1)$ & 0.4 & $33.47 \pm 0.9$ & $0.1382(0)$ & $1.480(0) \times 10^{38}$ \\
\hline 1493 & 58757.919797049 & $564.6(2)$ & $3.2(2)$ & 0.25 & $31.89 \pm 0.8$ & $0.1021(0)$ & $1.092(0) \times 10^{38}$ \\
\hline 1494 & 58757.920250584 & $565.3(3)$ & $3.11(2)$ & 0.22 & $23.01 \pm 1.0$ & $0.0716(0)$ & $7.660(1) \times 10^{37}$ \\
\hline 1495 & 58757.920745782 & $566.1(5)$ & $5.8(7)$ & 0.12 & $7.71 \pm 0.8$ & 0.0447 ( 1) & $4.787(5) \times 10^{37}$ \\
\hline 1496 & 58757.924153111 & $568.0(3)$ & $5.99(3)$ & 0.4 & $19.6 \pm 0.7$ & $0.1174(0)$ & $1.257(0) \times 10^{38}$ \\
\hline 1497 & 58757.924153160 & $569.7(9)$ & $2.12(5)$ & 0.07 & $18.83 \pm 0.9$ & $0.0399(0)$ & $4.273(4) \times 10^{37}$ \\
\hline 1498 & 58757.924486780 & $564.9(6)$ & $5.55(5)$ & 0.15 & $9.09 \pm 9.1$ & $0.0505(4)$ & $5.401(43) \times 10^{37}$ \\
\hline 1499 & 58757.924486791 & $564.9(6)$ & $4.98(3)$ & 0.15 & $9.35 \pm 3.4$ & $0.0466(1)$ & $4.984(11) \times 10^{37}$ \\
\hline 1500 & 58757.925393280 & $565.3(3)$ & $4.76(4)$ & 0.15 & $10.78 \pm 0.7$ & $0.0513(0)$ & $5.493(3) \times 10^{37}$ \\
\hline 1501 & 58757.925395098 & $565.4(3)$ & $3.19(3)$ & 0.15 & $13.26 \pm 0.9$ & $0.0423(0)$ & $4.528(2) \times 10^{37}$ \\
\hline 1502 & 58757.925467366 & $565.4(3)$ & $9.2(8)$ & 0.05 & $7.72 \pm 0.5$ & $0.0710(0)$ & $7.603(4) \times 10^{37}$ \\
\hline 1503 & 58757.925899193 & $565.5(3)$ & 4.48 ( 3 ) & 0.45 & $18.3 \pm 0.9$ & $0.0820(0)$ & $8.776(2) \times 10^{37}$ \\
\hline 1504 & 58757.925899332 & $565.2(4)$ & $3.53(1)$ & 0.45 & $70.89 \pm 1.0$ & $0.2502(0)$ & $2.679(0) \times 10^{38}$ \\
\hline 1505 & 58757.926223123 & $564.5(3)$ & $4.01(2)$ & 0.25 & $23.34 \pm 0.8$ & $0.0936(0)$ & $1.002(0) \times 10^{38}$ \\
\hline 1506 & 58757.926223155 & $567.1(6)$ & $2.59(2)$ & 0.15 & $7.34 \pm 0.8$ & $0.0190(0)$ & $2.035(1) \times 10^{37}$ \\
\hline
\end{tabular}


Table 1:

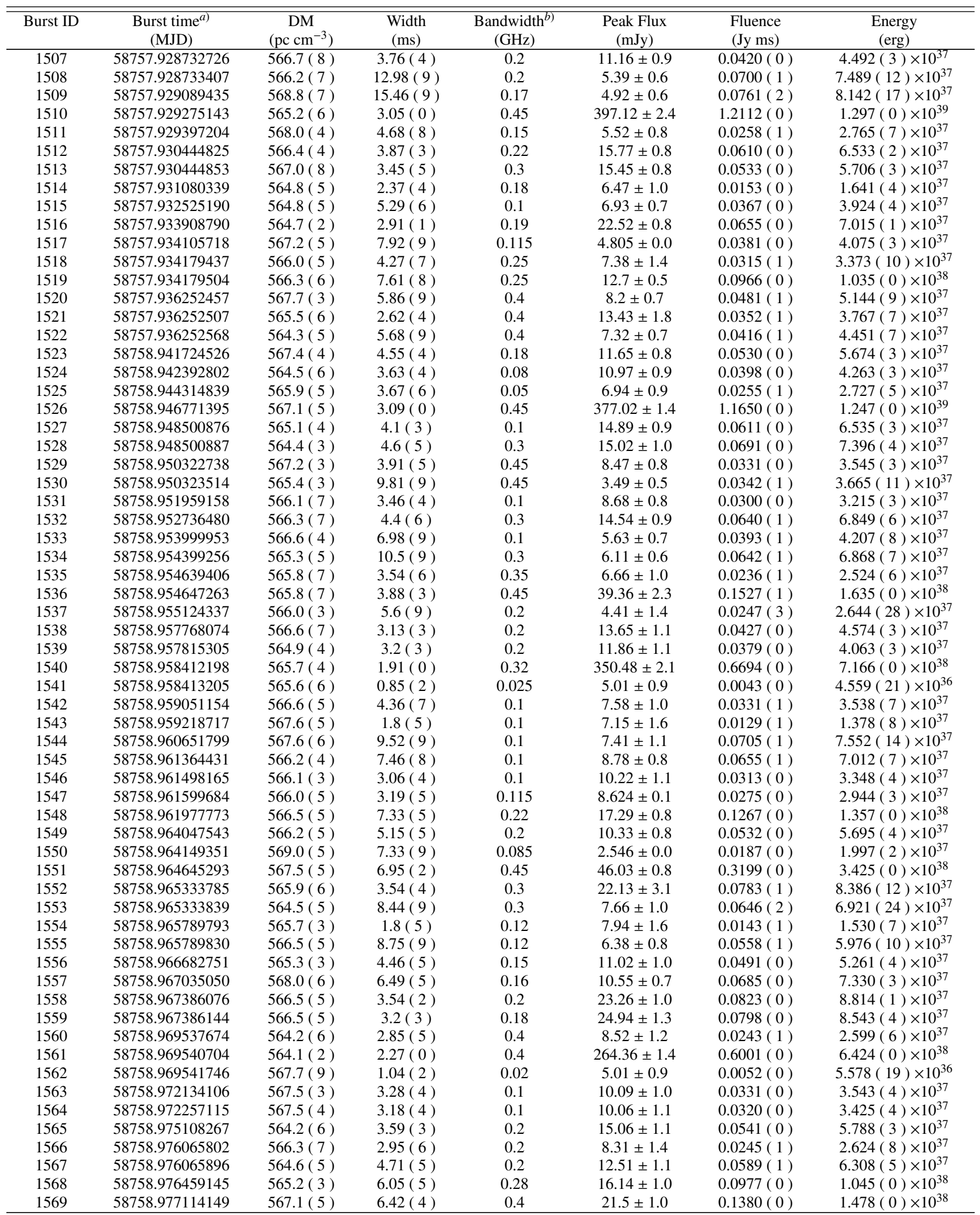


Table 1:

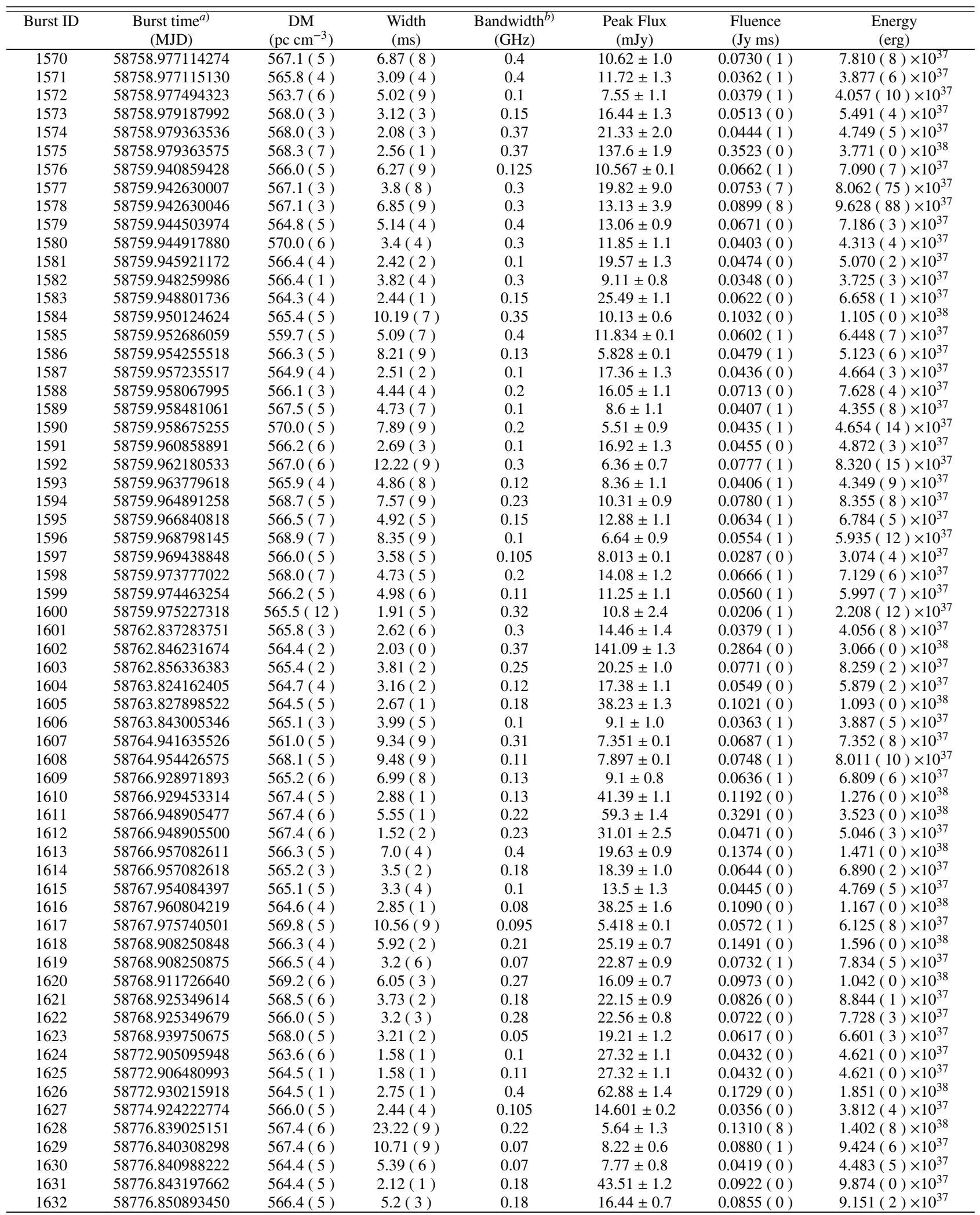


Table 1:

\begin{tabular}{cccccccc}
\hline \hline Burst ID & $\begin{array}{c}\text { Burst time }^{a)} \\
(\mathrm{MJD})\end{array}$ & $\begin{array}{c}\text { DM } \\
\left(\mathrm{pc} \mathrm{cm}^{-3}\right)\end{array}$ & $\begin{array}{c}\text { Width } \\
(\mathrm{ms})\end{array}$ & $\begin{array}{c}\text { Bandwidth }^{b)} \\
(\mathrm{GHz})\end{array}$ & $\begin{array}{c}\text { Peak Flux } \\
(\mathrm{mJy})\end{array}$ & $\begin{array}{c}\text { Fluence } \\
(\mathrm{Jy} \mathrm{ms})\end{array}$ & $\begin{array}{c}\text { Energy } \\
(\mathrm{erg})\end{array}$ \\
\hline 1633 & 58776.850893504 & $569.9(10)$ & $1.48(4)$ & 0.21 & $4.98 \pm 1.2$ & $0.0074(1)$ & $7.890(56) \times 10^{36}$ \\
1634 & 58776.850963341 & $565.7(4)$ & $2.73(1)$ & 0.4 & $45.89 \pm 0.9$ & $0.1253(0)$ & $1.341(0) \times 10^{38}$ \\
1635 & 58776.850963414 & $565.7(11)$ & $6.1(2)$ & 0.4 & $33.46 \pm 0.7$ & $0.2041(0)$ & $2.185(0) \times 10^{38}$ \\
1636 & 58776.855011892 & $565.4(5)$ & $6.46(6)$ & 0.07 & $7.31 \pm 0.6$ & $0.0472(0)$ & $5.055(3) \times 10^{37}$ \\
1637 & 58776.855314910 & $565.2(3)$ & $17.61(9)$ & 0.3 & $3.21 \pm 0.5$ & $0.0565(2)$ & $6.051(21) \times 10^{37}$ \\
1638 & 58776.855315526 & $567.8(6)$ & $9.6(9)$ & 0.4 & $3.43 \pm 0.4$ & $0.0329(1)$ & $3.525(7) \times 10^{37}$ \\
1639 & 58776.855317362 & $567.8(6)$ & $13.99(9)$ & 0.35 & $5.29 \pm 0.4$ & $0.0740(1)$ & $7.922(6) \times 10^{37}$ \\
1640 & 58776.856457273 & $566.0(7)$ & $2.91(1)$ & 0.2 & $38.46 \pm 0.8$ & $0.1119(0)$ & $1.198(0) \times 10^{38}$ \\
1641 & 58776.857352902 & $566.5(6)$ & $3.7(3)$ & 0.2 & $19.3 \pm 2.4$ & $0.0714(1)$ & $7.644(8) \times 10^{37}$ \\
1642 & 58776.857352954 & $566.5(7)$ & $8.37(9)$ & 0.2 & $6.05 \pm 0.8$ & $0.0506(2)$ & $5.421(17) \times 10^{37}$ \\
1643 & 58776.861003441 & $564.7(6)$ & $2.9(4)$ & 0.12 & $7.43 \pm 0.9$ & $0.0215(0)$ & $2.307(4) \times 10^{37}$ \\
1644 & 58776.861252600 & $565.6(5)$ & $1.96(1)$ & 0.4 & $33.88 \pm 1.2$ & $0.0664(0)$ & $7.109(1) \times 10^{37}$ \\
1645 & 58776.868977238 & $566.4(6)$ & $5.38(4)$ & 0.05 & $11.71 \pm 0.7$ & $0.0630(0)$ & $6.744(2) \times 10^{37}$ \\
1646 & 58776.869192493 & $565.4(2)$ & $7.67(9)$ & 0.05 & $6.02 \pm 0.7$ & $0.0462(1)$ & $4.943(7) \times 10^{37}$ \\
1647 & 58776.870213414 & $567.5(5)$ & $3.54(9)$ & 0.32 & $7.01 \pm 3.5$ & $0.0248(4)$ & $2.656(43) \times 10^{37}$ \\
1648 & 58776.870213461 & $567.9(5)$ & $7.67(9)$ & 0.2 & $11.63 \pm 1.2$ & $0.0892(2)$ & $9.549(17) \times 10^{37}$ \\
1649 & 58776.874026246 & $565.5(5)$ & $7.0(5)$ & 0.4 & $10.75 \pm 0.6$ & $0.0752(0)$ & $8.055(3) \times 10^{37}$ \\
1650 & 58776.876920010 & $565.6(5)$ & $2.22(3)$ & 0.16 & $9.33 \pm 1.2$ & $0.0207(0)$ & $2.217(3) \times 10^{37}$ \\
1651 & 58776.877559894 & $567.6(5)$ & $7.98(9)$ & 0.075 & $9.32 \pm 0.1$ & $0.0744(1)$ & $7.965(7) \times 10^{37}$ \\
1652 & 58776.877825786 & $566.0(4)$ & $2.58(4)$ & 0.3 & $46.052 \pm 0.4$ & $0.1190(1)$ & $1.274(1) \times 10^{38}$ \\
& & & & & & & \\
\end{tabular}

\# Uncertainties in parentheses refer to the last quoted digit.

a) Arrival time of burst peak at the solar system barycenter, after correcting to the frequency of $1.5 \mathrm{GHz}$.

b) A conservative $30 \%$ fractional error is assumed. 


\section{Figures}

FRB121102 bursts rate statistics

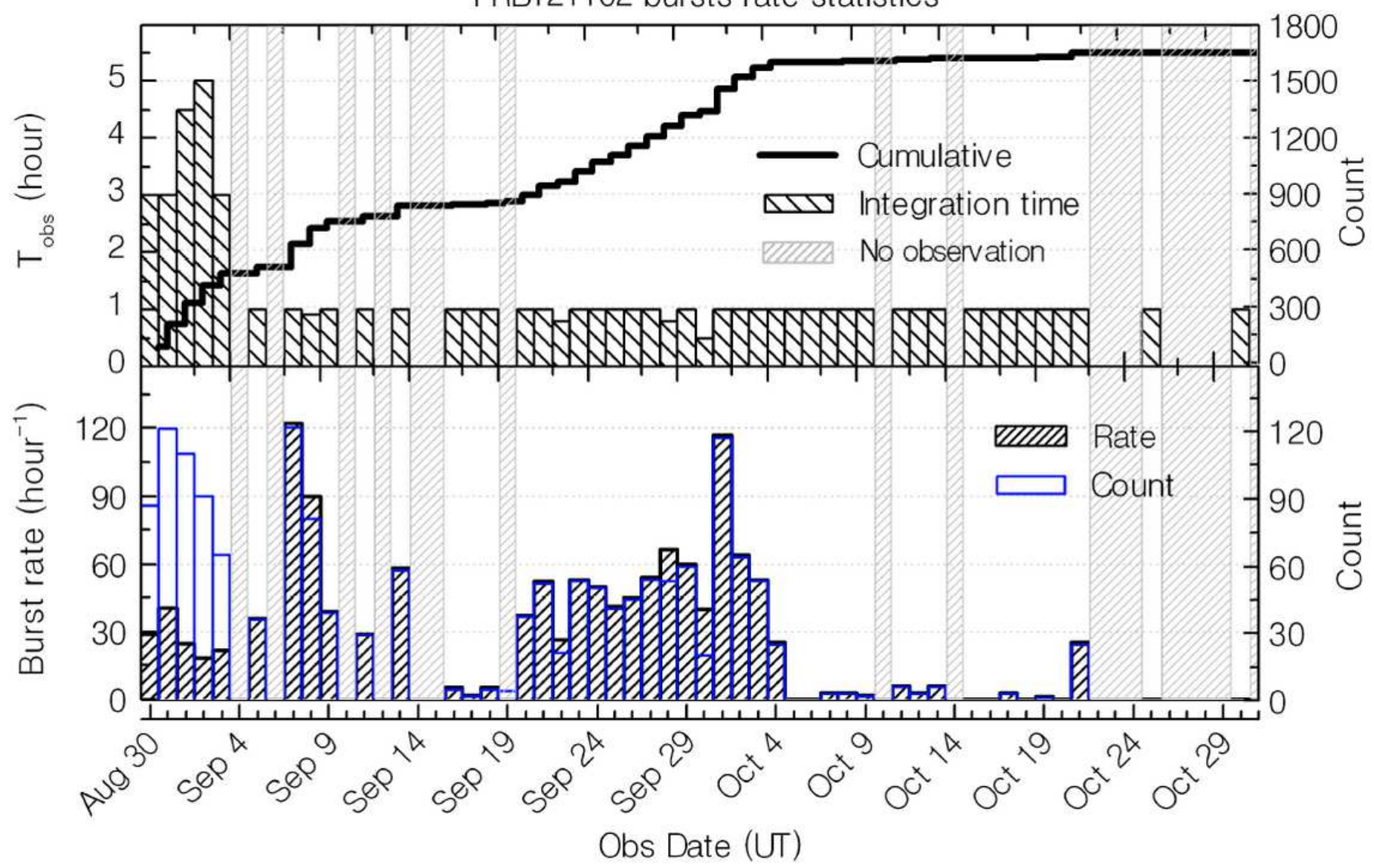

Figure 1

The detected bursts and their distribution during the observing campaign. Upper panel: observing hours and accumulated burst number. Lower panel: burst counts and burst rate. The grey shaded bars denote days without observations of the source. 


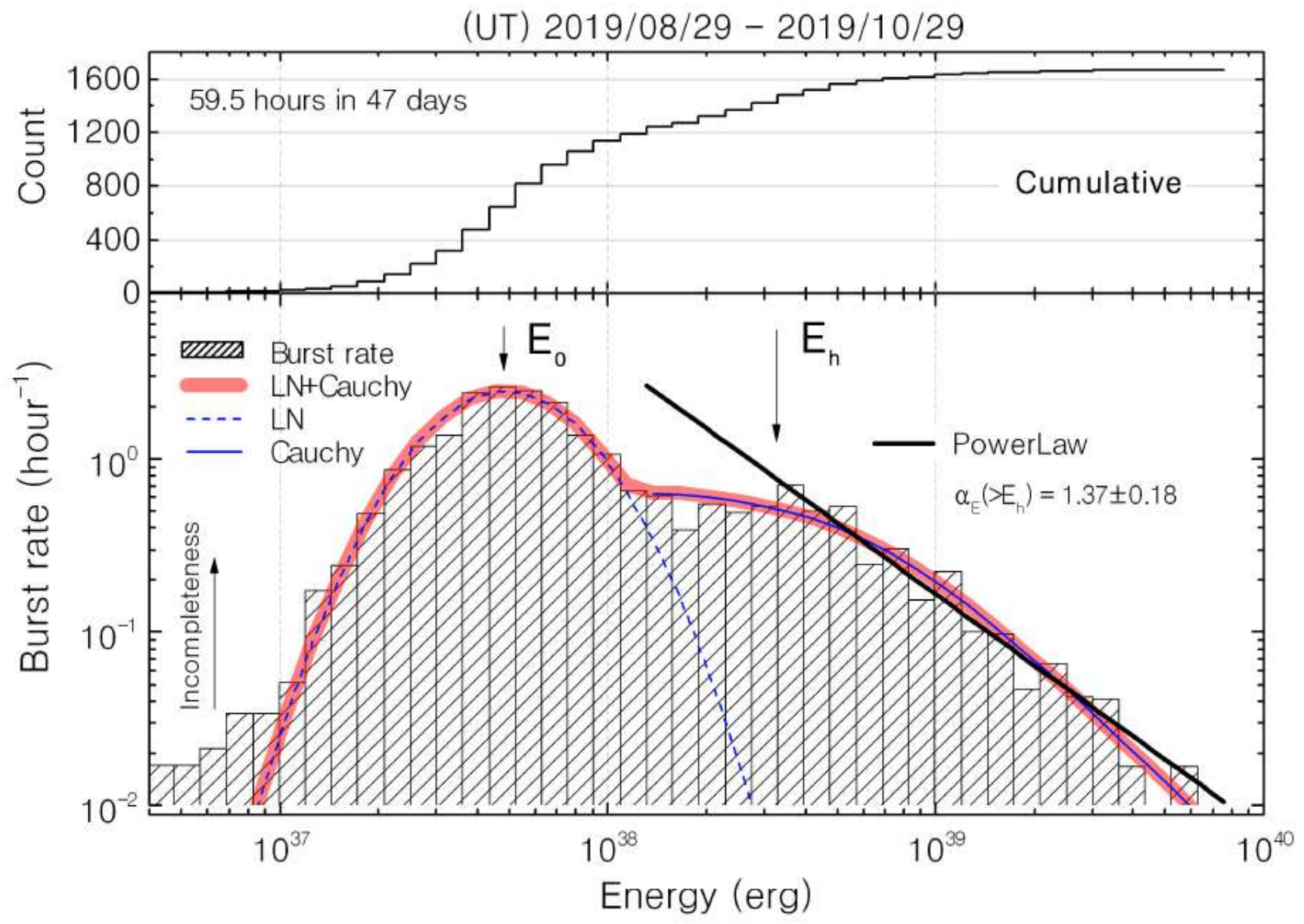

Figure 2

The burst rate distribution of the isotropic equivalent energy at $1.25 \mathrm{GHz}$ for FRB 121102 bursts. The bimodal 'lognormal(LN-dashed blue) + Cauchy (solid blue)' distribution is shown in red and a single power-law fit for bursts above a certain threshold $\mathrm{E} \geq \mathrm{Eh}=3 \times 10^{\wedge} 38 \mathrm{erg}$ is shown in black. Judging by experience, the weak bursts with peak flux smaller $\sim 9 \delta$ cannot be recovered completely (see Methods), as indicated by the upward arrow. 


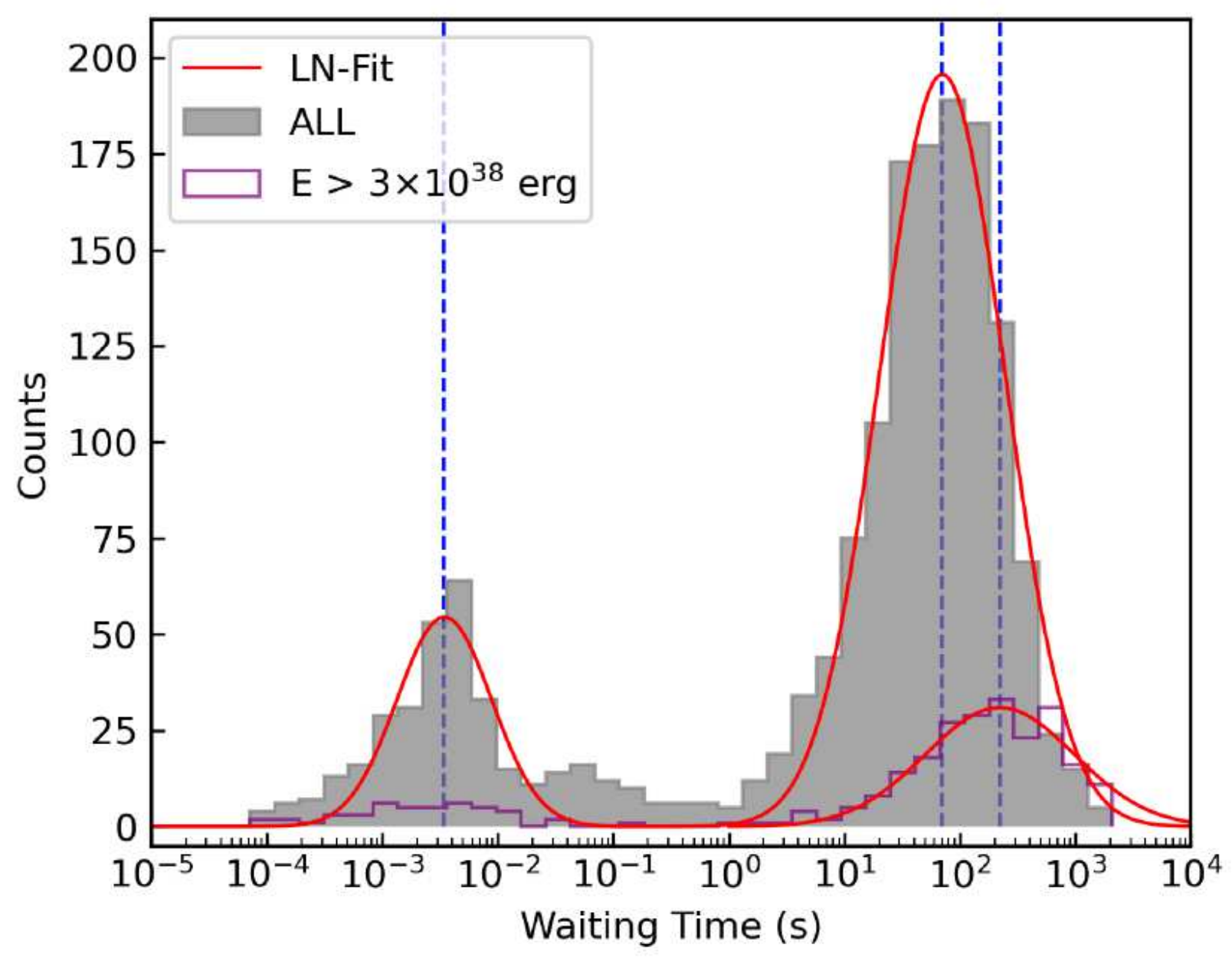

Figure 3

The waiting time distribution of the bursts. The grey bar and solid red curve denote the distribution of waiting time and its log-normal $(L N)$ fit. The high energy component is shown in solid purple line $(E>3$ $x 10^{\wedge} 38 \mathrm{erg}$ ). The three fitted peak waiting times (blue dashed vertical lines) from left to right are $3.4 \square \pm 1.0$ $\mathrm{ms}, 70 \pm 12 \mathrm{~s}$, and $220 \pm 100 \mathrm{~s}$, respectively. (Sedov-Taylor) phase18, 1991 . 

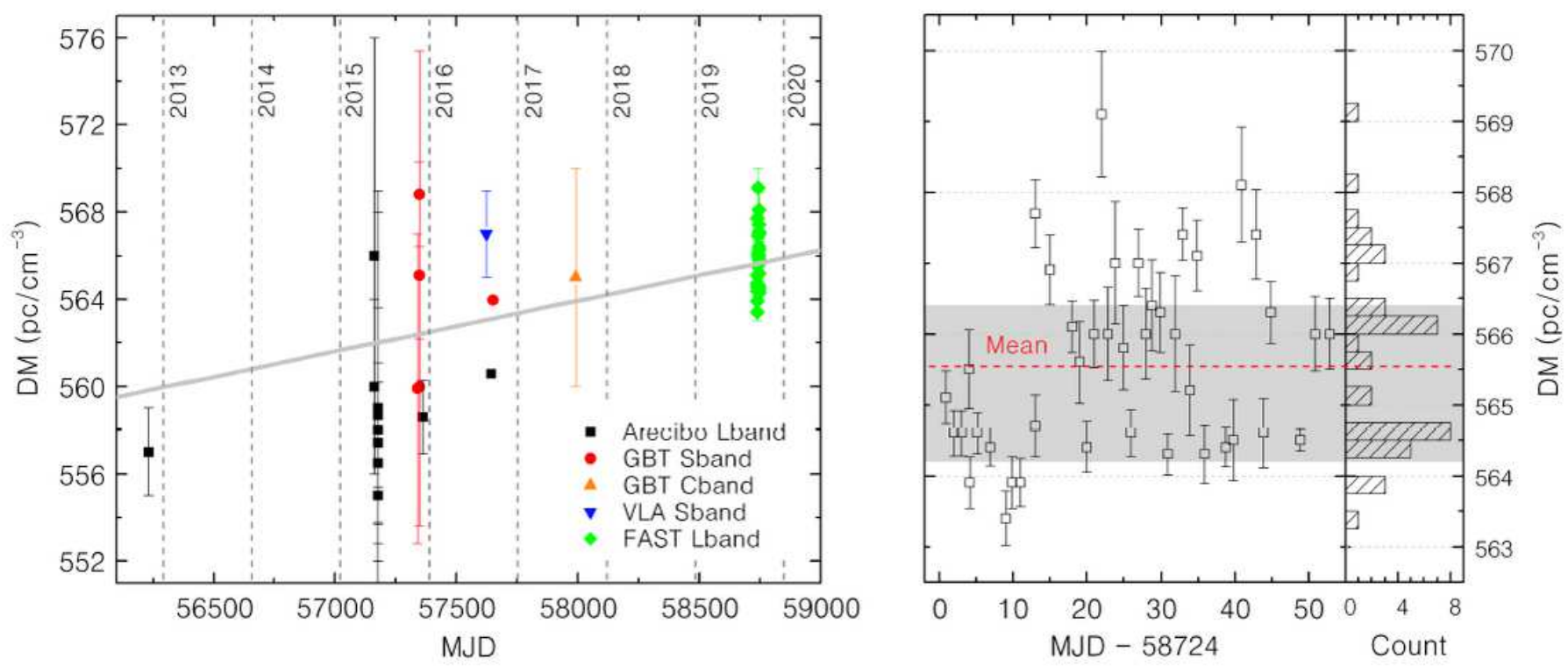

Figure 4

The DM evolution of FRB 121102. Left panel: Temporal DM variation for FRB 121102 over the years. The solid grey line denotes the corresponding linear fitting with a growth rate $+0.85 \pm 0.10 \mathrm{pc} \mathrm{cm}^{\wedge}-3 \mathrm{yr}^{\wedge}-1$. Right panel: the distribution of optimum DM for one of brightest burst in each day during the FAST observations. All the bursts are considered to have a conservative statistical error of FWHM for squared derivative profiles with each DM trial. The red dashed line indicates averaged DM $565.6 \mathrm{pc} \mathrm{cm}^{\wedge}-3$ during the FAST observation, and the grey region shows the $95 \%$ confidence level. 


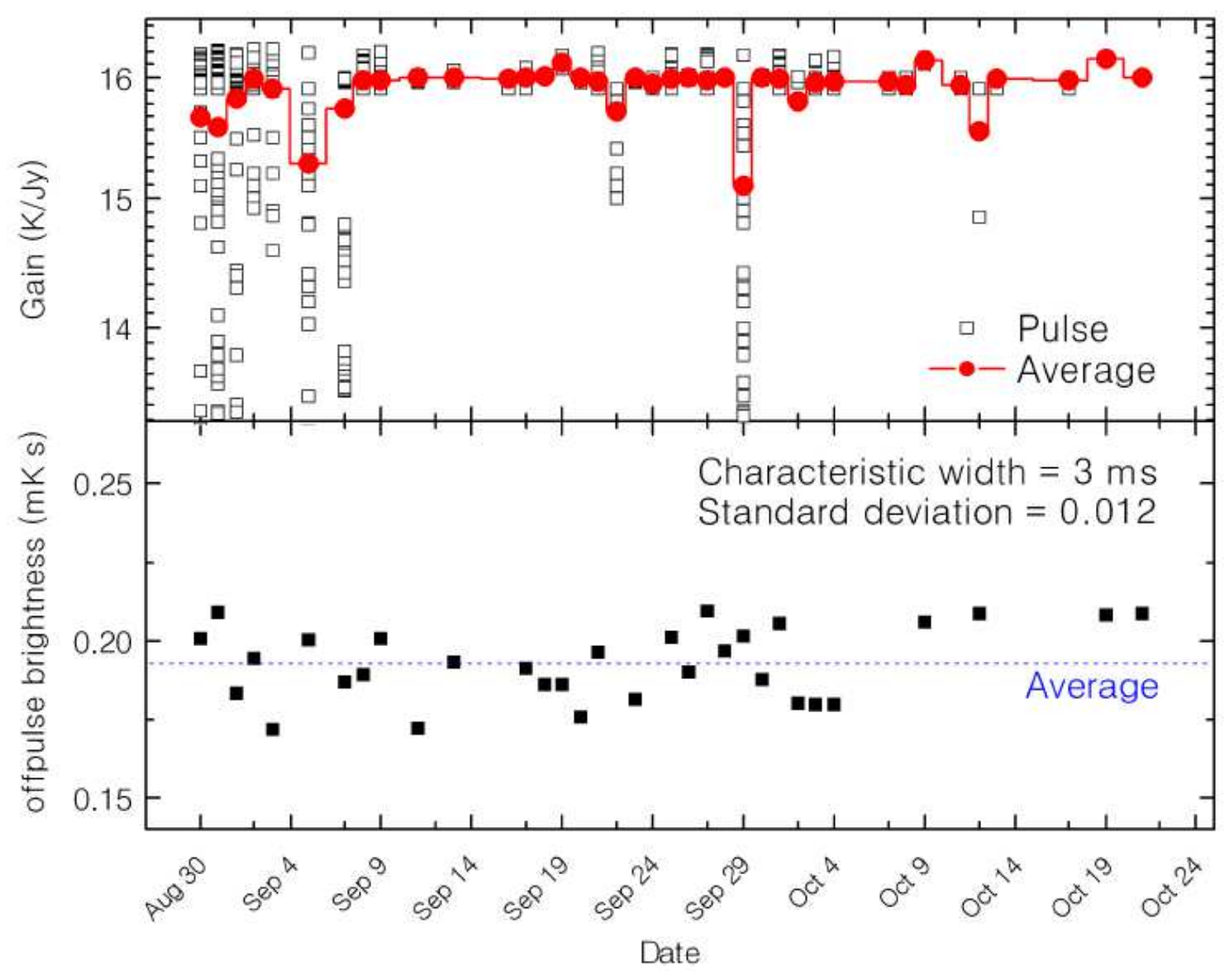

Figure 5

The distribution of the instrumental gain and oه-pulse brightness RMS at $1.25 \mathrm{GHz}$ for observations. The upper panel indicates the gain applied for each pulses. The red dots denote the averaged gain in each day. The bottom panel shows the oख-pulse brightness RMS ( $\mathrm{mK} \mathrm{s}$ ) of first pulse in each day. 


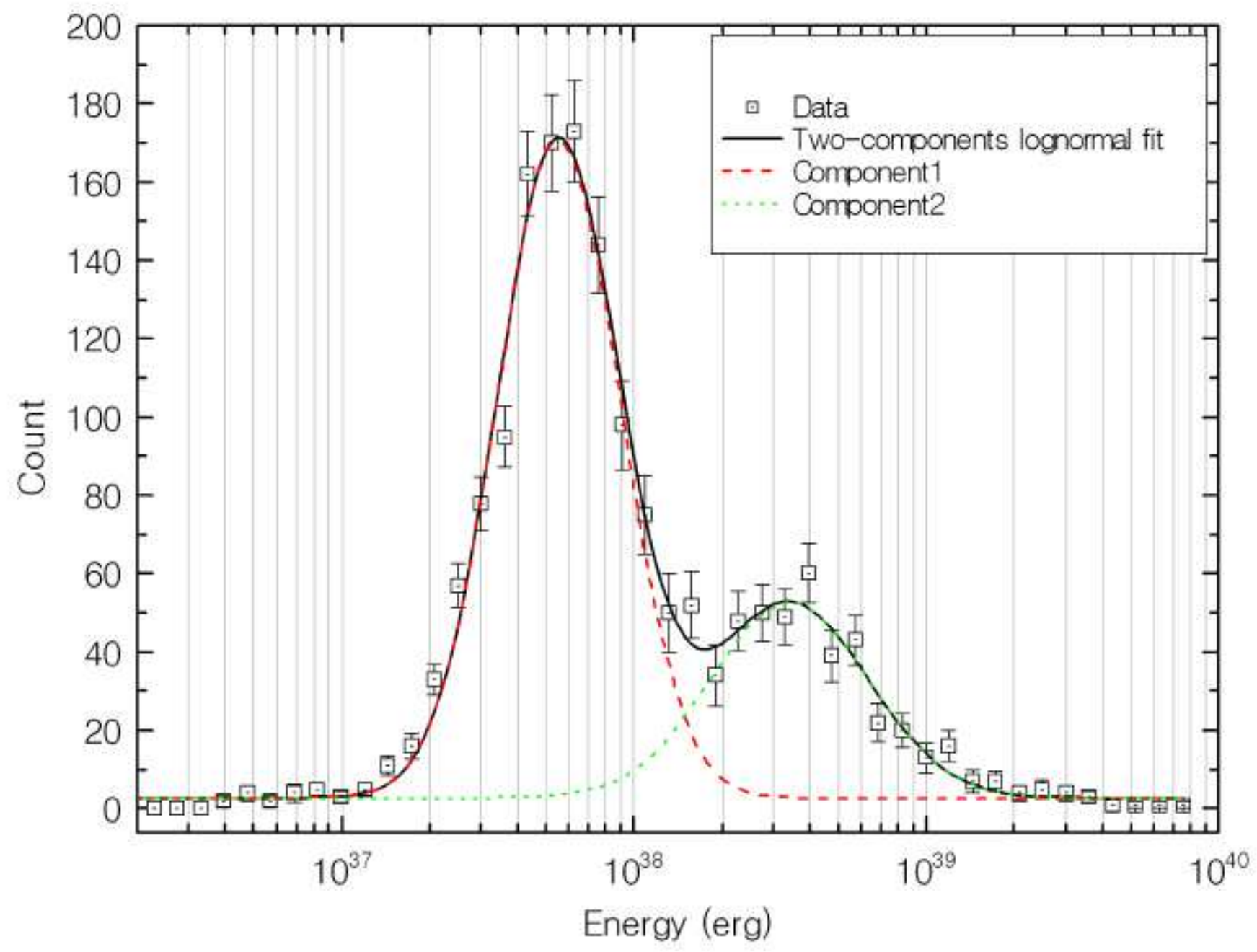

Figure 6

The distribution of the isotropic equivalent energy at $1.25 \mathrm{GHz}$ for FRB 121102 bursts. The twocomponent lognormal distribution is separately fitted in red and green dash lines, an overall fit for bursts is shown in black. 


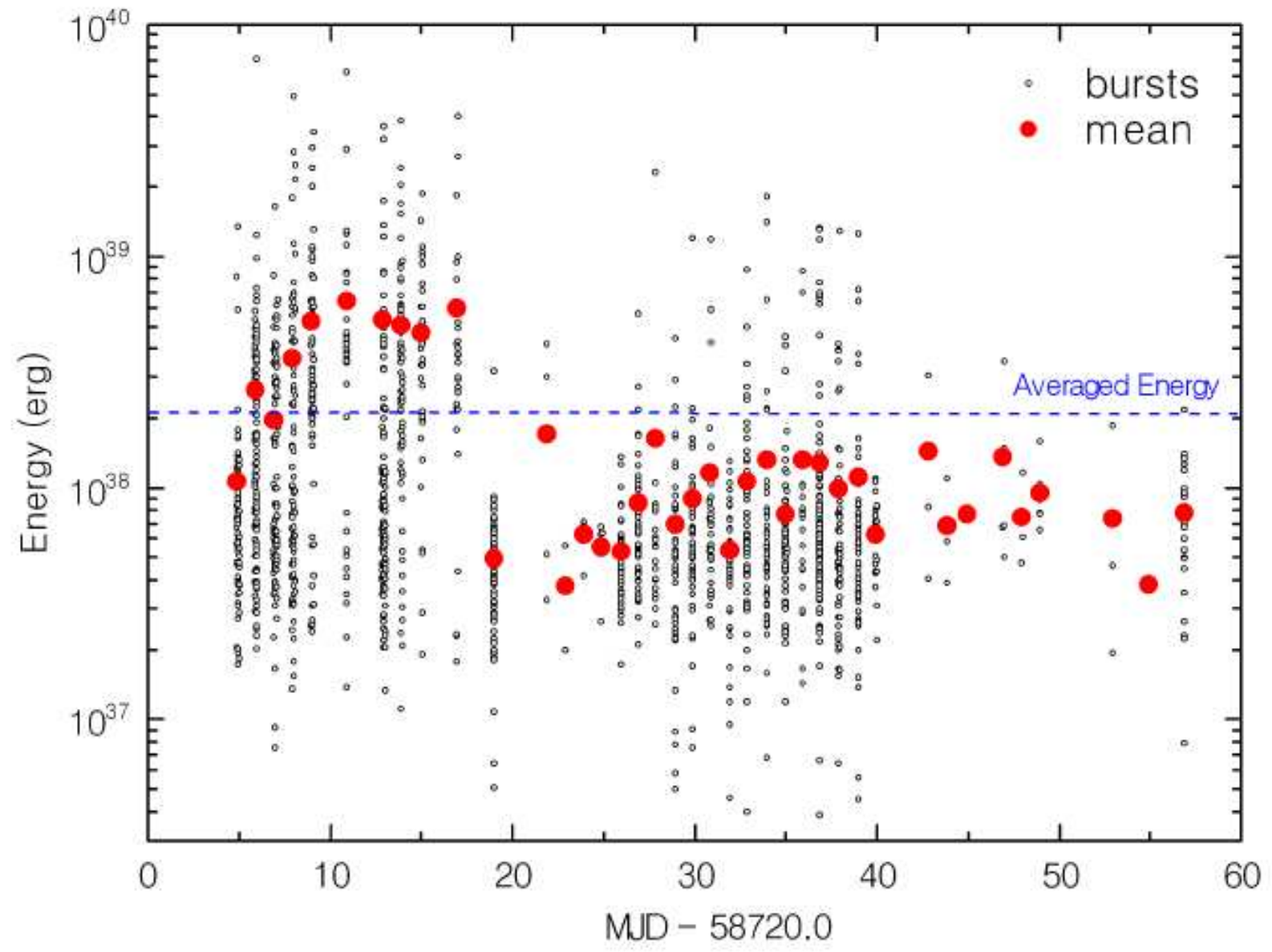

Figure 7

Energy of each burst. The red dots represent the average value for each observing session. An overall averaged is shown as a dashed blue line. 

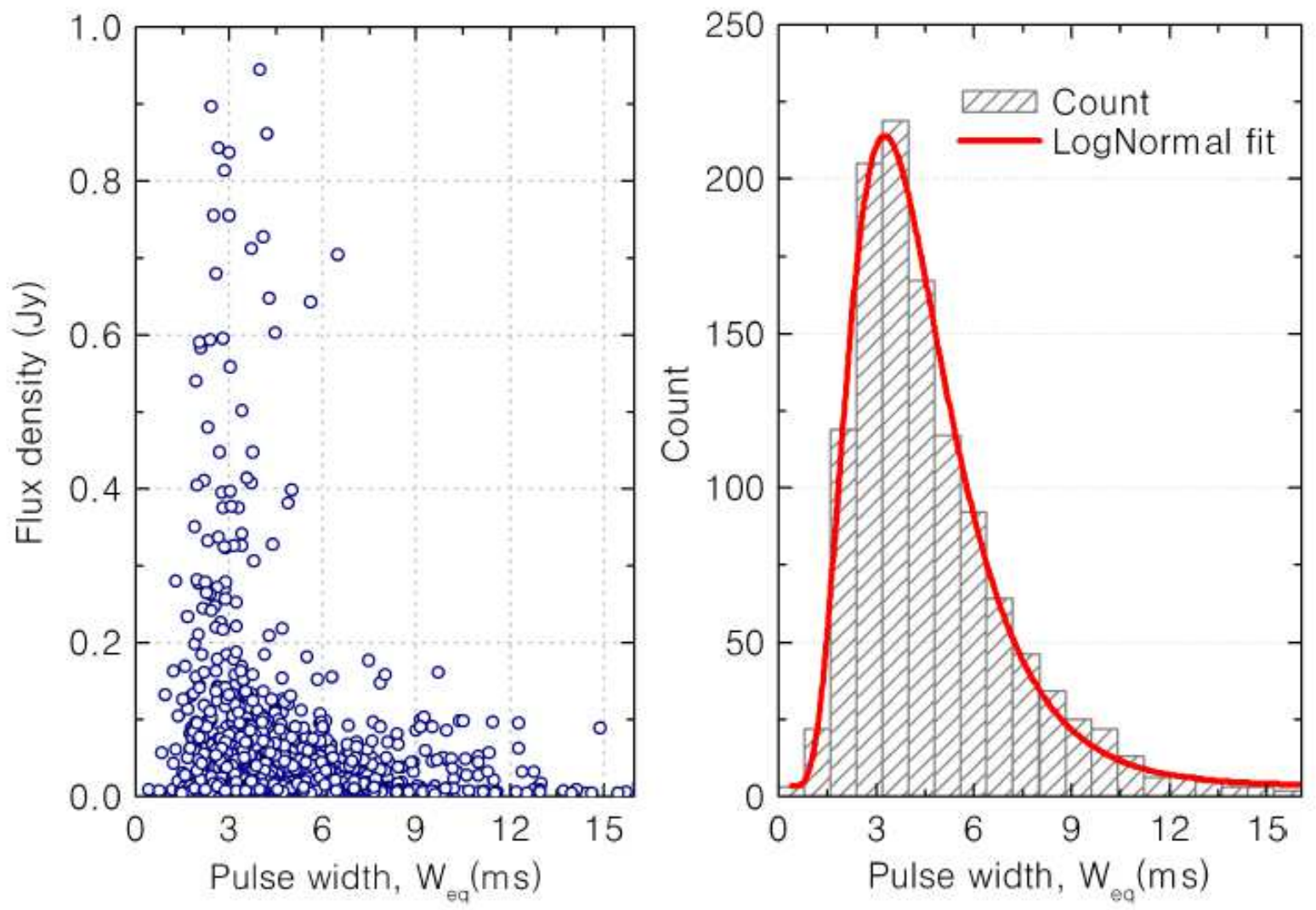

Figure 8

Left: Flux intensity against pulse width for the FRB 121102 bursts in our sample. Right: The equivalent pulse width histogram. 


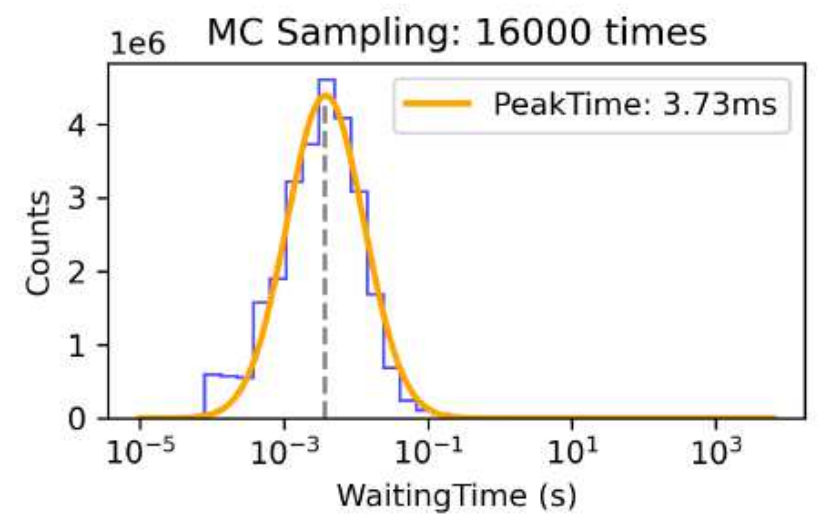

MC Sampling: 1 times

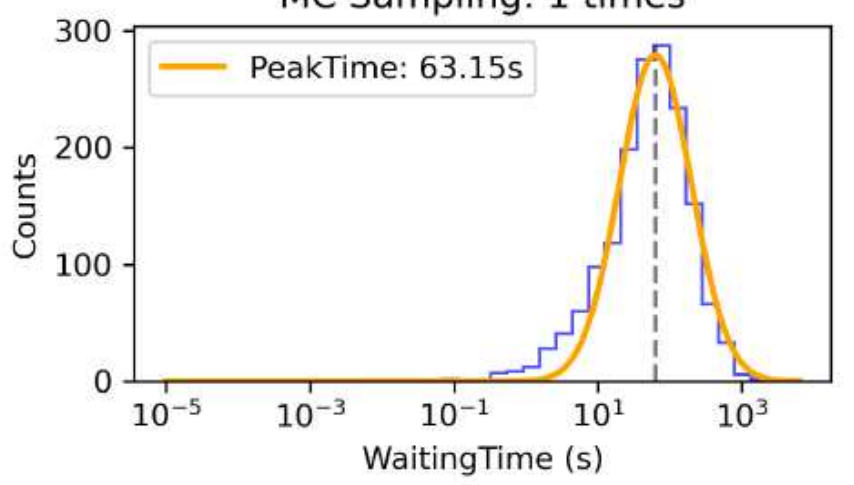

MC Sampling: 100 times

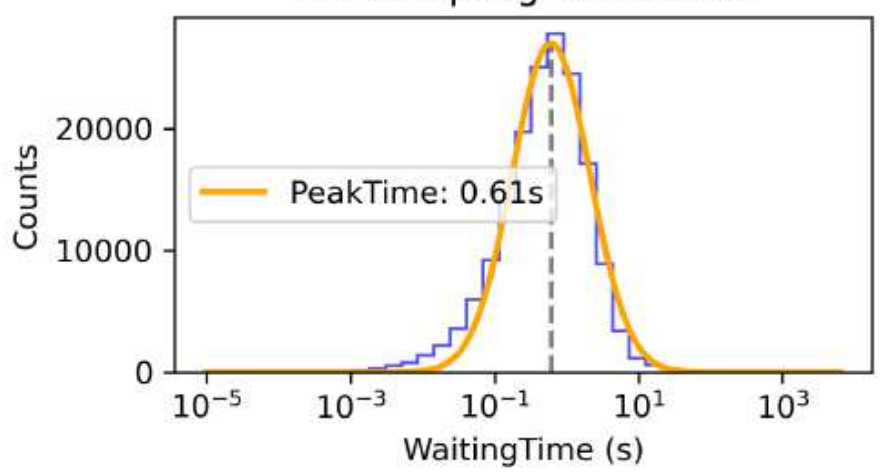

MC Sampling: 0.2 times

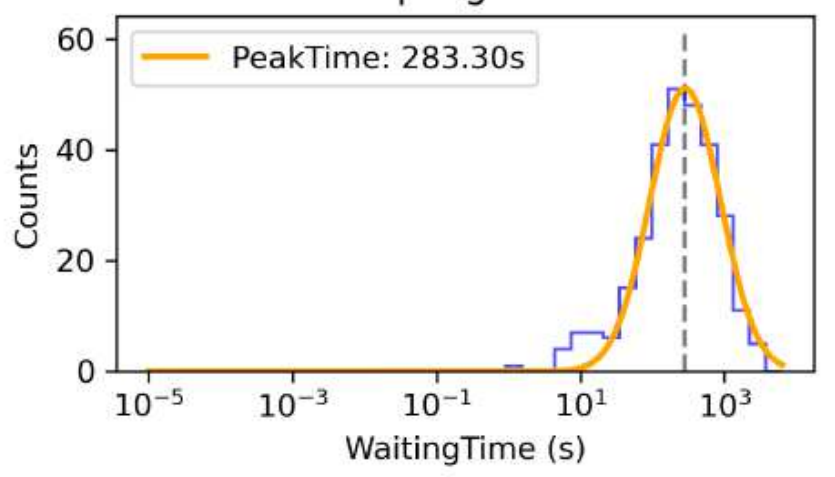

\section{Figure 9}

MC simulations of the waiting time distribution. These four figures show the distribution of waiting time sampled according to 16000 times, 100 times, 1 time, and 0.2 times of the real pulse number. The peak times of the four log-normal distributions are $3.7 \mathrm{~ms}, 0.61 \mathrm{~s}, 63.15 \mathrm{~s}, 283.3 \mathrm{~s}$, respectively.
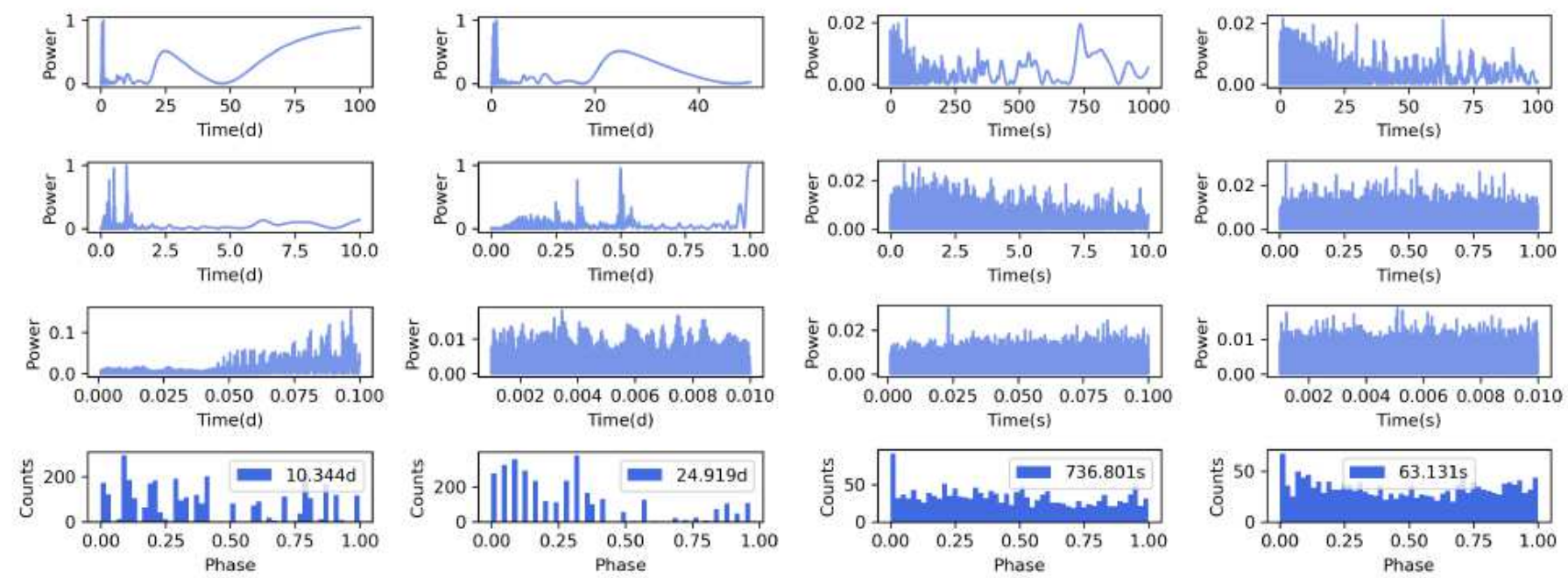
Lomb-Scargle periodogram of FRB121102 burst arrival times. Left: Day periodogram of FRB121102I. Right: Second periodogram of FRB121102.
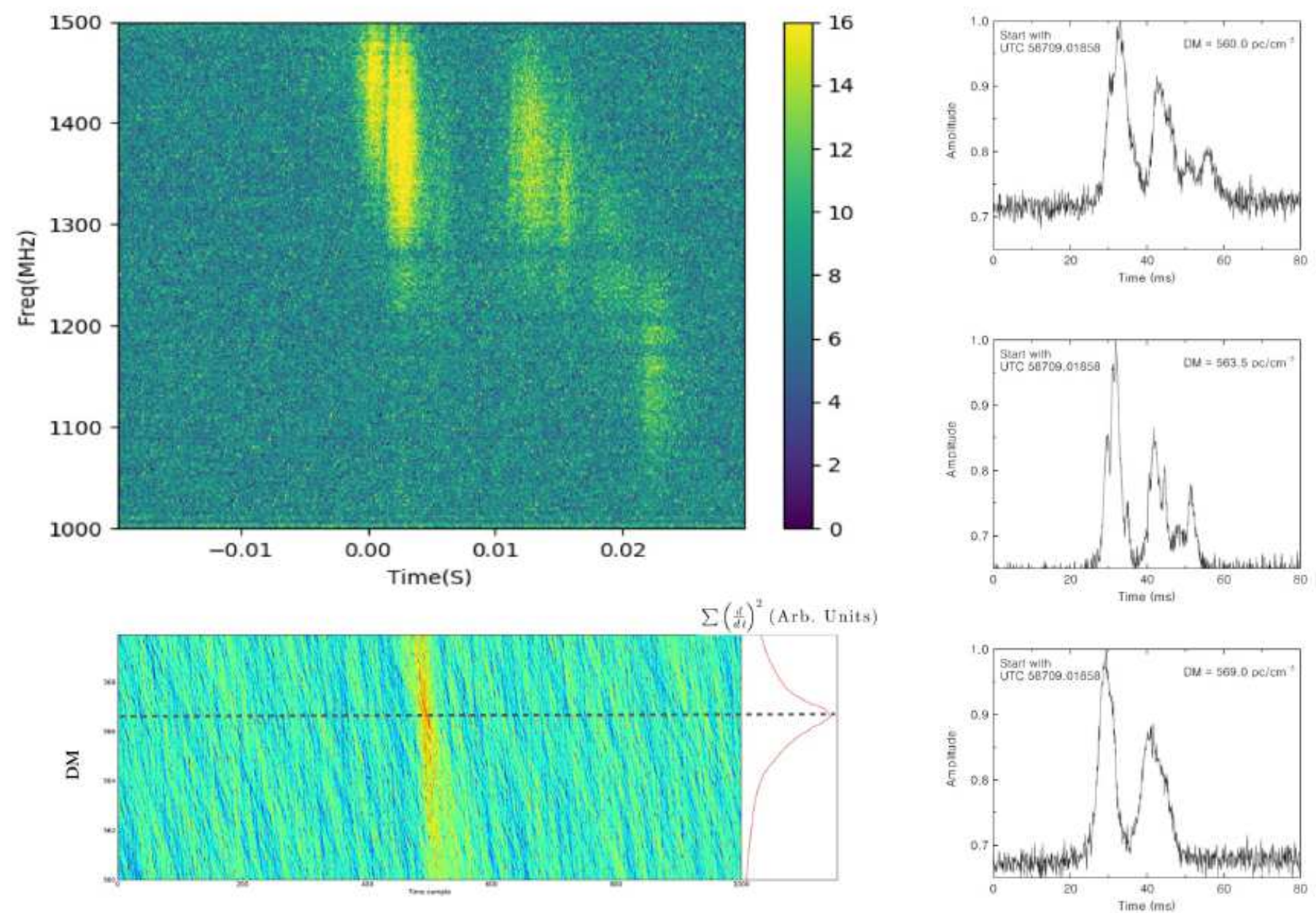

\section{Figure 11}

Example of the DM optimization method for FRB 121102. The complex time-frequency structures for the burst of MJD 58729.01858 was revealed with an optimal DM of $563.5 \mathrm{pc} \mathrm{cm}^{\wedge}-3$. 


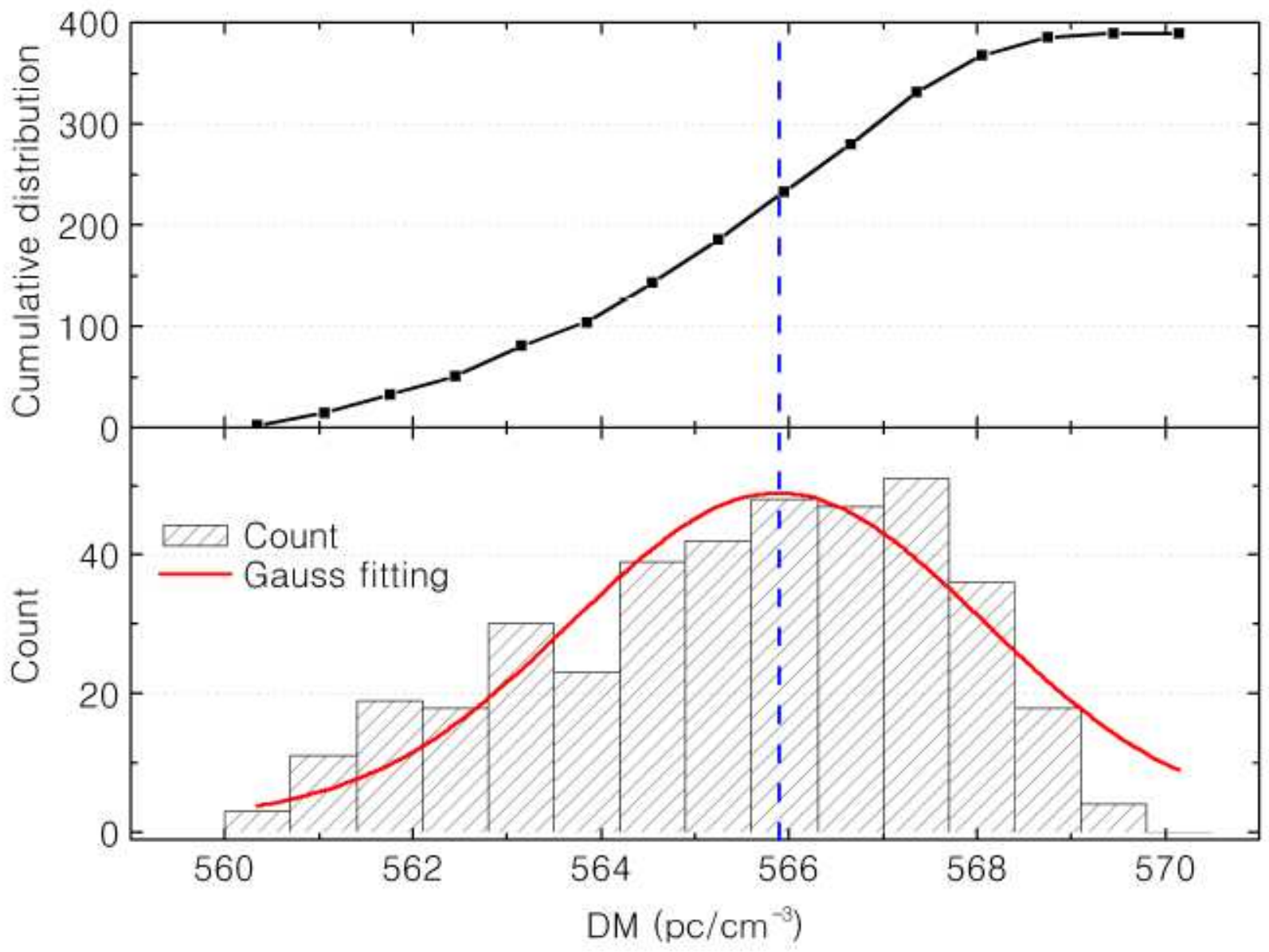

Figure 12

Histogram and cumulative distribution of dispersion measure for FRB 121102. 